\title{
11. MAGNETIC SUSCEPTIBILITY AND PHYSICAL PROPERTIES OF SEDIMENT CORES FROM SITE 893, SANTA BARBARA BASIN: RECORDS OF SEDIMENT DIAGENESIS OR OF PALEOCLIMATIC AND PALEOCEANOGRAPHIC CHANGE?
}

\author{
Frank R. Rack, ${ }^{2}$ Elizabeth A. Heise, ${ }^{3}$ and Ruediger Stein ${ }^{4}$
}

\begin{abstract}
Magnetic susceptibility measurements were made on whole-core, split-core, and discrete samples from Ocean Drilling Program Site 893, located in the central Santa Barbara Basin. These measurements are presented and preliminary evaluations are made regarding the relationships between magnetic susceptibility, index properties (density, porosity, and water content), and geochemical variables (weight percentage of calcium carbonate and total organic carbon) over the past 160,000 yr.

"Gray beds" in the sediment column, which are faithfully identified by magnetic susceptibility profiles, are most abundant and best preserved within Holocene intervals of laminated sediments and during oxygen isotope Stage 5. The preservation of these gray beds is associated with intervals of lowered sea level and increased global ice mass, as determined from oxygen isotopes.

Calculations of the magnetic susceptibility flux for Hole 893A have been made using an age model based on both accelerator mass spectrometer ${ }^{14} \mathrm{C}$ age dates and the oxygen isotope stratigraphy presented in this volume. The magnetic susceptibility flux may provide a proxy indicator of the relative intensity of terrigenous input into the Santa Barbara Basin and/or may reflect changes in the geochemical environment of the basin through time.

Preliminary observations suggest that magnetic susceptibility measurements may be sensitive to changes in the weight percentage of sedimentary components (e.g., organic matter and to a lesser extent calcium carbonate). It is not yet clear whether this is caused solely by dilution effects or whether this indicates magnetite dissolution during sediment diagenesis.

Finally, we evaluate the Site 893 data using available (regional and global) paleoclimatic records and the results of Holocene modeling runs, in order to assess the possible significance of the susceptibility data as a proxy recorder of paleoceanographic and/or paleoclimatic processes along the western margin of North America.
\end{abstract}

\section{INTRODUCTION}

Magnetic susceptibility measurements were made on whole-core, split-core, and discrete samples from Ocean Drilling Program (ODP) Site 893, located in the central Santa Barbara Basin. These measurements are used to make a preliminary assessment of the relationships between magnetic susceptibility, index properties (density, porosity, and water content), and geochemical variables (weight percentage of calcium carbonate and organic matter) at this site,

Preliminary observations suggest that magnetic susceptibility measurements at Site 893 may be sensitive to changes in the weight percentage of sedimentary components (e.g., organic matter and to a lesser extent calcium carbonate). On the basis of a relatively small number of samples (53), we show that magnetic susceptibility values decrease as the weight percentage of calcium carbonate or total organic carbon increase. It is not yet clear whether this is primarily caused by dilution effects or sediment diagenesis.

The magnetic susceptibility and digital color profiles faithfully record the presence of "gray beds" in laminated intervals of the sediment column, although relatively thin beds $(<1 \mathrm{~cm}$ thick) may not be detected using the susceptibility method because of the relatively

'Kennett, J.P.. Baldauf, J.G., and Lyle, M. (Eds.), 1995. Proc. ODP, Sci. Results. 146 (Pt. 2): College Station. TX (Ocean Drilling Program).

Ocean Mapping Group, Department of Geodesy and Geomatics Engineering, University of New Brunswick, Fredericton, New Brunswick, E3B 5A3, Canada.

Department of Geology and Geophysics, Texas A\&M University, College Station. TX 77843, U.S.A.

${ }^{4}$ Alfred-Wegener-Institute for Polar and Marine Research, Columbusstrasse, D. 27568 Bremerhaven. Federal Republic of Germany. weak signal and because of the specific sensor characteristics (each measurement is averaged over a few centimeters by the Bartington susceptibility loop).

Rack and Merrill (this volume) have convincingly demonstrated that distinctive features, which are identified as peaks and troughs in both magnetic susceptibility and digital color data and often correspond to gray beds, massive intervals, and sand layers in the cores, can be used to correlate between Holes 893A and 893B; they also show that these correlations can be used to transfer the Hole $893 \mathrm{~A}$ age model to Hole $893 \mathrm{~B}$. The study by Rack and Merrill was restricted to the upper 24 mbsf of sediment in each hole (i.e., 0-16 k.y.). We use the same procedures in this paper to correlate magnetic susceptibility peaks in the deeper (older) portions of Holes 893A and 893B.

In order to examine the relative magnitude of the susceptibility signal and to assess differences in the rates of accumulation of magnetic minerals at this site, we have calculated the magnetic susceptibility flux for each sample interval; this technique was previously used in studies of Chinese loess (Maher and Thompson, 1991) and in studies of the distribution of "Heinrich layers" in the North Atlantic Ocean (Grousset et al., 1993). These calculations of the magnetic susceptibility flux may provide a useful proxy indicator of changes in the relative intensity of terrigenous input into the central region of the Santa Barbara Basin; alternatively, variations in the susceptibility flux may reflect changes in various geochemical processes acting on these sediments through time (e.g., during glacial vs. interglacial periods). In the final section of this paper, we present a discussion of some comparisons that were made between the magnetic susceptibility data from Hole 893A and other paleoclimatic records. We do this in order to assess the possible sensitivity of the Site 893 magnetic susceptibility flux record as a proxy recorder of paleoceanographic pro- 

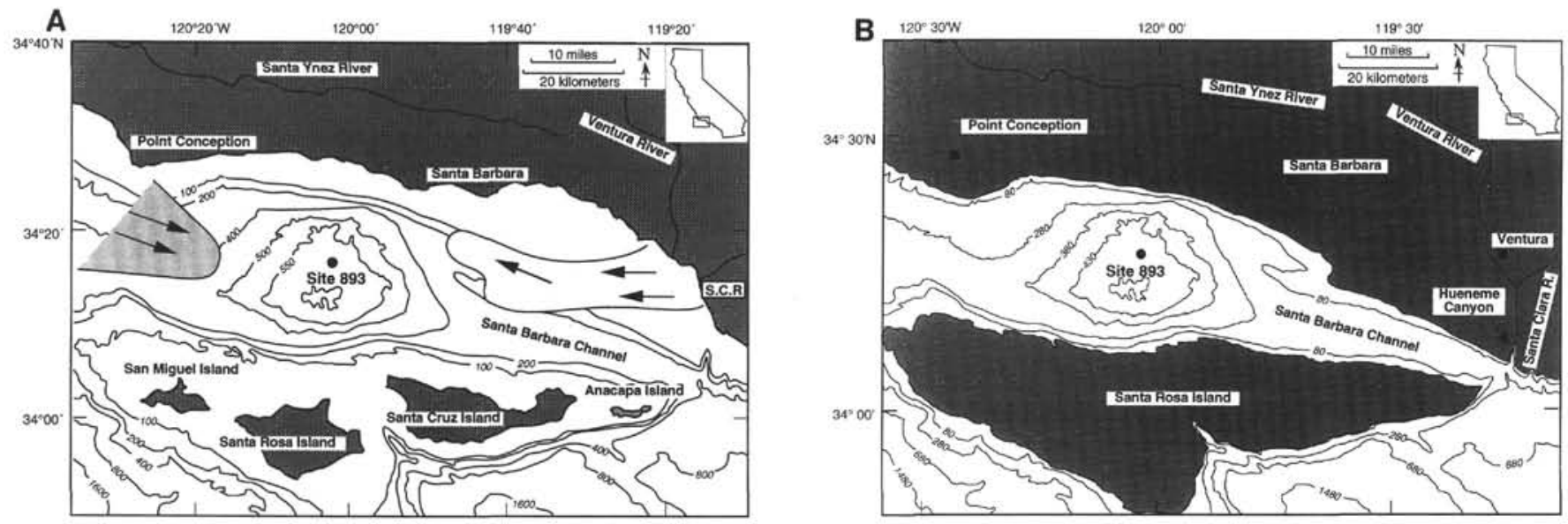

Figure 1. A. Bathymetric map of the Santa Barbara Basin showing the location of Site 893 (modified from Kennett, Baldauf, et al., 1994). Sources of river derived silt (from the east-Santa Clara and Ventura Rivers) and fan-derived silt (from the west of Point Conception) are also shown (after Thornton, 1984). B. Estimated bathymetry of the Santa Barbara Basin during the Last Glacial Maximum (18,000 yr ago) showing the location of Site 893 (modified from Kennett, Baldauf, et al., 1994). Note the expansion of land areas seaward and the resulting restriction of the Santa Barbara Channel to the southeast.

cesses and/or Quaternary paleoclimatic changes over western North America.

We compare the records from Hole $893 \mathrm{~A}$ with estimates of ice mass deduced from the SPECMAP $\delta^{18} \mathrm{O}$ data set (Imbrie et al., 1984; Saltzman and Verbitsky, 1994), which provide a relative indicator of the rate of change and magnitude of variations in global mean sea level. We also present a graphical comparison between our results and the Greenland Ice-core Project (GRIP) oxygen isotope record (GRIP Members, 1993). We do this to assess the possible relationships between the magnetic susceptibility fluctuations in Hole 893A and changing atmospheric circulation patterns (i.e., regional, continental scale, and/or global patterns).

We propose that changes in terrigenous flux to the Santa Barbara Basin are indicative of increased rainfall and subsequent runoff from coastal regions during glacial and interglacial periods. These inferred climatic changes across western North America were likely caused by shifts in atmospheric circulation patterns which resulted in regional changes in the amounts of precipitation minus evaporation (Cooperative Holocene Mapping Project [COHMAP] Members, 1988; Thompson et al., 1993).

We also speculate about whether the timing and amplitude of transient shifts in oxygen isotope values from the GRIP ice core from Summit, Greenland (Dansgaard et al., 1993), which may reflect atmospheric circulation changes (GRIP Members, 1993) and/or changes in the amount of precipitation reaching Greenland from western (Pacific) source regions (Charles et al., 1994), show a similar pattern of fluctuations as we observe in the susceptibility record from Site 893

\section{BACKGROUND AND OBJECTIVES} Site 893

Site 893 is located at $34^{\circ} 17.25 \mathrm{~N}, 120^{\circ} 02.2 \mathrm{~W}$, on the floor of the Santa Barbara Basin (SBB), California, $20 \mathrm{~km}$ south of the Santa Barbara coastline at a water depth of $576.5 \mathrm{~m}$ (Kennett, Baldauf, et al., 1994; Fig. 1A). Two holes were drilled at Site 893; Hole 893A recovered a total of 21 advanced hydraulic piston cores (APC) to a depth of $196.5 \mathrm{~m}$ below the seafloor (mbsf), while Hole 893B recovered a total of eight APC cores to a depth of $68.8 \mathrm{mbsf}$. The sediments recovered at this site are generally a mixture of terrigenous (clays, silty clays, and sands) and biogenic (carbonate and opal) material. Laminated and nonlaminated (massive and/or bioturbated) beds are ob- served throughout, both as interlayered features and as dominant structural styles within specific zones of the sedimentary sequence recovered at Site 893 (Kennett, Baldauf, et al., 1994).

The Santa Barbara Basin has a bathymetric sill located at either end; the deepest sill lies to the west of Site 893 at a depth of 475 m; a shallower sill is located on the eastern boundary of the basin in the Santa Barbara Channel between the California coast and the Anacapa Islands. To the east of Site 893 lies the Santa Barbara-Oxnard Shelf, a northeast-southwest-trending topographic feature of about $40 \mathrm{~km}$ in length with approximately $800 \mathrm{~km}^{2}$ of total area shallower than 100 $\mathrm{m}$ (Drake et al., 1972). There are several structurally controlled topographic features on the shelf (e.g., Santa Clara [Montalvo] Ridge and Santa Clara [Montalvo] Trough) that influence the sedimentologic regime and the along-shelf transport of sediment in the basin (Drake et al., 1972; Thornton, 1984; Kolpack and Drake, 1985).

The Santa Clara and Ventura rivers drain into the Santa Barbara Channel along its eastern boundary. Much of the sediment from these rivers is sorted by nearshore wave action and then deposited elsewhere in the basin, generally to the northwest; although some of the sediment (especially the coarse sand fraction) may be transported to the southeast by longshore drift and funneled into the Hueneme and Mugu canyons (Reynolds, 1987).

Several authors have estimated the relative contributions of different sediment sources to the Santa Barbara Basin (Fleischer, 1972) and to other California borderland basins (Brownlie and Taylor, 1981; Schwalbach and Gorsline, 1985). Fleischer (1972), who based his estimates on calculations of geographic drainage areas, provides the following breakdown of relative source contributions: Santa Clara River, $51 \%$; Santa Ynez River, 27\%; coastal drainage, 12\%; Ventura River, $7 \%$; and island drainage, $3 \%$. This breakdown highlights the importance of sedimentary input to the eastern basin from the Santa Clara and Ventura rivers (58\% combined).

The major source of sediment to the western basin is from the Santa Ynez River, which empties into the Pacific Ocean north of Point Arguello. Sediments from the Santa Ynez River are transported southeastward by currents flowing around Point Conception and into the western end of the Santa Barbara Basin, where a fan-shaped sedimentary feature is observed (Kolpack, 1986). The relative contributions of sediment from these sources have most likely been different during glacial times, when the geographic boundaries of the basin were more restricted because of falling sea levels (Fig. 1B) and when there were surely many changes in climatic variables (COHMAP Members, 1988) that may have influenced the rate of precipitation 
and the subsequent rates of continental runoff into the Santa Barbara Basin.

\section{Gray Beds}

Gray beds of terrigenous silty clay are frequently observed within the laminated intervals of Site 893 but are less often preserved in the bioturbated or massive zones. These gray silty beds were the subject of several previous studies (Fleischer, 1972; Thornton, 1984; among others) where they are described as "suspended-load deposits derived mainly from the Santa Clara River during large floods" (Fleischer, 1972). Alternatively, some of the gray silty beds were described as fine-grained turbidites, possibly originating from a "line source" (laterally distributed region of slope failure) on the upper shelf region to the west of Santa Barbara (Thornton, 1984); essentially to the north or northeast of Site 893.

The proposed origin of these gray beds, as resulting from flood events, was extensively investigated following an extreme flooding event in 1969. During 1969, flood waters from the Santa Clara and Ventura Rivers transported more than $45-50 \times 10^{6}$ metric tons of terrigenous sediment to the shelf region of the SBB (Brownlie and Taylor, 1981). Much of this fine-grained sediment was later eroded, resuspended, transported to the northwest and basinward by currents along the outer shelf and slope, and ultimately deposited in the deeper basin (Drake et al., 1972; Kolpack and Drake, 1985). Brownlie and Taylor (1981) estimated that the average annual sediment yield of the Santa Clara River between 1928 and 1975 was $3.5 \times 10^{6}$ metric tons of sediment $/ \mathrm{yr}$; over the $47-\mathrm{yr}$ period investigated, almost $55 \%$ of the total sediment yield from this river occurred during the two flood years of 1941 and 1969 (Brownlie and Taylor, 1981).

These observations suggest that the intermittent input of large sediment volumes to the SBB (runoff "events") is a significant feature of the sedimentary budget of this basin and one reasonable explanation for the presence of gray beds. The thickness of gray beds, corrected for compaction effects, may provide an improved understanding of the magnitude of these runoff events, as suggested by Fleischer (1972).

\section{Laminated Sediments}

The occurrence of laminated intervals in the sediment of the Santa Barbara Basin is thought to be primarily controlled by the oxygenation of bottom waters and the presence of bacterial mats on the seafloor, where the lack of bioturbation by organisms and the resulting preservation of laminations occurs when oxygen levels in the bottom water decrease below some critical value (Emery and Hülsemann, 1962; Fleischer, 1972; Soutar and Crill, 1977; Reimers et al., 1990). Recent studies have focused on changes in specific geochemical relationships and on the cycling of elements (e.g., organic carbon, nitrogen sulfur, and oxygen; Berelson et al., 1987; Jahnke, 1990; Schimmelman and Kastner, 1993) in borderland basins, as factors which influence the preservation of sedimentologic events and paleoclimatic signals in these records (Lange et al., 1987, 1990; Schimmelman et al., 1990, 1992).

\section{Magnetic Susceptibility}

Increasing numbers of paleoceanographic studies are using rock magnetic measurements to determine physical changes in marine sedimentary environments or as proxy records of paleoceanographic processes (Bloemendal et al., 1988, 1992, 1993; Bloemendal and deMenocal, 1989; deMenocal et al., 1991; Doh et al., 1988; Grousset et al., 1993; Robinson, 1986; among many others). In anoxic or suboxic basins, and in shelf regions where a distinct oxygen-minimum zone develops, it was convincingly demonstrated that certain geochemical processes are fundamentally important to the preservation and subse- quent interpretation of sediment magnetic records (Karlin and Levi, $1983,1985)$. In these settings, it is often necessary to determine the effects of magnetite dissolution and iron-sulfur diagenesis on the observed paleomagnetic record (Karlin, 1990a, 1990b; Leslie et al., $1990 \mathrm{a}, 1990 \mathrm{~b})$, so that it is possible to identify which of the observed changes are related to paleoceanographic fluctuations and which are caused by diagenetic overprints (Lund et al., 1992).

The topic of a number of recent published papers has been the rock magnetic properties of sediments from several California borderland basins (Brandsma et al., 1989; Leslie et al., 1990a, 1990b; Lund et al., 1992; among others). These studies, which were primarily based on piston and box cores, have shown that distinct differences in sedimentation rate, composition, and magnetic properties can be detected between inner borderland basins (e.g., Santa Barbara, Santa Monica, and San Pedro basins), central borderland basins (e.g., Santa Cruz, Santa Catalina, and San Nicolas basins), and outer borderland basins (e.g., Tanner Basin). These studies have also demonstrated that (1) the natural remanent magnetism of borderland sediments is primarily carried by detrital magnetite, although local contributions are sometimes made from other sources (e.g., detrital and authigenic hematite, altered glauconite, and biogenic magnetite); (2) the proportion of magnetic material decreases outward from the coastline because of dilution from biogenic sources; (3) the average magnetic grain size decreases away from the coastline; and (4) magnetic dissolution caused by early sediment diagenesis is an ubiquitous process, especially in the deeper basins (see summary provided by Lund et al., 1992). These observations were primarily derived from studies of Holocene-age cores. It remains to be seen if they are equally valid for interpreting older sedimentary records from these basins.

\section{METHODS}

\section{Introduction}

Magnetic susceptibility measurements were made on whole-core (shipboard) and split-core (shore-based) sections recovered from Site 893. Additionally, discrete measurements of index properties, calcium carbonate content, and magnetic susceptibility were made on samples taken at $\sim 75-\mathrm{cm}$ spacing. These measurements are used to define the general trends and relationships in downhole physical properties (bulk density, porosity, and water content) and magnetic properties in samples from Hole 893A.

The discrete index properties and magnetic susceptibility measurements (at $\sim 75 \mathrm{~cm}$ sample spacing) are used, along with measurements of magnetic susceptibility at $5-\mathrm{cm}$ spacing, to provide a range of sampling resolution which is intended to adequately portray the first order intermittently laminated nature of the sediment in Hole $893 \mathrm{~A}$. We will concentrate on the characteristics of the magnetic susceptibility record in Hole 893A. In later discussions, the magnetic susceptibility data are used to correlate Hole $893 \mathrm{~A}$ with the complementary sequence recovered in Hole $893 \mathrm{~B}$ and to transfer the Hole $893 \mathrm{~A}$ age model to Hole 893B. A more detailed discussion of these types of interhole correlations, using magnetic susceptibility and digital color data from the upper $24 \mathrm{~m}$ of each hole, is provided by Rack and Merrill (this volume).

\section{Magnetic Susceptibility Measurements}

\section{Near-Continuous Measurements}

Sensors mounted on the multisensor track (MST) aboard the JOIDES Resolution were used to collect closely spaced measurements of bulk density and magnetic susceptibility (using an $80 \mathrm{~mm}$ Bartington susceptibility loop) over five intervals in Hole 893A. The five measured intervals include: Core 146-893A-1H through Section $146-893 \mathrm{~A}-3 \mathrm{H}-1$ (0-17.5 mbsf) and Cores 146-893A-5H (35.0-44.5 


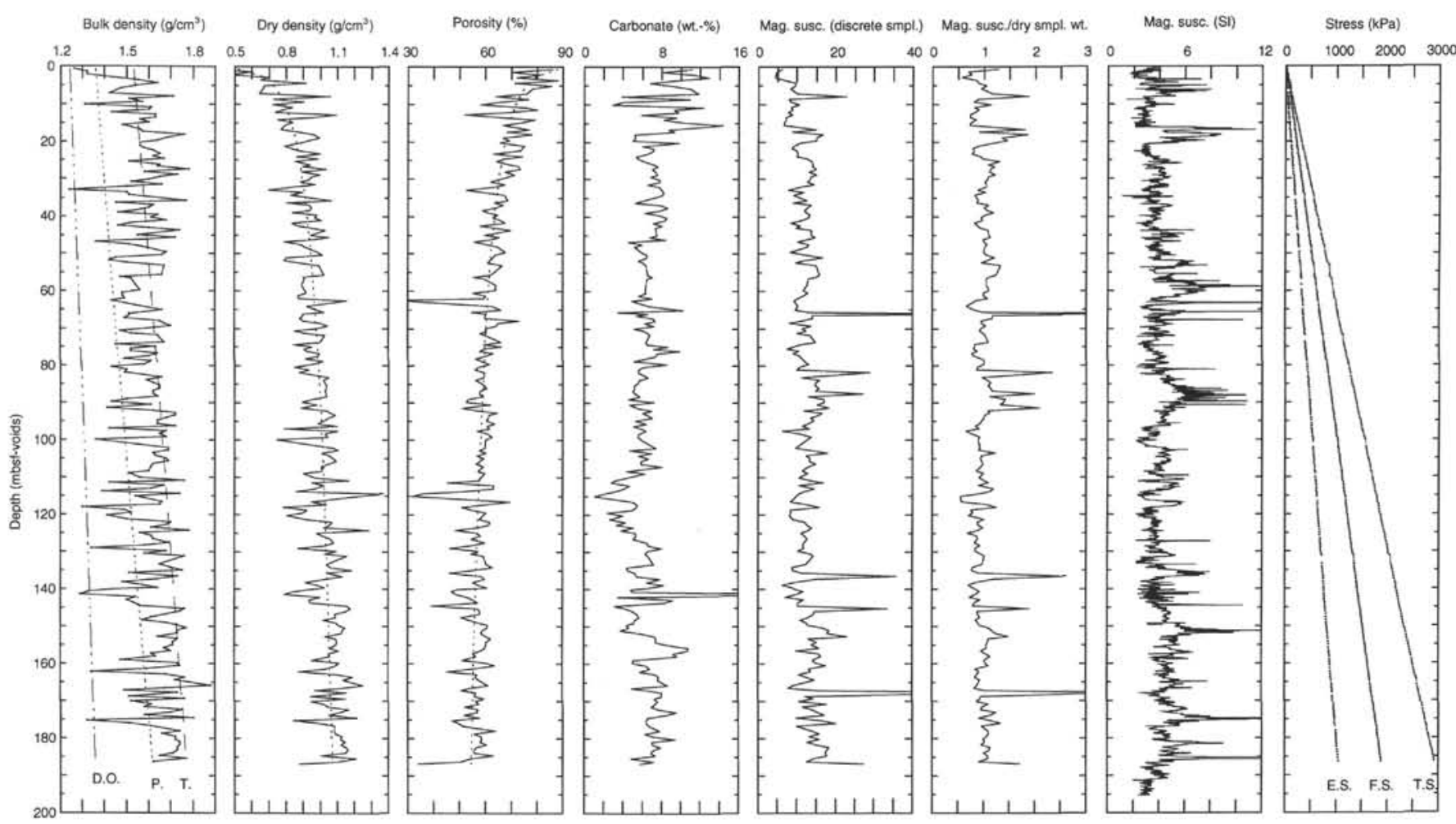

Figure 2. Summary plots of measurements of index properties, carbonate content, magnetic susceptibility, and vertical effective stress calculations from Hole 893A. Data are plotted vs. void-corrected depth. Empirical curves (after Hamilton, 1976) for diatom ooze (DO), pelagic clay (P), and terrigenous silts (T) are plotted with the bulk density measurements. Note: $\mathrm{ES}=$ vertical effective stress, $\mathrm{FS}=$ fluid stress, and TS $=$ total stress in kilopascals $(\mathrm{kPa})$.

mbsf), 10H (82.5-92.0 mbsf), 15H (130.0-139.5 mbsf), and 146$893 \mathrm{~A}-20 \mathrm{H}$ (168.0-177.5 mbsf), respectively.

Extensive voids were created in the Site 893 cores by gas expansion, as the cores were recovered on deck. The presence of these voids created difficulties in obtaining high quality data within some of the measured intervals, both for the MST sensors on the ship and for later shore-based measurements. The shore-based measurements of magnetic susceptibility were made (using a 100-mm Bartington susceptibility loop), following the shipment of the cores from the JOIDES Resolution to the ODP Gulf Coast Repository, in conjunction with visual core descriptions and other kinds of analyses (biostratigraphic studies, inorganic and organic geochemistry, etc.) by members of the Santa Barbara Basin Shore-based Scientific Party (Kennett, Baldauf, et al., 1994). Careful comparisons between shorebased core photographs and digital color images were subsequently used to identify the position of voids within individual core sections (see void tables in Kennett, Baldauf, et al., 1994; Merrill and Beck, this volume); these identifications were used to remove most of the void-related effects from both the shipboard and shore-based data.

Magnetic susceptibility measurements were routinely made on split-core sections from Holes $893 \mathrm{~A}$ and $893 \mathrm{~B}$ at approximately $5-\mathrm{cm}$ intervals using a Bartington 100-mm magnetic susceptibility loop set at the low frequency $(0.47 \mathrm{kHz})$ scale. Background measurements were taken at $10 \mathrm{~cm}$ from the ends (top and bottom) of each core section and data quality was monitored throughout the collection process. Measurements taken within $5 \mathrm{~cm}$ of any significant core disturbance or void were removed from the data set to avoid edge-effects or other sources of erroneous data. These data are given along with their respective age assignments in the appendixes of this paper, which are available on the CD-ROM accompanying this volume; Appendix A contains the data for Hole 893A, and Appendix B contains the data for Hole 893B.

\section{Discrete Measurements}

Discrete measurements of magnetic susceptibility were obtained from variable-volume sample plugs that were placed inside a $36-\mathrm{mm}$ (ID) loop attached to a Bartington MS-2 magnetic susceptibility meter. Duplicate measurements collected from each sample were shown to be very consistent. The methods used to collect these data are described in Kennett, Baldauf, et al. (1994). In this paper, the discrete susceptibility data are presented as raw values and as ratios of raw values to dry sample weight (determined by dividing the measured susceptibility of individual samples by their dry weight; see table 4 in Kennett, Baldauf, et al., 1994); these values are compared with the whole-core and split-core susceptibility data measured at a much closer along-core sample resolution (Fig. 2).

\section{Index Properties}

Index properties (wet and dry bulk density, grain density, porosity, and water content) were calculated for 242 discrete sediment samples from the Hole 893A cores (magnetic susceptibility measurements were previously measured on these same samples). Fifty-six of the discrete samples were selected from the main group in order to determine their respective index properties using a Penta pycnometer located at the ODP Gulf Coast Repository. These data are tabulated in Kennett, Baldauf, et al. (1994), where a more detailed description of the methodology used can be found.

The bulk density determined for each sample was used to calculate the cumulative increase in total vertical stress, fluid stress, and vertical effective stress, respectively, for each of the 242 sampled intervals in Hole 893A (Table 1; Fig. 2).

The vertical effective stress (in kilopascals, $\mathrm{kPa}$ ) at a given depth is equal to the sum of the incremental stresses for each layer above 
Table 1. Laboratory determinations of calcium carbonate, calculations of normalized magnetic susceptibility, and effective stress calculations, Hole 893A.

\begin{tabular}{|c|c|c|c|c|c|c|c|c|c|}
\hline $\begin{array}{l}\text { Core, section, } \\
\text { interval }(\mathrm{cm})\end{array}$ & $\begin{array}{l}\text { Raw depth } \\
\text { (mbsf) }\end{array}$ & $\begin{array}{l}\text { Void- } \\
\text { corrected } \\
\text { depth } \\
\text { (mbsf) }\end{array}$ & $\begin{array}{l}\text { Age } \\
\text { (k.y.) }\end{array}$ & $\begin{array}{l}\mathrm{CaCO}_{3} \\
(\mathrm{wt} \%)\end{array}$ & $\begin{array}{l}\text { MS } \\
\text { (SI) }\end{array}$ & MS* & $\begin{array}{c}\mathrm{TS} \\
(\mathrm{kPa})\end{array}$ & $\begin{array}{c}\mathrm{FS} \\
(\mathrm{kPa})\end{array}$ & $\begin{array}{c}\mathrm{ES} \\
(\mathrm{kPa})\end{array}$ \\
\hline \multicolumn{10}{|l|}{$146-893 \mathrm{~A}-$} \\
\hline $1 \mathrm{H}-1.57-59$ & 0.58 & 0.58 & 0.268 & 11.13 & 6.1 & 1.3 & 16 & 13 & 3 \\
\hline IH-I, 132-134 & 1.33 & 1.33 & 0.614 & 7.99 & 4.7 & 0.7 & 26 & 21 & 5 \\
\hline $\mathrm{IH}-2,58-60$ & 2.09 & 2.09 & 0.964 & 11.41 & 4.4 & 0.8 & 36 & 28 & 7 \\
\hline IH $-2,132-134$ & 2.83 & 2.83 & 1.306 & 12.89 & 4.2 & 0.6 & 47 & 36 & 10 \\
\hline $\mathrm{IH}-3,58-60$ & 3.61 & 3.59 & 1.656 & 9.39 & 6.7 & 0.8 & 58 & 44 & 14 \\
\hline IH-3, $132-134$ & 4.35 & 4.33 & 2.054 & 6.76 & 9.7 & 0.9 & 70 & 51 & 19 \\
\hline IH $4,58-60$ & 5.11 & 5.09 & 2.466 & 9.12 & 9.5 & 1.0 & 81 & 58 & 23 \\
\hline IH- $4,132-134$ & 5.85 & 5.81 & 2.937 & 10.94 & 8.2 & 0.9 & 99 & 71 & 28 \\
\hline $2 \mathrm{H}-1,57-59$ & 7.08 & 7.06 & 4.339 & 11.79 & 10.0 & 1.1 & 109 & 79 & 31 \\
\hline $2 \mathrm{H}-\mathrm{I}, 133-135$ & 7.84 & 7.82 & 5.192 & 3.82 & 22.7 & 1.9 & 122 & 86 & 36 \\
\hline $2 \mathrm{H}-2,57-59$ & 8.62 & 8.58 & 6.044 & 10.90 & 7.8 & 0.9 & 133 & 93 & 39 \\
\hline $2 \mathrm{H}-2,133-135$ & 9.38 & 9.30 & 6.543 & 3.98 & 8.3 & 0.8 & 144 & 100 & 43 \\
\hline $2 \mathrm{H}-3,57-59$ & 10.12 & 9.99 & 6.957 & 2.90 & 10.5 & 1.1 & 153 & 108 & 45 \\
\hline $2 \mathrm{H}-3,133-135$ & 10.88 & 10.72 & 7.394 & 12.31 & 8.4 & 0.8 & 163 & 114 & 49 \\
\hline $2 \mathrm{H}-4,57-59$ & 11.63 & 11.37 & 7.783 & 9.25 & 8.8 & 0.9 & 174 & 121 & 53 \\
\hline $2 \mathrm{H}-4,133-135$ & 12.39 & 12.06 & 8.196 & 9.69 & 7.7 & 0.8 & 183 & 128 & 55 \\
\hline $2 \mathrm{H}-5,62-64$ & 13.18 & 12.71 & 8.585 & 5.86 & 8.8 & 0.7 & 194 & 134 & 59 \\
\hline $2 \mathrm{H}-5,133-135$ & 13.89 & 13.36 & 8.975 & 9.48 & 7.1 & 0.7 & 204 & 141 & 63 \\
\hline $2 \mathrm{H}-6,57-59$ & 14.63 & 14.01 & 9.358 & 8.18 & 7.1 & 0.8 & 215 & 148 & 67 \\
\hline $2 \mathrm{H}-6,133-135$ & 15.39 & 14.76 & 9.688 & 10.36 & 6.7 & 0.7 & 227 & 156 & 71 \\
\hline $2 \mathrm{H}-7.57-59$ & 16.13 & 15.50 & 10.014 & 14.36 & 6.6 & 0.7 & 243 & 167 & 76 \\
\hline $3 \mathrm{H}-\mathrm{I}, 64-66$ & 16.65 & 16.62 & 10.507 & 8.70 & 14.9 & 1.8 & 254 & 174 & 80 \\
\hline $3 \mathrm{H}-1,132-134$ & 17.33 & 17.30 & 10.947 & 9.32 & 8.8 & 0.9 & 265 & 182 & 84 \\
\hline $3 \mathrm{H}-2,58-60$ & 18.09 & 18.06 & 11.439 & 5.17 & 16.6 & 1.8 & 278 & 189 & 89 \\
\hline $3 \mathrm{H}-2,132-134$ & 18.83 & 18.80 & 11.855 & 5.29 & 14.9 & 1.3 & 292 & 197 & 94 \\
\hline $3 \mathrm{H}-3,64-66$ & 19.65 & 19.62 & 12.431 & 5.02 & 15.0 & 1.4 & 301 & 203 & 98 \\
\hline $3 \mathrm{H}-3,126-128$ & 20.27 & 20.20 & 12838 & 9.81 & 10.9 & 1.2 & 314 & 212 & 102 \\
\hline $3 \mathrm{H}-4,65-67$ & 21.16 & 21.07 & 13.175 & 6.02 & 9.1 & 0.9 & 324 & 219 & 106 \\
\hline $3 \mathrm{H}-4,132-134$ & 21.83 & 21.74 & 13.376 & 7.07 & 8.7 & 0.8 & 334 & 225 & 109 \\
\hline $3 \mathrm{H}-5,51-53$ & 22.52 & 22.35 & 13.619 & 7.16 & 9.8 & 0.8 & 347 & 233 & 114 \\
\hline $3 \mathrm{H}-5,143-145$ & 23.44 & 23.15 & 13.977 & 6.47 & 10.2 & 0.8 & 357 & 239 & 118 \\
\hline $3 \mathrm{H}-6,63-65$ & 24.14 & 23.80 & 14.268 & 5.34 & 10.0 & 0.9 & 368 & 246 & 122 \\
\hline $3 \mathrm{H}-6,132-134$ & 24.83 & 24.49 & 14.881 & 5.56 & 12.7 & 1.0 & 380 & 253 & 127 \\
\hline $3 \mathrm{H}-7,55-57$ & 25.56 & 25.21 & 15.797 & 6.26 & 13.8 & 1.3 & 393 & 262 & 131 \\
\hline $4 \mathrm{H}-1,60-62$ & 26.11 & 26.11 & 16.528 & 7.28 & 14.0 & 1.1 & 401 & 267 & 134 \\
\hline $4 \mathrm{H}-\mathrm{I}, 132-134$ & 26.83 & 26.59 & 16.765 & 7.53 & 14.0 & 1.2 & 412 & 274 & 138 \\
\hline $4 \mathrm{H}-2,58-60$ & 27.59 & 27.29 & 17.110 & 7.00 & 14.9 & 1.1 & 425 & 281 & 143 \\
\hline $4 \mathrm{H}-2,134-136$ & 28.34 & 28.00 & 17.460 & 7.36 & 13.1 & 1.1 & 436 & 289 & 147 \\
\hline $4 \mathrm{H}-3,57-59$ & 29.08 & 28.74 & 17.825 & 7.52 & 15.1 & 1.2 & 447 & 296 & 152 \\
\hline $4 \mathrm{H}-3,131-133$ & 29.82 & 29.40 & 18.150 & 6.88 & 13.5 & 1.1 & 458 & 302 & 156 \\
\hline $4 \mathrm{H}-4,47-49$ & 30.48 & 30.03 & 18.812 & 7.79 & 13.3 & 1.1 & 467 & 308 & 159 \\
\hline $4 \mathrm{H}-4,124-126$ & 31.25 & 30.66 & 19.649 & 7.78 & 12.6 & 1.0 & 478 & 316 & 163 \\
\hline $4 \mathrm{H}-5,57-59$ & 32.08 & 31.40 & 20.632 & 6.54 & 14.4 & 1.0 & 488 & 322 & 167 \\
\hline $4 \mathrm{H}-5,126-128$ & 32.77 & 32.00 & 21.126 & 7.71 & 11.0 & 1.0 & 501 & 330 & 170 \\
\hline $4 \mathrm{H}-6,62-64$ & 33.63 & 32.84 & 21.710 & 7.98 & 7.8 & 0.9 & 509 & 337 & 172 \\
\hline $4 \mathrm{H}-6,134-136$ & 34.35 & 33.51 & 22.176 & 8.20 & 11.8 & 1.0 & 519 & 344 & 175 \\
\hline $4 \mathrm{H}-7,57-59$ & 35.08 & 34.19 & 22.649 & 8.08 & 9.1 & 0.8 & 539 & 358 & 182 \\
\hline $5 \mathrm{H}-1.57-59$ & 35.58 & 35.58 & 23.616 & 6.03 & 12.5 & 0.9 & 550 & 364 & 186 \\
\hline $5 \mathrm{H}-\mathrm{I}, 132-134$ & 36.33 & 36.19 & 24.041 & 5.24 & 8.9 & 0.9 & 559 & 370 & 189 \\
\hline $5 \mathrm{H}-2,57-59$ & 37.08 & 36.80 & 24.465 & 7.92 & 13.6 & 1.1 & 571 & 377 & 193 \\
\hline $5 \mathrm{H}-2,132-134$ & 37.83 & 37.55 & 24.987 & 8.55 & 12.6 & 1.0 & 582 & 384 & 197 \\
\hline $5 \mathrm{H}-3,57-59$ & 38.58 & 38.25 & 25.473 & 7.80 & 11.9 & 1.1 & 592 & 391 & 200 \\
\hline $5 \mathrm{H}-3,132-134$ & 39.33 & 38.92 & 25.940 & 6.22 & 12.3 & 1.2 & 601 & 398 & 203 \\
\hline $5 \mathrm{H}-4,57-59$ & 40.08 & 39.56 & 26.385 & 7.62 & 13.3 & 1.0 & 612 & 405 & 207 \\
\hline $5 \mathrm{H}-4,132-134$ & 40.83 & 40.26 & 26.872 & 8.52 & 12.9 & 0.9 & 623 & 412 & 211 \\
\hline $5 \mathrm{H}-5,57-59$ & 41.58 & 40.97 & 27.366 & 8.29 & 11.2 & 1.0 & 636 & 419 & 216 \\
\hline $5 \mathrm{H}-5,132-134$ & 42.33 & 41.72 & 27.887 & 7.08 & 8.4 & 0.8 & 645 & 426 & 219 \\
\hline 5H-6, 57-59 & 43.08 & 42.36 & 28.333 & 8.01 & 10.9 & 0.9 & 654 & 432 & 222 \\
\hline $5 \mathrm{H}-6,132-134$ & 43.83 & 42.96 & 28.750 & 7.34 & 9.6 & 0.9 & 665 & 439 & 226 \\
\hline $5 \mathrm{H}-7,57-59$ & 44.58 & 43.65 & 29.241 & 7.39 & 13.7 & 1.1 & 687 & 452 & 235 \\
\hline $6 \mathrm{H}-1.57-59$ & 45.08 & 44.93 & 30.162 & 7.47 & 13.6 & 1.1 & 695 & 457 & 238 \\
\hline $6 \mathrm{H}-1.132-134$ & 45.83 & 45.50 & 30.573 & 6.73 & 14.6 & 1.1 & 705 & 463 & 242 \\
\hline $6 \mathrm{H}-2,57-59$ & 46.58 & 46.06 & 30.975 & 8.38 & 12.5 & 1.0 & 715 & 470 & 245 \\
\hline $6 \mathrm{H}-2,132-134$ & 47.33 & 46.75 & 31.472 & 4.55 & 10.1 & 1.0 & 725 & 477 & 248 \\
\hline $6 \mathrm{H}-3,57-59$ & 48.08 & 47.48 & 31.997 & 5.95 & 12.9 & 1.1 & 734 & 483 & 251 \\
\hline $6 \mathrm{H}-3,132-134$ & 48.83 & 48.08 & 32.429 & 5.16 & 12.6 & 1.0 & 756 & 498 & 258 \\
\hline $6 \mathrm{H}-4,132-134$ & 50.33 & 49.52 & 33.465 & 5.92 & 12.4 & 1.0 & 768 & 505 & 263 \\
\hline $6 \mathrm{H}-5,57-59$ & 51.08 & 50.23 & 33.976 & 6.45 & 16.7 & 1.2 & 780 & 512 & 268 \\
\hline $6 \mathrm{H}-5,132-134$ & 51.83 & 50.98 & 34.515 & 6.43 & 11.7 & 1.1 & 790 & 519 & 270 \\
\hline $6 \mathrm{H}-6,58-60$ & 52.59 & 51.67 & 35.012 & 5.58 & 9.6 & 0.9 & 799 & 526 & 273 \\
\hline $6 \mathrm{H}-6,132-134$ & 53.33 & 52.32 & 35.480 & 6.06 & 14.9 & 1.3 & 810 & 533 & 276 \\
\hline $6 \mathrm{H}-7,57-59$ & 54.08 & 53.07 & 36.019 & 6.35 & 15.8 & 1.2 & 851 & 559 & 292 \\
\hline $7 \mathrm{H}-3,57-59$ & 56.16 & 55.60 & 37.840 & 6.55 & 14.9 & 1.1 & 860 & 564 & 296 \\
\hline $7 \mathrm{H}-3,132-134$ & 56.91 & 56.14 & 38.228 & 7.05 & 12.9 & 1.0 & 867 & 569 & 298 \\
\hline $7 \mathrm{H}-4,57-59$ & 57.66 & 56.61 & 38.567 & 6.47 & 12.6 & 1.0 & 890 & 584 & 305 \\
\hline $7 \mathrm{H}-5,57-59$ & 59.23 & 58.14 & 39.667 & 6.39 & 11.6 & 1.1 & 911 & 599 & 313 \\
\hline $7 \mathrm{H}-6,57-59$ & 60.73 & 59.58 & 40.703 & 6.32 & 12.8 & 1.0 & 923 & 606 & 316 \\
\hline $7 \mathrm{H}-6,132-134$ & 61.48 & 60.33 & 41.243 & 6.34 & 11.7 & 1.0 & 934 & 614 & 320 \\
\hline $7 \mathrm{H}-7,57-59$ & 62.23 & 61.07 & 41.776 & 5.65 & 10.3 & 1.1 & 945 & 621 & 323 \\
\hline $7 \mathrm{H}-7,132-134$ & 62.98 & 61.82 & 42.315 & 6.97 & 9.2 & 0.8 & 954 & 628 & 326 \\
\hline $7 \mathrm{H}-8,57-59$ & 63.73 & 62.49 & 42.797 & 4.87 & 10.3 & 0.7 & 974 & 642 & 332 \\
\hline $8 \mathrm{H}-2,24-26$ & 63.93 & 63.90 & 43.812 & 6.76 & 9.6 & 0.8 & 991 & 653 & 338 \\
\hline $8 \mathrm{H}-2,132-134$ & 65.01 & 64.98 & 44.589 & 10.20 & 12.9 & 1.0 & 999 & 658 & 341 \\
\hline $8 \mathrm{H}-3,58-60$ & 65.77 & 65.49 & 44.956 & 3.51 & 45.1 & 3.2 & 1007 & 664 & 344 \\
\hline
\end{tabular}


Table 1 (continued).

\begin{tabular}{|c|c|c|c|c|c|c|c|c|}
\hline $\begin{array}{l}\text { Core, section. } \\
\text { interval }(\mathrm{cm})\end{array}$ & $\begin{array}{l}\text { Raw depth } \\
\text { (mbsf) }\end{array}$ & $\begin{array}{l}\text { Void- } \\
\text { corrected } \\
\text { depth } \\
\text { (mbsf) }\end{array}$ & $\begin{array}{l}\text { Age } \\
\text { (k.y.) }\end{array}$ & $\begin{array}{l}\mathrm{CaCO}_{3} \\
\text { (wt } \% \text { ) }\end{array}$ & $\begin{array}{l}\text { MS } \\
\text { (SI) }\end{array}$ & MS* & $\begin{array}{c}\text { TS } \\
(\mathrm{kPa})\end{array}$ & $\begin{array}{c}\text { FS } \\
(\mathrm{kPa})\end{array}$ \\
\hline $8 \mathrm{H}-3,134-136$ & 66.53 & 66.02 & 45.337 & 6.51 & 13.9 & 1.2 & 1013 & 667 \\
\hline $8 \mathrm{H}-4,57-59$ & 67.26 & 66.38 & 45.596 & 5.37 & 14.0 & 1.2 & 1023 & 674 \\
\hline $8 \mathrm{H}-4,132-134$ & 68.01 & 67.08 & 46.100 & 7.11 & 11.1 & 0.9 & 1034 & 682 \\
\hline $8 \mathrm{H}-5,60-62$ & 68.79 & 67.83 & 46.640 & 7.30 & 8.1 & 1.0 & 1045 & 689 \\
\hline $8 \mathrm{H}-5,132-134$ & 69.51 & 68.52 & 47.136 & 6.27 & 13.7 & 1.0 & 1057 & 696 \\
\hline $8 \mathrm{H}-6,53-55$ & 70.22 & 69.22 & 47.640 & 7.21 & 11.3 & 0.9 & 1068 & 703 \\
\hline $8 \mathrm{H}-6,132-134$ & 71.01 & 69.92 & 48.144 & 5.17 & 12.3 & 0.9 & 1078 & 709 \\
\hline $8 \mathrm{H}-7,59-61$ & 71.78 & 70.57 & 48.611 & 6.20 & 10.1 & 1.0 & 1088 & 716 \\
\hline $8 \mathrm{H}-7,132-134$ & 72.51 & 71.24 & 49.093 & 6.63 & 13.4 & 1.0 & 1096 & 721 \\
\hline $8 \mathrm{H}-8,57-59$ & 73.26 & 71.76 & 49.467 & 6.76 & 14.7 & 1.1 & 1125 & 740 \\
\hline $9 \mathrm{H}-1.56-58$ & 73.57 & 73.57 & 50.770 & 6.33 & 10.9 & 0.8 & 1134 & 745 \\
\hline $9 \mathrm{H}-1,13 \mathrm{I}-133$ & 74.32 & 74.13 & 51.202 & 6.56 & 9.3 & 0.8 & 1143 & 752 \\
\hline $9 \mathrm{H}-2,56-58$ & 75.07 & 74.79 & 51.759 & 8.60 & 7.5 & 0.8 & 1153 & 758 \\
\hline $9 \mathrm{H}-2,131-133$ & 75.82 & 75.40 & 52.273 & 7.21 & 10.6 & 0.9 & 1162 & 764 \\
\hline $9 \mathrm{H}-3,56-58$ & 76.57 & 75.99 & 52.771 & 9.90 & 9.1 & 0.8 & 1170 & 769 \\
\hline $9 \mathrm{H}-3,124-126$ & 77.25 & 76.55 & 53.243 & 7.53 & 10.7 & 0.8 & 1182 & 777 \\
\hline $9 \mathrm{H}-4,56-58$ & 78.07 & 77.29 & 53.868 & 7.41 & 11.4 & 1.0 & 1193 & 784 \\
\hline $9 \mathrm{H}-4,13 \mathrm{I}-133$ & 78.82 & 77.97 & 54.441 & 6.19 & 11.8 & 1.0 & 1204 & 791 \\
\hline $9 \mathrm{H}-5,56-58$ & 79.57 & 78.70 & 55.057 & 5.10 & 12.9 & 1.0 & 1215 & 798 \\
\hline $9 \mathrm{H}-5,13 \mathrm{I}-133$ & 80.32 & 79.43 & 55.672 & 8.50 & 10.7 & 0.9 & 1228 & 807 \\
\hline $9 \mathrm{H}-7.56-58$ & 81.28 & 80.25 & 56.364 & 6,01 & 9.9 & 0.8 & 1238 & 814 \\
\hline $9 \mathrm{H}-7,13 \mathrm{I}-133$ & 82.03 & 80.94 & 56.946 & 5.54 & 28.8 & 2.3 & 1249 & 821 \\
\hline $9 \mathrm{H}-8,56-58$ & 82.78 & 81.69 & 57.578 & 5.56 & 11.1 & 1.0 & 1268 & 835 \\
\hline $10 \mathrm{H}-1,57-59$ & 83.08 & 83.05 & 58.725 & 6.58 & 15.7 & 1.1 & 1279 & 842 \\
\hline $10 \mathrm{H}-1,128-130$ & 83.79 & 83.72 & 59.290 & 5.62 & 14.7 & 1.1 & 1291 & 849 \\
\hline $10 \mathrm{H}-2,57-59$ & 84.58 & 84.44 & 59.898 & 5.56 & 14.6 & 1.1 & 1302 & 856 \\
\hline $10 \mathrm{H}-2,132-134$ & 85.33 & 85.19 & 60.530 & 5.12 & 15.6 & 1.1 & 1314 & 864 \\
\hline $\mathrm{IOH}-3,57-59$ & 86.08 & 85.93 & 61.154 & 5.18 & 14.8 & 1.2 & 1327 & 871 \\
\hline $10 \mathrm{H}-3,132-134$ & 86.83 & 86.68 & 61.787 & 5.01 & 27.0 & 2.0 & 1340 & 880 \\
\hline $10 \mathrm{H}-4,59-61$ & 87.70 & 87.52 & 62.495 & 5.31 & 12.9 & 1.1 & 1351 & 887 \\
\hline $10 \mathrm{H}-4,132-134$ & 88.43 & 88.24 & 63.102 & 5.72 & 17.0 & 1.4 & 1363 & 894 \\
\hline $10 \mathrm{H}-5,57-59$ & 89.18 & 88.98 & 63.727 & 4.57 & 17.0 & 1.4 & 1374 & 902 \\
\hline $10 \mathrm{H}-5,132-134$ & 89.93 & 89.73 & 64.359 & 7.18 & 15.1 & 1.3 & 1384 & 909 \\
\hline $10 \mathrm{H}-6,54-56$ & 90.65 & 90.40 & 64.924 & 4.66 & 18.2 & 2.1 & 1397 & 917 \\
\hline $10 \mathrm{H}-7,57-59$ & 91.51 & 91.23 & 65.791 & 5.52 & 11.8 & 1.1 & 1407 & 925 \\
\hline $10 \mathrm{H}-7,132-134$ & 92.26 & 91.98 & 66.592 & 6.95 & 16.2 & 1.1 & 1416 & 930 \\
\hline $11 \mathrm{H}-1,57-59$ & 92.58 & 92.56 & 67.212 & 6.03 & 15.6 & 1.0 & 1428 & 937 \\
\hline $1 \mathrm{IH}-\mathrm{I}, 132-134$ & 93.33 & 93.22 & 67.917 & 6.55 & 13.5 & 1.0 & 1440 & 945 \\
\hline $11 \mathrm{H}-2,57-59$ & 94.08 & 93.97 & 68.718 & 7.04 & 14.3 & 1.0 & 1452 & 952 \\
\hline $11 \mathrm{H}-2,127-129$ & 94.78 & 94.67 & 69.466 & 5.28 & 12.8 & 0.9 & 1465 & 960 \\
\hline $11 \mathrm{H}-3,57-59$ & 95.58 & 95.47 & 70.321 & 6.42 & 11.3 & 0.8 & 1476 & 967 \\
\hline $11 \mathrm{H}-3,127-129$ & 96.28 & 96.17 & 71.069 & 5.04 & 13.2 & 0.9 & 1488 & 974 \\
\hline $11 \mathrm{H}-4,57-59$ & 97.08 & 96.88 & 71.828 & 5.95 & 6.2 & 0.7 & 1496 & 979 \\
\hline $11 \mathrm{H}-4,127-129$ & 97.78 & 97.44 & 72.426 & 6.38 & 10.7 & 0.8 & 1509 & 987 \\
\hline $11 \mathrm{H}-5,57-59$ & 98.58 & 98.23 & 73.270 & 5.59 & 13.7 & 0.9 & 1521 & 995 \\
\hline $11 \mathrm{H}-6,57-59$ & 99.35 & 98.99 & 74.082 & 5.50 & 12.7 & 0.9 & 1533 & 1003 \\
\hline $11 \mathrm{H}-6,132-134$ & 100.10 & 99.74 & 74.884 & 5.92 & 9.6 & 0.9 & 1563 & 1025 \\
\hline $12 \mathrm{H}-1,57-59$ & 102.08 & 101.97 & 77.266 & 7.37 & 13.3 & 0.9 & 1575 & 1032 \\
\hline $12 \mathrm{H}-\mathrm{I}, 132-134$ & 102.83 & 102.66 & 78.004 & 4.63 & 17.9 & 1.2 & 1587 & 1039 \\
\hline $12 \mathrm{H}-2,57-59$ & 103.58 & 103.38 & 78.773 & 6.91 & 12.4 & 1.0 & 1597 & 1046 \\
\hline $12 \mathrm{H}-2,132-134$ & 104.33 & 104.07 & 79.510 & 5.60 & 13.4 & 0.9 & 1610 & 1054 \\
\hline $12 \mathrm{H}-3,57-59$ & 105.15 & 104.84 & 80.333 & 6.65 & 13.2 & 1.0 & 1622 & 1062 \\
\hline $12 \mathrm{H}-3,133-135$ & 105,91 & 105,60 & 81.145 & 5.87 & 14.7 & 1.0 & 1635 & 1069 \\
\hline $12 \mathrm{H}-4,64-66$ & 106.74 & 106.35 & 81.947 & 5.84 & 12.9 & 0.9 & 1645 & 1076 \\
\hline $12 \mathrm{H}-4,132-134$ & 107.42 & 107.00 & 82.641 & 8.04 & 11.3 & 0.8 & 1664 & 1088 \\
\hline $12 \mathrm{H}-6,57-59$ & 108.63 & 108.21 & 83.934 & 4.39 & 12.8 & 1.0 & 1673 & 1094 \\
\hline $12 \mathrm{H}-6,132-134$ & 109.38 & 108.79 & 84.554 & 6.26 & 10.5 & 0.9 & 1690 & 1105 \\
\hline $12 \mathrm{H}-8,58-60$ & 110.55 & 109.93 & 85.772 & 4.34 & 13.3 & 1.0 & 1701 & 1112 \\
\hline $12 \mathrm{H}-8,128-130$ & 111.25 & 110.63 & 86.520 & 3.13 & 16.7 & 1.1 & 1713 & 1119 \\
\hline $\mid 3 \mathrm{H}-1,59-61$ & 111.60 & 111.30 & 87.236 & 2.79 & 10.5 & 0.9 & 1722 & 1126 \\
\hline $13 \mathrm{H}-1,13 \mathrm{l}-133$ & 112.32 & 111.97 & 87.951 & 4.66 & 14.5 & 1.2 & 1738 & 1135 \\
\hline $13 \mathrm{H}-3,56-58$ & 113.29 & 112.93 & 88.977 & 4.05 & 12.0 & 1.1 & 1749 & 1143 \\
\hline $13 \mathrm{H}-3,13 \mathrm{l}-133$ & 114.04 & 113.67 & 89.768 & 3.12 & 10.5 & 0.9 & 1756 & 1148 \\
\hline $13 \mathrm{H}-4,56-58$ & 114.72 & 114.16 & 90.003 & 2.03 & 9.8 & 0.5 & 1769 & 1155 \\
\hline $13 \mathrm{H}-4,13 \mathrm{I}-133$ & 115.47 & 114.91 & 90.003 & 1.07 & 8.4 & 0.6 & 1791 & 1170 \\
\hline $13 \mathrm{H}-5,13 \mathrm{I}-133$ & 116.97 & 116.40 & 90.046 & 5.02 & 12.6 & 1.1 & 1804 & 1178 \\
\hline $13 \mathrm{H}-6,56-58$ & 117.72 & 117.16 & 90.858 & 5.45 & 15.8 & 1.2 & 1816 & 1185 \\
\hline $13 \mathrm{H}-6,13 \mathrm{I}-133$ & 118.47 & 117.91 & 91.659 & 5.33 & 8.4 & 0.8 & 1825 & 1193 \\
\hline $13 \mathrm{H}-7,56-58$ & 119.22 & 118.67 & 92.471 & 4.92 & 8.4 & 0.8 & 1836 & 1200 \\
\hline $13 \mathrm{H}-7,129-131$ & 119.95 & 119.40 & 93.251 & 2.38 & 8.1 & 0.7 & 1846 & 1207 \\
\hline $13 \mathrm{H}-8,56-58$ & 120.72 & 120.07 & 93.967 & 3.98 & 9.0 & 1.0 & 1860 & 1217 \\
\hline $14 \mathrm{H}-1,57-59$ & 121.08 & 121.08 & 95.046 & 2.62 & 12.1 & 0.9 & 1871 & 1224 \\
\hline $14 \mathrm{H}-1,133-135$ & 121.84 & 121.80 & 95.816 & 4.24 & 12.5 & 0.9 & 1884 & 1232 \\
\hline $14 \mathrm{H}-2,57-59$ & 122.65 & 122.60 & 96.671 & 3.36 & 13.7 & 1.0 & 1896 & 1240 \\
\hline $14 \mathrm{H}-2,132-134$ & 123.40 & 123.35 & 97.472 & 5.08 & 12.9 & 0.9 & 1909 & 1248 \\
\hline $14 \mathrm{H}-3,57-59$ & 124.22 & 124.14 & 98.316 & 3.78 & 11.1 & 0.7 & 1922 & 1256 \\
\hline $14 \mathrm{H}-3,133-135$ & 124.98 & 124.90 & 99.128 & 5.28 & 11.9 & 0.9 & 1933 & 1263 \\
\hline $14 \mathrm{H}-4,57-59$ & 125.72 & 125.63 & 99.908 & 5.35 & 12.6 & 0.9 & 1945 & 1270 \\
\hline $14 \mathrm{H}-4,133-135$ & 126.48 & 126.39 & 100.720 & 5.08 & 11.9 & 0.8 & 1963 & 1282 \\
\hline $14 \mathrm{H}-7,57-59$ & 127.65 & 127.51 & 101.917 & 6.51 & 11.5 & 0.9 & 1975 & 1289 \\
\hline $14 \mathrm{H}-7,132-134$ & 128.40 & 128.26 & 102.718 & 6.60 & 11.7 & 0.8 & 1986 & 1296 \\
\hline $14 \mathrm{H}-8,57-59$ & 129.15 & 128.95 & 103.456 & 8.01 & 10.2 & 0.8 & 1996 & 1304 \\
\hline $14 \mathrm{H}-8,132-134$ & 129.90 & 129.69 & 104.246 & 6.52 & 13.6 & 0.9 & 2007 & 1311 \\
\hline $15 \mathrm{H}-1,65-67$ & 130.66 & 130.38 & 104.984 & 6.32 & 14.2 & 1.0 & 2017 & 1317 \\
\hline $15 \mathrm{H}-1,131-133$ & 131.32 & 131.03 & 105.678 & 6.61 & 12.4 & 1.0 & 2056 & 1340 \\
\hline $15 \mathrm{H}-3,55-57$ & 133.59 & 133.29 & 108.093 & 7.16 & 8.6 & 0.8 & 2069 & 1347 \\
\hline $15 \mathrm{H}-3,131-133$ & 134.35 & 134.05 & 108.905 & 4.39 & 9.0 & 0.8 & 2081 & 1355 \\
\hline $15 \mathrm{H}-4,55-57$ & 135.13 & 134.77 & 109.674 & 4.46 & 11.1 & 0.9 & 2094 & 1362 \\
\hline $15 \mathrm{H}-4,131-133$ & 135.89 & 135.53 & 110.487 & 5.26 & 35.8 & 2.6 & 2107 & 1372 \\
\hline
\end{tabular}


Table 1 (continued).

\begin{tabular}{|c|c|c|c|c|c|c|c|c|c|}
\hline $\begin{array}{l}\text { Core, section, } \\
\text { interval }(\mathrm{cm})\end{array}$ & $\begin{array}{l}\text { Raw depth } \\
\text { (mbsf) }\end{array}$ & $\begin{array}{l}\text { Void- } \\
\text { corrected } \\
\text { depth } \\
\text { (mbsf) }\end{array}$ & $\begin{array}{l}\text { Age } \\
(k, y .)\end{array}$ & $\begin{array}{l}\mathrm{CaCO}_{3} \\
(\mathrm{wt} \%)\end{array}$ & $\begin{array}{l}\text { MS } \\
\text { (SI) }\end{array}$ & MS* & $\begin{array}{c}\text { TS } \\
(\mathrm{kPa})\end{array}$ & $\begin{array}{c}\mathrm{FS} \\
(\mathrm{kPa})\end{array}$ & $\begin{array}{c}\text { ES } \\
(\mathrm{kPa})\end{array}$ \\
\hline $15 \mathrm{H}-5.56-58$ & 136.75 & 136.44 & 111.459 & 5.37 & 15.2 & 1.2 & 2120 & 1379 & 741 \\
\hline $15 \mathrm{H}-5,13 \mathrm{I}-133$ & 137.50 & 137.19 & 112.260 & 7.83 & 9.4 & 0.9 & 2134 & 1388 & 746 \\
\hline $15 \mathrm{H}-6,56-58$ & 138.37 & 138.05 & 113.179 & 6.49 & 6.2 & 0.7 & 2145 & 1395 & 749 \\
\hline $15 \mathrm{H}-6,131-133$ & 139.12 & 138.80 & 113.980 & 8.23 & 9.0 & 0.8 & 2156 & 1403 & 753 \\
\hline $15 \mathrm{H}-7,56-58$ & 139.87 & 139.54 & 114.771 & 6.63 & 9.7 & 0.9 & 2163 & 1407 & 756 \\
\hline $16 \mathrm{H}-1.60-62$ & 140.11 & 139.96 & 115.220 & 4.79 & 11.7 & 0.9 & 2171 & 1413 & 758 \\
\hline $16 \mathrm{H}-1,131-133$ & 140.82 & 140.55 & 115.850 & 4.99 & 9.8 & 0.8 & 2180 & 1420 & 760 \\
\hline $16 \mathrm{H}-2,57-59$ & 141.58 & 141.26 & 116.552 & 19.69 & 7.1 & 0.7 & 2190 & 1427 & 762 \\
\hline $16 \mathrm{H}-2,131-133$ & 142.32 & 142.00 & 117.269 & 3.48 & 11.9 & 0.9 & 2201 & 1435 & 766 \\
\hline $16 \mathrm{H}-3,60-62$ & 143.11 & 142.77 & 118.016 & 9.17 & 10.1 & 0.8 & 2212 & 1442 & 770 \\
\hline $16 \mathrm{H}-3,13 \mathrm{I}-133$ & 143.82 & 143.48 & 118.703 & 8.30 & 10.2 & 0.8 & 2225 & 1451 & 774 \\
\hline $16 \mathrm{H}-4,55-57$ & 144.70 & 144.36 & 119.556 & 3.12 & 33.5 & 1.9 & 2236 & 1459 & 778 \\
\hline $16 \mathrm{H}-4,130-132$ & 145.45 & 145.11 & 120.283 & 4.40 & 14.5 & 0.9 & 2250 & 1467 & 784 \\
\hline $16 \mathrm{H}-5.57-59$ & 146.26 & 145.91 & 121.058 & 5.04 & 14.4 & 0.9 & 2263 & 1474 & 789 \\
\hline $16 \mathrm{H}-5,131-133$ & 147.00 & 146.65 & 121.775 & 5,48 & 12.9 & 0.9 & 2276 & 1482 & 794 \\
\hline $16 \mathrm{H}-6,57-59$ & 147.79 & 147.43 & 122.531 & 5.67 & 11.0 & 0.8 & 2288 & 1490 & 798 \\
\hline $16 \mathrm{H}-6,133-135$ & 148.55 & 148.19 & 122.904 & 5.16 & 12.5 & 0.9 & 2299 & 1497 & 802 \\
\hline $16 \mathrm{H}-7.57-59$ & 149.29 & 148.91 & 123.244 & 5.11 & 12.7 & 0.9 & 2310 & 1503 & 806 \\
\hline $17 \mathrm{H}-\mathrm{I}, 57-59$ & 149.58 & 149.56 & 123.551 & 4.35 & 16.7 & 1.1 & 2322 & 1511 & 811 \\
\hline $17 \mathrm{H}-1,131-133$ & 150.32 & 150.30 & 123.900 & 4.60 & 18.2 & 1.2 & 2335 & 1518 & 817 \\
\hline $17 \mathrm{H}-2,56-58$ & 151.07 & 151.03 & 124.244 & 3.69 & 17.7 & 1.2 & 2348 & 1526 & 822 \\
\hline $17 \mathrm{H}-2,131-133$ & 151.82 & 151.78 & 124.598 & 5.77 & 22.9 & 15 & 2361 & 1534 & 827 \\
\hline $17 \mathrm{H}-3,57-59$ & 152.61 & 152.57 & 124.971 & 7.33 & 12.9 & 1.1 & 2372 & 1541 & 832 \\
\hline $17 \mathrm{H}-3,131-133$ & 153.35 & 153.28 & 125.306 & 7.36 & 15.1 & i. & 2386 & 1549 & 837 \\
\hline $17 \mathrm{H}-4,56-58$ & 154.20 & 154.11 & 125.698 & 7.37 & 13.8 & 1.1 & 2399 & 1557 & 842 \\
\hline $17 \mathrm{H}-4,131-133$ & 154.95 & 154.86 & 126.052 & 8.55 & 15.7 & 1.0 & 2413 & 1565 & 848 \\
\hline $17 \mathrm{H}-5,56-58$ & 155.80 & 155.68 & 126.438 & 10.77 & 9.8 & 1.0 & 2425 & 1572 & 853 \\
\hline $17 \mathrm{H}-5,131-133$ & 156.55 & 156.43 & 126.792 & 10.68 & 15.6 & 1.0 & 2437 & 1580 & 857 \\
\hline $17 \mathrm{H}-6,56-58$ & 157.30 & 157.17 & 127.270 & 9.20 & 16.2 & 1.1 & 2449 & 1587 & 862 \\
\hline $17 \mathrm{H}-6,131-133$ & 158.05 & 157.88 & 127.910 & 9.61 & 14.1 & 1.0 & 2467 & 1599 & 868 \\
\hline $18 \mathrm{H}-\mathrm{I}, 53-55$ & 159.04 & 159.04 & 128.954 & 5.08 & 14.7 & 1.0 & 2478 & 1606 & 872 \\
\hline $18 \mathrm{H}-\mathrm{I}, 132-134$ & 159.83 & 159.78 & 129.621 & 4.97 & 17.5 & 1.1 & 2490 & 1613 & 877 \\
\hline $18 \mathrm{H}-2,57-59$ & 160.58 & 160.48 & 130.251 & 6.40 & 13.1 & 0.8 & 2503 & 1621 & 882 \\
\hline $18 \mathrm{H}-2,132-134$ & 161.33 & 161.23 & 130.927 & 6.78 & 13.7 & 0.9 & 2514 & 1628 & 886 \\
\hline $18 \mathrm{H}-3,57-59$ & 162.08 & 161.98 & 131.602 & 5.74 & 10.4 & 0.9 & 2524 & 1636 & 888 \\
\hline $18 \mathrm{H}-3,132-134$ & 162.83 & 162.73 & 132.277 & 7.42 & 12.5 & 0.8 & 2537 & 1644 & 893 \\
\hline $18 \mathrm{H}-4,57-59$ & 163.66 & 163.51 & 132.980 & 7.71 & 14.6 & 0.9 & 2550 & 1651 & 899 \\
\hline $18 \mathrm{H}-4,132-134$ & 164.41 & 164.26 & 133.656 & 7.61 & 9.2 & 0.9 & 2575 & 1666 & 909 \\
\hline $18 \mathrm{H}-5,132-134$ & 165.91 & 165.74 & 134.988 & 8.62 & 15.0 & 1.0 & 2588 & 1673 & 915 \\
\hline $18 \mathrm{H}-6,57-59$ & 166.66 & 166.45 & 135.628 & 4.86 & 55.6 & 3.5 & 2599 & 1680 & 919 \\
\hline $18 \mathrm{H}-6,132-134$ & 167.41 & 167.11 & 136.222 & 6.72 & 12.2 & 0.9 & 2606 & 1685 & 921 \\
\hline $18 \mathrm{H}-7.37-39$ & 167.96 & 167.64 & 136.699 & 7.98 & 14.3 & 0.9 & 2623 & 1695 & 928 \\
\hline $19 \mathrm{H}-\mathrm{I}, 62-64$ & 168.63 & 168.60 & 137.564 & 8.00 & 10.4 & 0.9 & 2633 & 1702 & 931 \\
\hline $19 \mathrm{H}-1.132-134$ & 169.33 & 169.30 & 138.195 & 7.29 & 17.6 & 1.1 & 2645 & 1709 & 936 \\
\hline $19 \mathrm{H}-2,57-59$ & 170.10 & 169.99 & 138.816 & 7.56 & 11.4 & 1.0 & 2655 & 1716 & 940 \\
\hline $19 \mathrm{H}-2,127-129$ & 170.80 & 170.69 & 139.446 & 7.25 & 13.9 & 0.9 & 2667 & 1723 & 944 \\
\hline $19 \mathrm{H}-3,57-59$ & 171.60 & 171.44 & 140.122 & 6.91 & 15.4 & 1.2 & 2679 & 1731 & 948 \\
\hline $19 \mathrm{H}-3,132-134$ & 172.35 & 172.17 & 140.779 & 7.75 & 17.0 & 1.1 & 2692 & 1738 & 953 \\
\hline $19 \mathrm{H}-4,57-59$ & 173.10 & 172.93 & 141.464 & 9.51 & 9.8 & 0.9 & 2704 & 1746 & 958 \\
\hline $19 \mathrm{H}-4,132-134$ & 173.85 & 173.68 & 142.139 & 8.40 & 16.4 & 1.1 & 2716 & 1754 & 962 \\
\hline $19 \mathrm{H}-5,57-59$ & 174.66 & 174.46 & 142.842 & 6.61 & 20.0 & 1.3 & 2729 & 1761 & 968 \\
\hline $19 \mathrm{H}-5,132-134$ & 175.41 & 175.21 & 143.517 & 6.36 & 10.0 & 1.0 & 2739 & 1769 & 970 \\
\hline $19 \mathrm{H}-6,57-59$ & 176.16 & 175.94 & 144.174 & 6.40 & 13.9 & 1.1 & 2749 & 1776 & 974 \\
\hline $19 \mathrm{H}-6,13 \mathrm{l}-133$ & 176.90 & 176.65 & 144.814 & 6.72 & 15.8 & 1.1 & 2771 & 1789 & 981 \\
\hline $20 \mathrm{H}-1.55-57$ & 178.06 & 178.00 & 146.030 & 7.76 & 13.2 & 1.1 & 2782 & 1796 & 986 \\
\hline $20 \mathrm{H}-1,132-134$ & 178.83 & 178.67 & 146.633 & 6.28 & 15.7 & 1.0 & 2794 & 1804 & 991 \\
\hline $20 \mathrm{H}-2,57-59$ & 179.58 & 179.42 & 147.309 & 7.10 & 12.7 & 10 & 2808 & 1812 & 996 \\
\hline $20 \mathrm{H}-2,137-139$ & 180.38 & 180.22 & 148.029 & 9.45 & 14.9 & 0.9 & 2818 & 1818 & 1001 \\
\hline $20 \mathrm{H}-3,57-59$ & 181.08 & 180.85 & 148.596 & 7.00 & 18.3 & 1.1 & 2831 & 1825 & 1006 \\
\hline $2 \mathrm{OH}-3,132-134$ & 181.83 & 181.60 & 149.272 & 7.06 & 17.6 & 1.1 & 2844 & 1833 & 1011 \\
\hline $20 \mathrm{H}-4,57-59$ & 182.58 & 182.33 & 149.929 & 7.91 & 17.3 & 10 & 2857 & 1840 & 1016 \\
\hline $20 \mathrm{H}-4,132-134$ & 183.33 & 183.09 & 150.614 & 7.08 & 17.7 & 1.1 & 2869 & 1848 & 1021 \\
\hline $20 \mathrm{H}-5,57-59$ & 184.08 & 183.83 & 151.280 & 6.86 & 14.4 & 1.1 & 2882 & 1856 & 1026 \\
\hline $20 \mathrm{H}-5,133-135$ & 184.84 & 184.59 & 151.965 & 7.31 & 12.6 & 0.9 & 2895 & 1863 & 1031 \\
\hline $20 \mathrm{H}-6,57-59$ & 185.58 & 185.38 & 152.676 & 4.82 & 27.2 & 1.7 & 2907 & 1871 & 1036 \\
\hline $20 \mathrm{H}-6,132-134$ & 186.33 & 186.09 & 153.316 & 7.18 & 16.7 & 1.0 & 2918 & 1877 & 1040 \\
\hline $20 \mathrm{H}-7,51-53$ & 187.04 & 186.75 & 153.910 & 5.73 & 8.0 & 0.8 & & & \\
\hline
\end{tabular}

Notes: MS = magnetic susceptibility ([average value of 2 discrete samples] - [background measurement]); MS* = magnetic susceptibility normalized for dry weight of sample; TS = total stress; FS = fluid stress (due to pore water and gas); $E S=$ vertical effective stress (ES = TS $-F S$ ).

that depth (i.e., the sum of the bulk density of each layer (in $\mathrm{Mg} / \mathrm{m}^{3}$; $\left.1 \mathrm{~g} / \mathrm{cm}^{3}=1 \mathrm{Mg} / \mathrm{m}^{3}\right)$ minus the pore water density $\left(1.025 \mathrm{Mg} / \mathrm{m}^{3}\right)$, times the thickness of each layer ( $\mathrm{m})$, times the acceleration of gravity $(\mathrm{g})$, where $\left.\mathrm{g}=9.807 \mathrm{~m} / \mathrm{s}^{2}\right)$.

\section{Carbonate Analyses}

Laboratory determinations of the percent calcium carbonate were made using the 242 index property samples. The inorganic carbon content of each sample was determined using a Coulometrics carbon dioxide coulometer. Freeze-dried samples weighing between 10 and $20 \mathrm{mg}$ were reacted in $2 \mathrm{~N} \mathrm{HCl}$ solution at $60^{\circ} \mathrm{C}$. Evolved carbon dioxide was titrated in a monoethanolamide solution with a coulometric indicator over a period of 5 to $20 \mathrm{~min}$, depending on the carbonate reactivity. Calibration was performed using pure calcium carbonate as a standard. The percentage of carbonate was calculated from the inorganic carbon (IC) content, assuming that all carbonate was in the form of calcite:

$$
\mathrm{CaCO}_{3}=\mathrm{IC} \times 8.332 .
$$


Table 2. Hole 893A age model used in this paper.*

\begin{tabular}{ccc}
\hline $\begin{array}{c}\text { Void-corrected } \\
\text { depth } \\
\text { (mbsf) }\end{array}$ & $\begin{array}{c}\text { Age } \\
\text { (ka) }\end{array}$ & $\begin{array}{c}\text { LSR } \\
\text { (cm/k.y.) }\end{array}$ \\
\hline 0.00 & 0.00 & 217 \\
3.62 & 1.67 & 185 \\
5.67 & 2.78 & 89 \\
8.71 & 6.19 & 167 \\
13.97 & 9.34 & 227 \\
16.90 & 10.6 & 126 \\
17.60 & 11.19 & 181 \\
18.75 & 11.82 & 142 \\
20.39 & 12.97 & 333 \\
21.94 & 13.44 & 224 \\
24.12 & 14.41 & 79 \\
25.58 & 16.27 & 203 \\
29.61 & 18.25 & 75 \\
31.52 & 20.79 & 144 \\
43.17 & 28.90 & 139 \\
73.89 & 51.00 & 119 \\
90.49 & 65.00 & 94 \\
113.89 & 90.00 & 0 \\
116.36 & 90.00 & 94 \\
140.69 & 116.00 & 103 \\
147.46 & 122.56 & 212 \\
156.87 & 127.00 & 111 \\
195.00 & 161.34 & \\
& & \\
& &
\end{tabular}

Note: *After Ingram and Kennett (this volume). $\mathrm{LSR}=$ linear sedimentation rate.

Duplicate analyses of 25 samples indicate a reproducibility of $0.7 \%$ with this method. The weight percentages of calcium carbonate determined for these samples are listed in Table 1.

\section{Age Model}

The chronology applied to Hole 893A, is based on twelve accelerator mass spectrometry (AMS) ${ }^{14} \mathrm{C}$ dates and the interpretation of oxygen isotope data (Ingram and Kennett, this volume; Kennett, this volume). Additional age information were provided by pollen analyses (Huesser, this volume). The age-depth model used in this study, is given in Table 2.

The $893 \mathrm{~A}$ age model was applied to Hole $893 \mathrm{~B}$ by matching features in the susceptibility records from each hole using "Analyseries" software for Macintosh computers (provided by L. Labeyrie)-we have matched features in Hole 893B (data plotted vs. depth) with similar features from Hole 893A (data plotted vs. age) to assign age values to the equivalent points in Hole 893B (also see Rack and Merrill, this volume).

\section{Magnetic Susceptibility Flux Calculations}

The magnetic susceptibility flux was calculated for each measurement interval in Hole 893A. This calculation involves multiplying the linear sedimentation rate (LSR in $\mathrm{cm} / \mathrm{k} . \mathrm{y}$.) determined for each interval from the Hole 893A age model (see Table 2; after Ingram and Kennett, this volume) by the dry bulk density for that interval (DBD in $\mathrm{g} / \mathrm{cm}^{3}$ ), to determine a mass accumulation rate (MAR in $\mathrm{g} / \mathrm{cm}^{2} /$ k.y.). The mass accumulation rate is multiplied by the susceptibility measurement for that interval (in SI units) to calculate the resulting magnetic susceptibility flux (SI units $\times\left[\mathrm{g} / \mathrm{cm}^{2} / \mathrm{k}\right.$.y.]).

\section{RESULTS Physical Properties}

A summary of the downhole index properties of samples from Hole 893A is shown in Figure 2; these data are tabulated and discussed in Kennett, Baldauf, et al. (1994), so they are not presented again in this paper. The bulk density plot was annotated with the
Hamilton (1976) empirical curves for diatom ooze (DO), pelagic clay (P), and terrigenous silt (T) to demonstrate that measured values fall within the downhole range of values for these lithologies. A best-fit line through the data is given for dry bulk density and porosity (Fig. 2).

Our initial assumption was that the bulk density of samples from Site 893 would generally follow Hamilton's terrigenous silt curve, except where there was substantial dilution by biogenic sources of sediment (forming skeletons of calcium carbonate and biogenic silica) or where there were increased amounts of preserved organic matter; this assumption was based on the high percentage of terrigenous material known to accumulate in this basin from previous studies (Fleischer, 1972; Thornton, 1981, 1984; among others). The observed distribution of density values seems to validate this assumption.

While it is clear that gravitational compaction exerts the major control on downhole trends in index properties, we note that finescale changes in lithology seem to greatly influence the variability of these values; low density intervals in Hole 893A generally correspond to intervals with increased concentrations of organic carbon (see Gardner and Dartnell, this volume; Stein and Rack, this volume) and siliceous microfossils (Kennett, Baldauf, et al., 1994), although siliceous data is limited to smear slide descriptions at this time (no quantitative data is available).

Below a highly gradient zone in the upper $\sim 18 \mathrm{mbsf}$, which corresponds to laminated beds of diatom nannofossil silty clay and diatom nannofossil silt with generally high water content, porosity generally decreases downhole as density increases. The major exceptions to this overall trend are related to discrete high density (low porosity) sand layers or turbidites, and an observed increased variability in porosity (density) values below $\sim 110 \mathrm{mbsf}$, where laminated intervals again become common. Bulk density values are known to be quite sensitive to the presence of increased amounts of diatom skeletons composed of low grain density amorphous silica (Hamilton, 1976; Mayer, 1979; Lee, 1982) and to the presence of increased amounts of foraminifers with high internal porosity (Bachman, 1984).

Figure 2 also contains a plot of the weight percentage of calcium carbonate in index property samples from Hole 893A. This plot reveals the overall trends in carbonate content, but these are better revealed in the much higher resolution data of both Gardner and Dartnell (this volume), and Stein and Rack (this volume). In Figure 3 , we have compared the weight percentages of calcium carbonate determined from index property samples versus measurements of magnetic susceptibility and index properties made on these same samples; the index properties of these 53 samples were measured using the Penta pycnometer and are thus considered to be of higher quality than other samples tested (see discussion in Kennett, Baldauf, et al., 1994). These data show that carbonate content is weakly correlated with index properties and even less so with magnetic susceptibility; increased carbonate content is associated with lower density and higher values of porosity and water content.

Using these same samples, we have also investigated the correlation between magnetic susceptibility and index properties (Fig. 4). Once again, these comparisons indicate only a weak correlation between index properties and magnetic susceptibility, where increased susceptibility is associated with higher density values and lower porosity and water content values. The weight percentage of total organic carbon was determined for a number of additional samples located immediately adjacent to the index property samples (data from Stein and Rack, this volume). These results were used to compare the magnetic susceptibility and organic carbon content of similar samples; these data show a weak inverse correlation, with higher susceptibilities being associated with lower percentages of total organic carbon. 

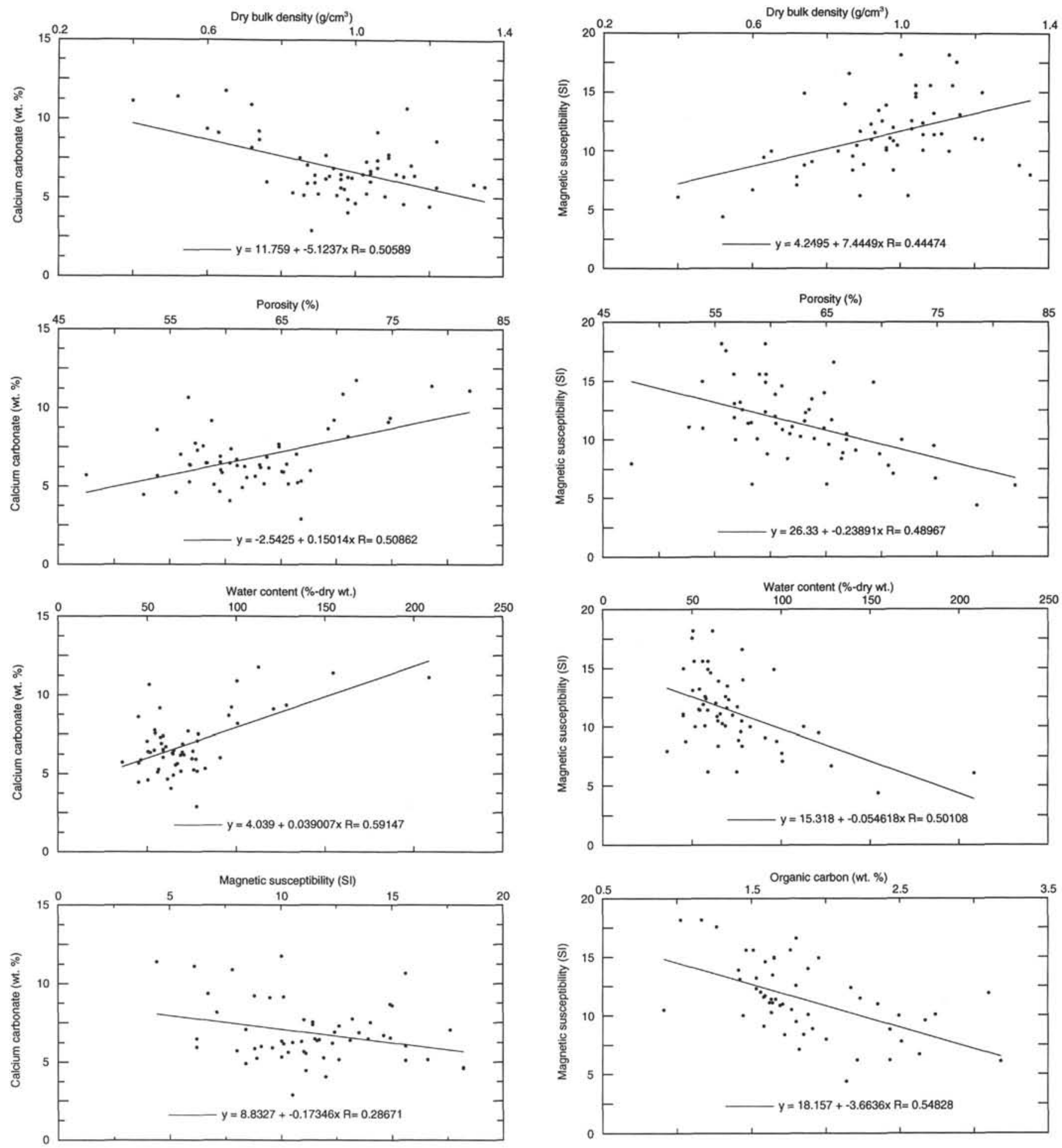

Figure 3. Scatter plots of calcium carbonate vs. dry bulk density, porosity, water content, and magnetic susceptibility, respectively, for 53 samples from Hole 893A. Linear regression equations are presented for each comparison.

\section{Shipboard vs. Shore-based Magnetic Susceptibility Records}

Because whole-core susceptibility measurements were made aboard JOIDES Resolution, but split-core measurements were made following the transport of these cores to College Station, the follow-

Figure 4. Scatter plots of magnetic susceptibility vs. dry bulk density, porosity, water content, and total organic carbon, respectively, for 53 samples from Hole 893A. Linear regression equations are presented for each comparison.

ing section of this paper provides comparisons between these records for those intervals where data from both sources are available. These comparisons are used to ensure that the shipboard MST data are representative of the properties measured onshore and also, to assess the relative degree of disturbance that might have occurred during the shipment of these cores, prior to the shore-based measurements program. 
GRAPE density $\left(\mathrm{g} / \mathrm{cm}^{3}\right) \quad$ Mag. susc. (SI - shipboard)

Mag. susc. (SI-shore)

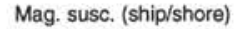

Gray bed thickness $(\mathrm{cm})$
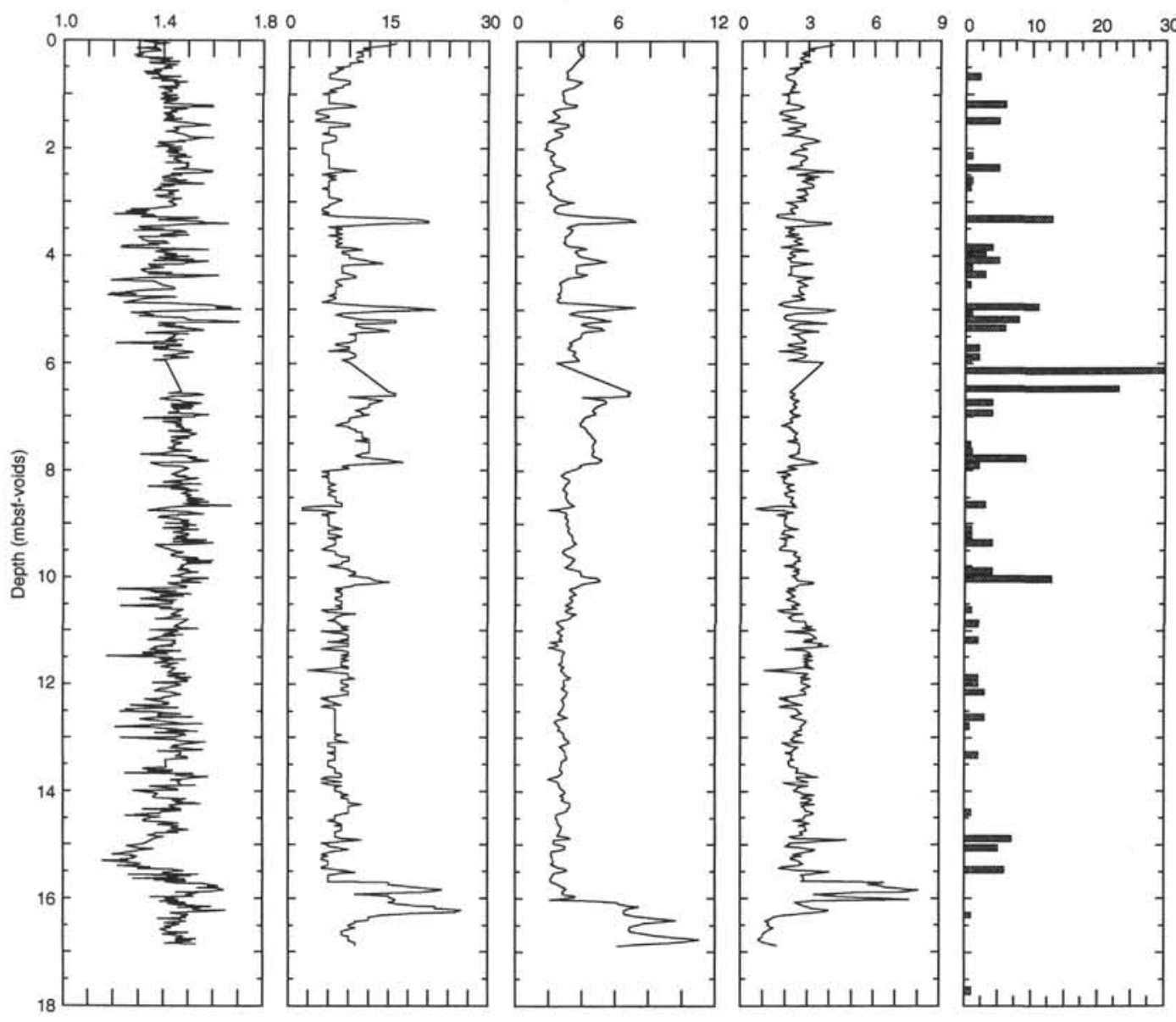

Figure 5. Comparisons between whole-core shipboard measurements (GRAPE density and magnetic susceptibility) and split-core shore-based measurements (magnetic susceptibility) from Core 146-893A-1H to Section 3H-1. The data are plotted vs. void-corrected depth (mbsf-voids). The ratio of ship/shore magnetic susceptibility measurements provides a means of identifying mismatches between these two data sets. The thickness and position of individual gray beds are also plotted.

To do this, we have plotted the shipboard MST data for each available interval (gamma-ray attenuation porosity evaluator (GRAPE) bulk density and magnetic susceptibility measurements made every $1-2 \mathrm{~cm}$ and $\sim 3 \mathrm{~cm}$, respectively) on the void-corrected depth scale developed for Hole 893A; we then compare these data with shore-based split-core susceptibility measurements from these same intervals (Figs. 5-9). The location of gray beds, sand layers, siltstone pebbles, and shell beds are shown (or noted where appropriate) in each figure. We have also calculated the ratio of shipboard to shore-based values to easily identify intervals where large discrepancies occur between the two records.

These comparisons can be quite instructive because they show: (1) how differences in the amplitude of the susceptibility signal between shipboard and shore-based data are related to differences in the sample volumes used to make whole-core or split-core measurements, and to the size of the Bartington loop used to make these measurements ( $80 \mathrm{~mm}$ vs. $100 \mathrm{~mm}$ loop); and (2) how the effects of downhole sediment compaction are revealed in the ratio between shipboard and shore-based measurements - the average value of this ratio increases with increasing depth in the hole, following the general trend of increasing bulk density with increasing depth. The increase in the ship/shore susceptibility ratio demonstrates again that there is a weak general relationship between increasing density (decreasing porosity) and higher susceptibility values, as is shown in Figure 4 .
In Figure 5, there is a large increase in susceptibility at the transition between Sections 146-893A-1H-CC and 3H-1; this transition is recorded differently by the two records, as is shown by the large increase in the ship/shore ratio at $\sim 15.5 \mathrm{mbsf}$. The observed differences are related to the lack of shore-based data from Section 146-893A$1 \mathrm{H}-\mathrm{CC}$, which results in an apparent mismatch between the records. The average ship/shore ratio is approximately equal to 2.0 within this interval; small differences in this ratio may be due to: (1) actual mismatches in the data, (2) the use of different diameter susceptibility loops, or (3) measurement noise. The transition between Cores 146$893 \mathrm{~A}-1 \mathrm{H}$ and $2 \mathrm{H}$ is clearly seen as a straight line in the records at $\sim 6$ mbsf.

Core 146-893A-5H (Fig. 6) exhibits a large number of gas-related voids that serve to degrade the quality of the MST data within this core. Even after removing most of the voids from the records, their influence is still observed, although at least some of the lowered density and susceptibility values may be caused by the presence of increased amounts of biogenic sediment in this core. The average ratio of ship/shore measurements is $\sim 3$, slightly higher than was observed in Figure 5.

The magnetic susceptibility data from Sections 146-893A-10H-3 through $10 \mathrm{H}-5$, exhibit a large number of relative peaks overlain on a general increase in background values (Fig. 7); these peaks seem to be related to the presence of small siltstone pebbles and thin sand layers. A 13-cm-thick gray layer in Section 146-893A-10H-6, 47-60 cm 


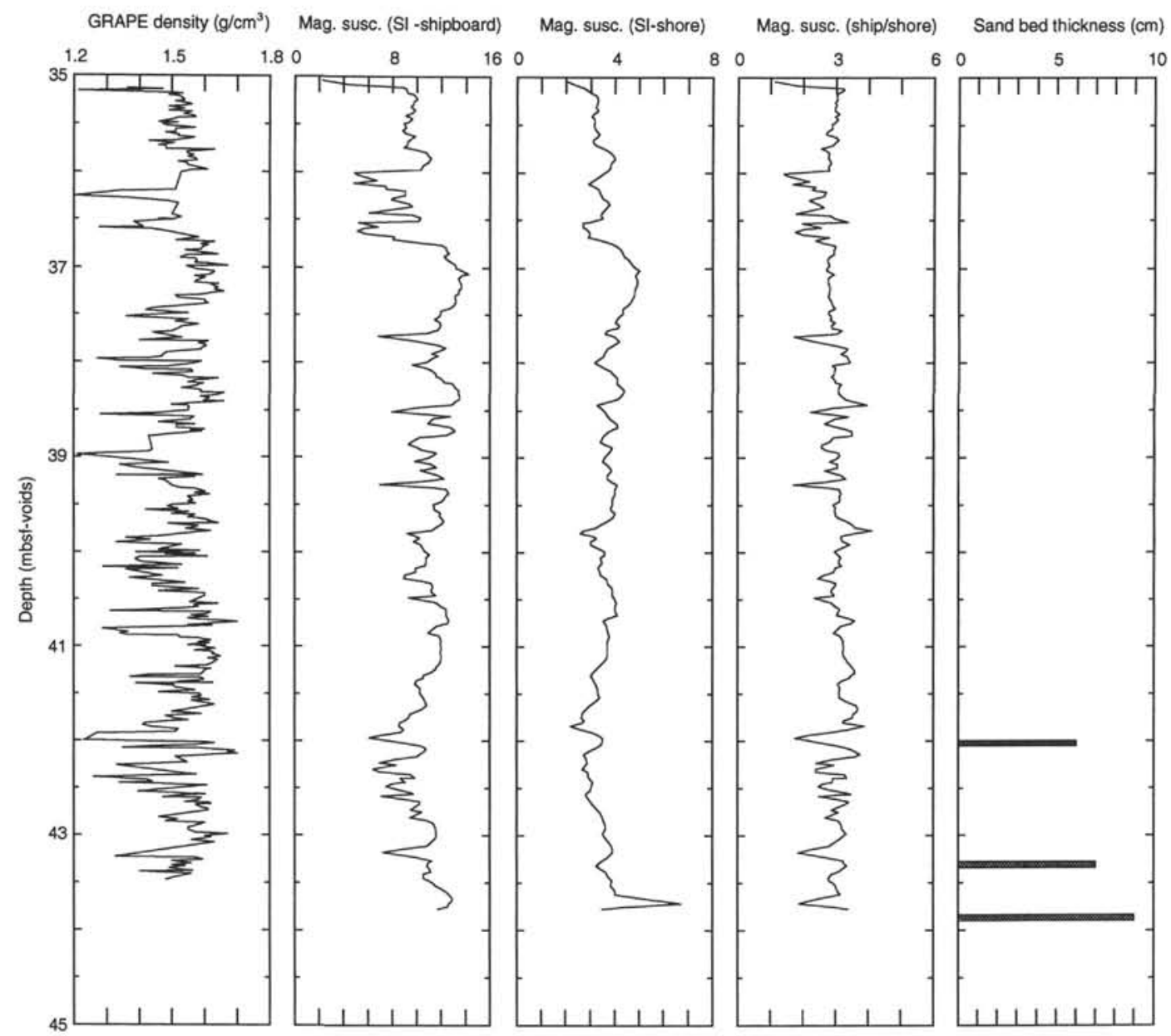

Figure 6. Comparisons between whole-core shipboard measurements (GRAPE density and magnetic susceptibility) and split-core shore-based measurements (magnetic susceptibility) made on Core 146-893A-5H. The thickness and position of individual sand layers are also plotted.

( $90.4 \mathrm{mbsf})$, is clearly observed as a peak in both data sets. The largest mismatch seen in the ship/shore ratio occurs at $\sim 86.4 \mathrm{mbsf}$, where a peak appears to be missing from the shore-based measurements (in Section 146-893A-10H-3).

The data from Core 146-893A-15H (Fig. 8) reflect the presence of a relatively dense, nonlaminated interval of silty clay extending from the middle of Section 146-893A-15H-4 to the middle of Section 146$893 \mathrm{~A}-15 \mathrm{H}-5$. Magnetic susceptibility and GRAPE density values increase within this interval. There seems to be a fair amount of mismatch in ship/shore magnetic susceptibility values below Section 146-893A-15H-6 (>137.5 mbsf); two susceptibility peaks attributed to gray layers are shifted upward in the shipboard data, possibly indicating some disturbance of this core during core transport or core splitting operations.

The dominant feature of the data collected from Core 146-893A$20 \mathrm{H}$ (Fig. 9) is the presence of a large increase in magnetic susceptibility in the middle of Section 146-893A-20H-6, which is related to the presence of two gray, silty clay beds at $40-45 \mathrm{~cm}$ and $64-73 \mathrm{~cm}$, respectively, with a shell bed located between them (Kennett, Baldauf, et al., 1994). The average ship/shore ratio in this core has increased to $\sim 3.5$, from $\sim 2.0$ in Figure 5 ; the average bulk density increased from $\sim 1.5 \mathrm{~g} / \mathrm{cm}^{3}$ to $1.8 \mathrm{~g} / \mathrm{cm}^{3}$ (porosity values decreased from $\sim 80 \%$ to $55 \%$ ) over this same interval because of the effects of gravitational compaction on the sediment column.

\section{Magnetic Susceptibility}

Magnetic susceptibility is generally low in biogenic sediments, which are primarily a collection of microfossil skeletons composed of amorphous opal (e.g., diatoms and radiolarians) or calcite (e.g., foraminifers and coccoliths) and is generally high in terrigenous materials, which have higher concentrations of magnetic minerals (e.g., magnetite; Thompson and Oldfield, 1986). Exceptions to this general characterization (e.g., high susceptibility in terrigenous sediments) often occur in marine sediments because of: (1) increasing distance from relevant sources of magnetic minerals, (2) dilution by other sedimentary components, (3) diagenetic reactions (reductive diagenesis), and/or (4) the production of biogenic magnetite, among other reasons.

The magnetic susceptibility data from Hole 893 A show that distinctive zones having increased susceptibility values are separated by zones with low values of susceptibility (see Fig. 2). A comparison is made between a 10-point smoothed version of the Hole 893A susceptibility data and plots which show the relative positions of gray beds and sand layers in this hole (Fig. 10). Distinct layers of gray, silty clay to clayey silt are observed throughout much of the laminated intervals in the upper portions of both holes (see Figs. 5 and 10). Many of these gray layers are associated with increases in GRAPE bulk density and with increases in magnetic susceptibility. We suggest that the ob- 


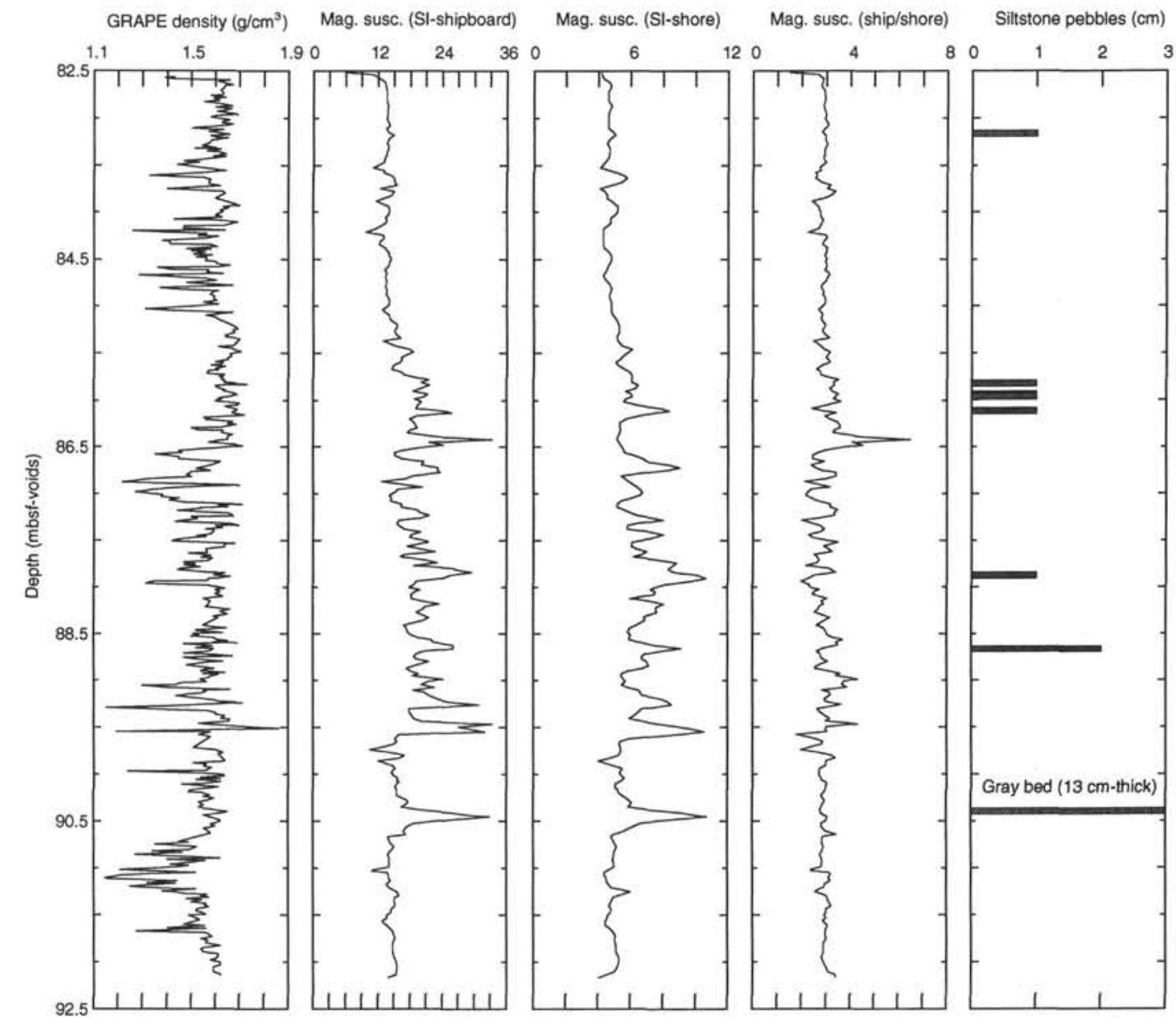

Figure 7. Comparisons between whole-core shipboard measurements (GRAPE density and magnetic susceptibility) and split-core shore-based measurements (magnetic susceptibility) made on Core 146-893A-10H. The position and number of individual siltstone pebbles are also plotted; note the gray bed near 90.5 mbsf.

served increases in the susceptibility of the gray beds are caused by greater amounts of magnetic minerals in these layers, although this observation has not yet been confirmed in the laboratory.

The void-corrected depths and estimated thicknesses of gray beds, massive intervals, and sand layers in the deeper portions of Holes $893 \mathrm{~A}$ and $893 \mathrm{~B}$ are given in Tables 3 and 4 , as compiled from published core descriptions (Kennett, Baldauf, et al., 1994) and from examinations of core photographs. A listing of these features in the upper portion of these two holes is given in Rack and Merrill (this volume), who provide a more detailed discussion of the past 16,000 yr of gray bed ("event") deposition.

We observe seven distinct zones with generally higher susceptibility values relative to the background values measured in Hole $893 \mathrm{~A}$, although this list is by no means conclusive (see Fig. 10). These zones are located (1) from $\sim 3$ to $8 \mathrm{mbsf}$ (approximate age $=1.4$ to $5.2 \mathrm{ka}$ ); (2) from $\sim 16$ to 20 mbsf ( 10.2 to $13 \mathrm{ka}$ ); (3) from $\sim 52$ to 64 mbsf ( $\sim 35$ to $\sim 44 \mathrm{ka}$ ), with additional sharp increases at $\sim 65.5$ mbsf and $\sim 67.7$ mbsf; (4) from $\sim 81$ to $\sim 92$ mbsf ( $~ 57$ to $\sim 67 \mathrm{ka}$ ); (5) intermittently from 102 to 118 mbsf ( $\sim 77$ to $\sim 92 \mathrm{ka}$ ); (6) from $\sim 135$ to $\sim 137$ mbsf $(\sim 110$ to $\sim 112 \mathrm{ka}$ ); and (7) from $\sim 144$ mbsf (>119 ka) to the base of the hole, although there are also some relatively sharp discrete peaks in this deeper zone.

The Hole 893A magnetic susceptibility data is compared with plots of the weight percentages of calcium carbonate and total organ- ic carbon (TOC) determined at $\sim 20$-cm sample spacing (Stein and Rack, this volume) in Figure 11. A comparison of these plots clearly shows the apparent relationship between TOC and magnetic susceptibility. Several intervals show both higher percentages of total organic carbon and lower susceptibility values (i.e., $\sim 45-55 \mathrm{ka}, \sim 70-80$ $\mathrm{ka}, \sim 95-105 \mathrm{ka}$, and $\sim 112-125 \mathrm{ka}$ ); numerous intervals are also observed with the opposite relationship (i.e., having both lower weight percentages of TOC and higher values of magnetic susceptibility). Large increases in TOC are generally associated with the preservation of intervals of laminated sediment during the warm interstadials $5 \mathrm{e}, 5 \mathrm{c}$, and $5 \mathrm{a}$, and during the Holocene (Stein and Rack, this volume).

The generally reduced background susceptibility values within these intervals may reflect changes caused by reductive diagenesis in association with suboxic or anoxic bottom waters in the SBB at these times. However, susceptibility values still increase in the presence of gray beds within these intervals, suggesting that the event deposits may reflect a primary detrital signal.

There does not seem to be much of a relationship between magnetic susceptibility and the weight percentage of calcium carbonate, although some intervals with lower carbonate values are associated with increases in total organic carbon (Fig. 11). Similar carbonate and organic carbon data are available from sample intervals offset by 10 $\mathrm{cm}$ from the data given in Stein and Rack (this volume) (see Gardner 


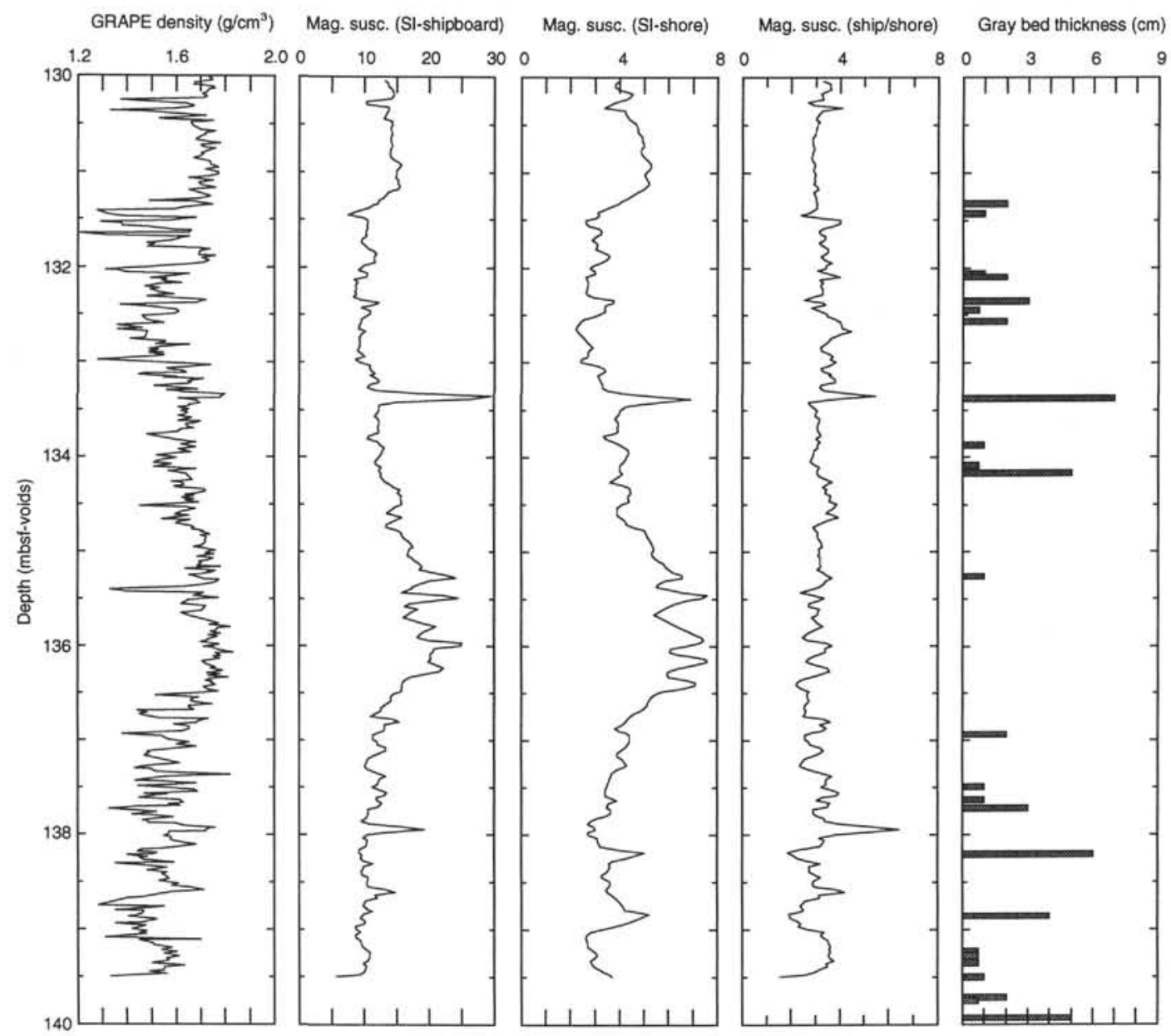

Figure 8. Comparisons between whole-core shipboard measurements (GRAPE density and magnetic susceptibility) and split-core shore-based measurements (magnetic susceptibility) made on Core 146-893A-15H. The thickness and position of individual gray beds are also plotted.

and Dartnell, this volume). When these two data sets are combined, they will provide a $10-\mathrm{cm}$ sample-spacing over the entire length of core in Hole 893A.

\section{Correlation Between Holes 893A and 893B}

One of the objectives of this study was to use the magnetic susceptibility data to correlate between the cores collected from Holes $893 \mathrm{~A}$ and $893 \mathrm{~B}$. We have used "AnalySeries" software to correlate between susceptibility peaks in each hole and to transfer the Hole $893 \mathrm{~A}$ age model to Hole 893B (Fig. 12). We have identified 39 tiepoints between the magnetic susceptibility data from these two holes and have calculated the relative offsets based on these correlations (Table 5). The overall correlation coefficient for the match between these two $\sim 70$-m-long records is 0.614 . The match between these two records is more speculative in the central portion from 15 to 30 k.y. because of (1) the lack of distinctive features in the records, (2) missing (nonrecovered) intervals of sediment from one or the other hole, and (3) because of the gas expansion in the cores. Rack and Merrill (this volume) provide a detailed summary of the interhole correlations from the seafloor to approximately $24 \mathrm{mbsf}$ ( 0 to $16,000 \mathrm{yr}$ ago).

\section{Magnetic Susceptibility Flux}

Magnetic susceptibility flux values were calculated for each measurement interval in Hole $893 \mathrm{~A}$, as previously explained in the
"Methods" section of this paper (Fig. 13). The shape of the susceptibility flux profiles are very similar for the various measurements of magnetic susceptibility discussed in this paper (discrete measurements, normalized ratios, and closely spaced measurements). These profiles are significantly influenced by the age model, because large changes in the linear sedimentation rate (i.e., the increases shown at $\sim 18$ k.y. and $\sim 125$ k.y.) can cause significant changes in mass accumulation rates, which may or may not reflect a natural change in sediment accumulation.

In Figure 13, the increases in magnetic susceptibility flux (MS flux) generally occur within the same intervals that were previously noted as being associated with higher susceptibility values (see discussion of Fig. 10). These intervals generally correspond to glacial stages having heavier oxygen isotope values (e.g., colder intervals); we note that some of the largest MS-flux increases occur at the transitions between isotope stages.

One of the most prominent increases in the MS-flux record from Hole $893 \mathrm{~A}$ occurs between 10 and $13 \mathrm{ka}$, which is approximately equivalent to the Bølling-Allerød through Younger Dryas period (Berger, 1990). Other significant features of the MS-flux record include the intermittent fluctuations (increased/decreased flux) throughout glacial Stages 2 and 3, and the broad increase in MS flux centered on glacial Stage 4. The large peak in apparent MS flux centered on $\sim 125$ k.y. (i.e., the transition to isotope sub-Stage $5 \mathrm{e}$ from glacial Stage 6), may be exaggerated by a large increase in linear sedimentation rate across this interval, as previously noted. 


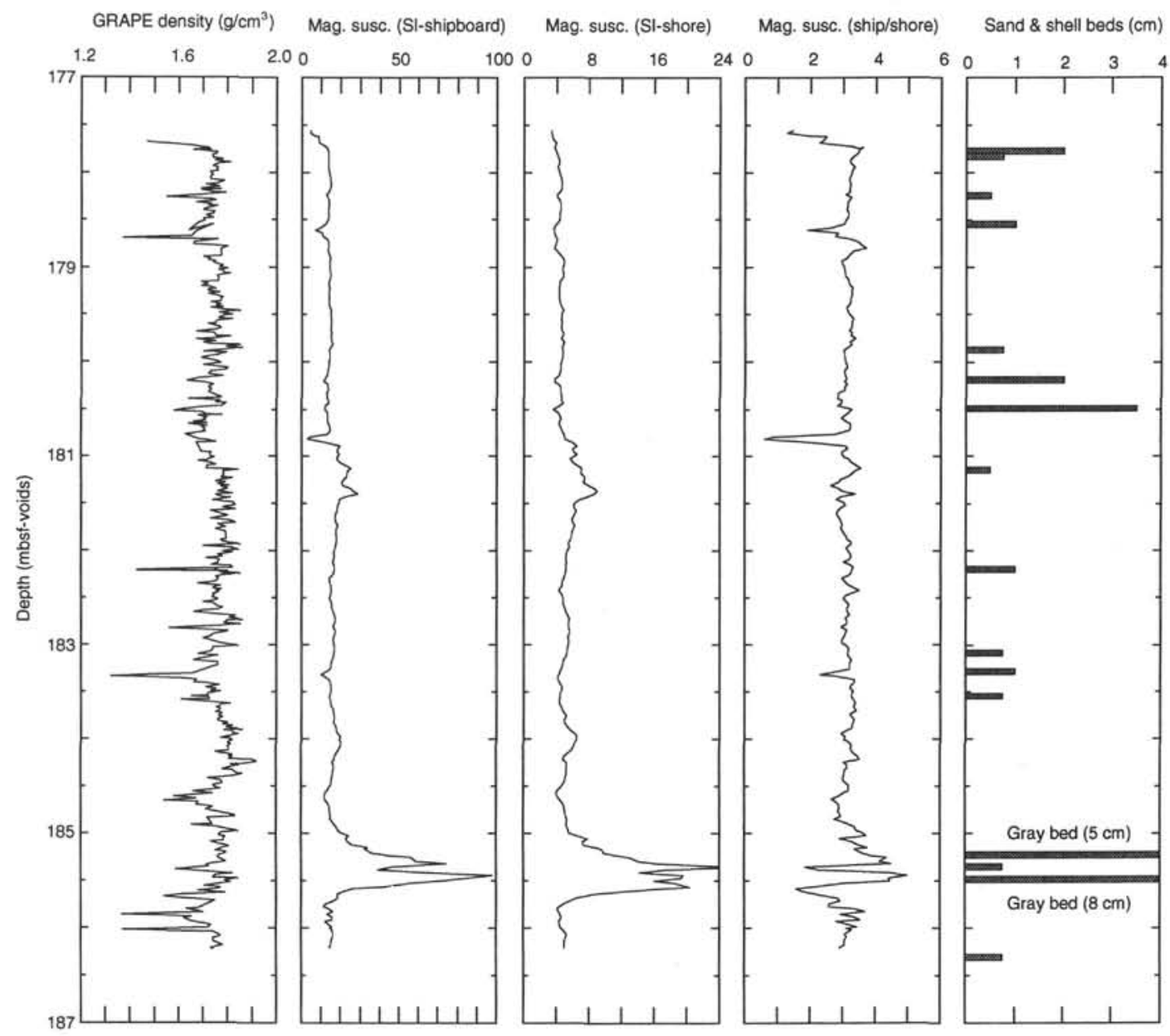

Figure 9. Comparisons between whole-core shipboard measurements (GRAPE density and magnetic susceptibility) and split-core shore-based measurements (magnetic susceptibility) made on Core 146-893A-20H. The thickness and position of individual sand layers or shell beds are also plotted; note the two gray layers associated with the large peak(s) in susceptibility.

\section{DISCUSSION}

\section{Introduction}

The primary controls on the deposition and preservation of sedimentary layers in the Santa Barbara Basin are (1) changes in relative sea level (through changes in basin geometry, ocean circulation patterns, and sediment transport paths across/along the shelf and slope); (2) changes in atmospheric and/or oceanographic variables (through relative changes in upwelling intensity and productivity, changes in atmospheric circulation and precipitation patterns; or through changes in the rates of geochemical and/or diagenetic processes in the ba$\sin$ ); and (3) changes in the rate or direction of tectonic motions (e.g., convergence across the Santa Barbara Channel and uplift of the Transverse and Coast Ranges, with resulting changes in stream profiles, drainage patterns, and the frequency of earthquakes).

We contend that the major trends in the Hole 893A magnetic susceptibility record are the result of significant variations in the influx of terrigenous (detrital, magnetic) material to the Santa Barbara Basin during glacial times and during climatic transitions. We recognize that geochemical reactions and possible magnetic mineral diagenesis are surely important in those portions of the record, which are associated with higher sea level, low oxygen levels in the deeper parts of the basin, and the preservation of sedimentary laminae; these intervals are also generally associated with lowered susceptibility values and higher percentages of total organic carbon.
The first order, long-term ( $>50,000 \mathrm{yr}$ ) changes in terrigenous sediment accumulation in the Santa Barbara Basin are likely controlled by changes in sea level caused by fluctuations in global ice volume (see Fig. 14). Changes in relative sea level, caused by fluctuations (growth and decay) in the size (mass) of large continental ice sheets, would change the size and shape of the Santa Barbara Basin and the sediment budget of the basin (Schwalbach and Gorsline, 1985). The profiles of magnetic susceptibility, MS flux, and total organic carbon vs. age (Fig. 14), are quite similar in character to the shape of the ice mass fluctuations calculated from oxygen isotope values (Imbrie et al., 1984; Saltzman and Verbitsky, 1994). Intervals having increased ice mass (lower sea levels) are generally coincident with intervals having higher values of magnetic susceptibility and MS flux and lower values of total organic carbon in the SBB; conversely, intervals of lowered ice mass (higher sea levels) are generally coincident with intervals having lower values of magnetic susceptibility and MS flux and higher values of total organic carbon in the SBB (Fig. 14).

In the following paragraphs, we will discuss the various working hypotheses used to evaluate the significance of the magnetic susceptibility record at Site 893 , in terms of our present understanding of (1) changes in mean sea level and the probable effect of these fluctuations on the evolution of Quaternary landscapes; (2) tectonic motions in the Santa Barbara region, including historical records of earthquake activity, recent geodetic measurements of deformation, and the possible effects that these motions might have on slope instability; 


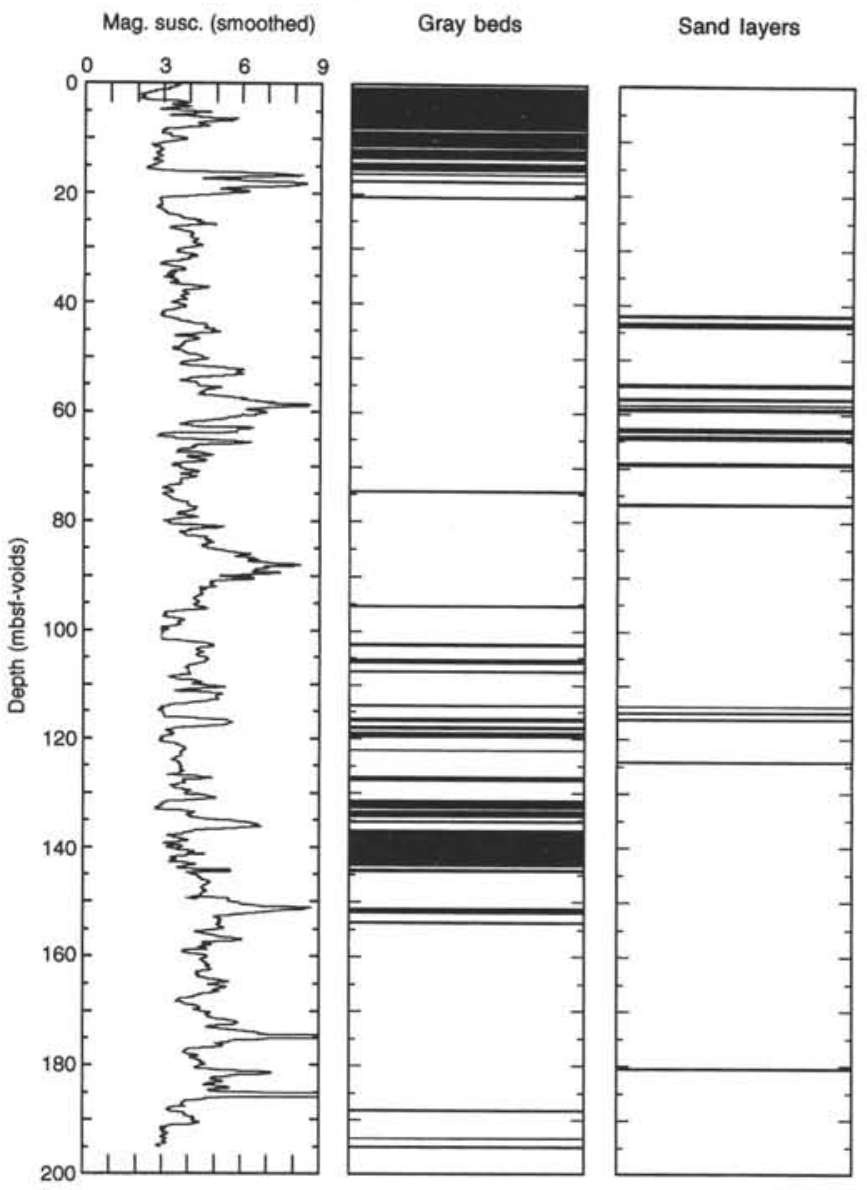

Figure 10. Comparison between 10-point smoothed magnetic susceptibility data from Hole 893A and the position of gray beds and sand layers plotted vs. void-corrected depth. The depths of gray bed and sand layers are given in Table 3 (also see table 2 in Rack and Merrill, this volume). Gray beds are best preserved within laminated intervals of Hole 893A.

and (3) changes in climatic factors, including a brief assessment of past paleoceanographic and palynological studies and a discussion of possible atmospheric connections between the record at Site 893 and other paleoclimatic records and models.

\section{Sea-level Fluctuations}

Numerous studies have evaluated the quality and chronology of various estimates of sea level for the late Quaternary (Gallup et al., 1994; Peltier, 1994; Tushingham and Peltier, 1991; among others) with many of these studies being specifically focused on the interval since the Last Glacial Maximum or on the Younger Dryas event (Bard et al., 1989, 1990; Edwards et al., 1993; Fairbanks, 1989; among others). The predicted or measured changes in relative or mean sea level are intimately related to the rate and timing of changes in global ice volume and to the interaction of ice sheets with the ocean and solid earth (Peltier, 1994; Tushingham and Peltier, 1991) through changes in paleotopography.

During the Last Glacial Maximum, sea level was approximately $\sim 121 \pm 5 \mathrm{~m}$ below present level (Fairbanks, 1989), as reflected in the predicted seaward expansion of land areas across the shelf of the Santa Barbara Basin (shown in Fig. 1B). During the Eemian period (isotope Stage 5e), sea level was approximately 2 to $8 \mathrm{~m}$ higher than at present (Gallup et al., 1994). During other periods in the past 160,000 years, sea levels were at intermediate levels relative to these extreme values; the timing and relative magnitude of these intermediate sealevel fluctuations are generally deduced from oxygen isotope records (Imbrie et al., 1984).

Holocene and Eemian periods of relatively high sea levels are associated with the preservation of laminated intervals in Hole 893B and well-preserved gray layers, especially after $\sim 8 \mathrm{ka}$. A deceleration in the rate of sea-level rise was suggested by Stanley and Warne (1994) for the interval from about 8.5 to 6.5 k.y., based on studies of globally distributed Holocene deltaic sequences. This interval corresponds to low susceptibility values at Site 893 and increasing preservation of laminated sediments in the central Santa Barbara Basin.

\section{Tectonic Motions, Earthquakes, and Slope Stability}

Tectonic motions in the Santa Barbara and Ventura basins were the subject of several recent studies designed to measure convergence across these basins. These studies have used both geological information (Yeats, 1983; Shaw and Suppe, 1994) and geodetic measurements of deformation (Donnellan et al., 1993; Larson, 1993; Larson and Webb, 1992) to determine convergence rates across different regions of these basins.

Geodetic measurements have indicated that motions in the Santa Barbara Channel are consistent with northeast-southwest compressional deformation rates of about $6 \mathrm{~mm} / \mathrm{yr}$ in the eastern channel, with lower rates of deformation $(\sim 2.3 \mathrm{~mm} / \mathrm{yr})$ in the western channel (Larson, 1993; Larson and Webb, 1992); geologically determined convergence rates are generally larger (Yeats, 1983). Seismic and geodetic studies have shown that seismicity in the eastern region of the Santa Barbara Channel is significant (Larson, 1993; Lee and Vedder, 1973). A recent study of active faulting and growth folding in the eastern Santa Barbara Channel suggests that significant earthquakes may occur along blind thrust faults in this eastern region with a recurrence interval of $\sim 1500 \mathrm{yr}$ (Shaw and Suppe, 1994). It may be possible to identify particular features in the sediment record at Site 893, which might correspond to earthquake-initiated mass movements of sediment.

While it is well accepted that many of the gray layers and massive beds that are preserved in the Santa Barbara Basin (and at Site 893) have a common origin as flood deposits, some of the thicker gray layers and/or massive intervals were attributed to fine-grained turbidites having origins on the middle or upper slope (Thornton, 1984, 1986). The sediment stability of these slope deposits is affected by many factors, including sediment shear strength, consolidation state, sedimentation rates, organic carbon content, and triggering mechanisms, among others (Field and Edwards, 1980; Gorsline et al., 1984; Prior and Colman, 1984; Thornton, 1984).

Earthquake accelerations can initiate sediment instability through increases in sediment pore pressures and inertial forces under the cyclic-loading conditions associated with earthquake shaking (Busch and Keller, 1983). Pore water and pore gas pressures can be high in these continental margin settings (the Site 893 cores expanded greatly because of elevated levels of pore gas; Kennett, Baldauf, et al., 1994); these pressures can approach geostatic values in particular locations. The effect of lowered vertical effective stresses by excess pore water or gas pressures can result in slope failures even on low angle slopes $\left(<1^{\circ}\right.$; Prior and Coleman, 1984).

Sediment behavior is also significantly influenced by the relative concentrations of organic matter in these deposits (Keller, 1982; Busch and Keller, 1982, 1983). Organic matter absorbs water and causes clay-sized particles to aggregate, thus, forming an open fabric (Keller, 1982); this open fabric results in high water contents and increased plasticity, and low bulk densities (Booth and Dahl, 1986; Pusch, 1973). The high water content and deformability of organiccarbon rich sediment can contribute to the overall ductile behavior of the sediment, but gravitational forces alone are often insufficient to cause sediment failure on the slope (Lee and Edwards, 1986). 
Table 3. Gray beds, massive beds, and sand layers in Hole 893A.

\begin{tabular}{|c|c|c|c|c|c|}
\hline \multirow{2}{*}{$\begin{array}{l}\text { Core, section, } \\
\text { interval }(\mathrm{cm})\end{array}$} & \multicolumn{3}{|c|}{ Void-corrected depth (mbsf) } & \multirow{2}{*}{$\begin{array}{l}\text { Layer } \\
\text { thickness } \\
(\mathrm{cm})\end{array}$} & \multirow[b]{2}{*}{ Type } \\
\hline & Top & Bottom & Midpoint & & \\
\hline \multicolumn{6}{|l|}{$146-893 \mathrm{~A}-$} \\
\hline $5 \mathrm{H}-6,13-19$ & 41.99 & 42.05 & 42.02 & 6.00 & $\mathrm{~S}$ \\
\hline $5 \mathrm{H}-7,20-27$ & 43.27 & 43.34 & 43.31 & 7.00 & $\mathrm{~S}$ \\
\hline $5 \mathrm{H}-\mathrm{CC}, 16-25$ & 43.82 & 43.91 & 43.86 & 9.00 & $\mathrm{~S}$ \\
\hline $7 \mathrm{H}-2,105-150$ & 54.50 & 54.95 & 54.72 & 45.00 & $\mathrm{~S}$ \\
\hline $7 \mathrm{H}-4,118-135^{*}$ & 57.11 & 57.28 & 57.19 & 17.00 & $\mathrm{~S}$ \\
\hline $7 \mathrm{H}-5,80-150$ & 58.36 & 59.06 & 58.71 & 70.00 & $\mathrm{~S}$ \\
\hline $7 \mathrm{H}-6,0-30$ & 59.03 & 59.33 & 59.18 & 30.00 & $S$ \\
\hline $7 \mathrm{H}-8,75-90$ & 62.66 & 62.81 & 62.74 & 15.00 & $\mathrm{~S}$ \\
\hline $7 \mathrm{H}-\mathrm{CC}, 25-4 \mathrm{I}$ & 63.04 & 63.20 & 63.12 & 16.00 & $\mathrm{~S}$ \\
\hline $8 \mathrm{H}-2,43-95$ & 64.08 & 64.60 & 64.34 & 52.00 & $\mathrm{~S}$ \\
\hline $8 \mathrm{H}-6,27-52$ & 68.95 & 69.20 & 69.07 & 25.00 & $\mathrm{~S}$ \\
\hline $8 \mathrm{H}-6,57-70$ & 69.25 & 69.38 & 69.32 & 13.00 & $\mathrm{~S}$ \\
\hline $9 \mathrm{H}-2,21-29$ & 74.46 & 74.54 & 74.50 & 8.00 & GB \\
\hline $9 \mathrm{H}-3,130-139$ & 76.60 & 76.69 & 76.64 & 9.00 & $\mathrm{~S}$ \\
\hline $11 \mathrm{H}-3,49-53$ & 95.38 & 95.42 & 95.40 & 4.00 & GB \\
\hline $12 \mathrm{H}-1,111-113$ & 102.50 & 102.52 & 102.51 & 2.00 & GB \\
\hline $12 \mathrm{H}-1,127-130$ & 102.60 & 102.63 & 102.61 & 3.00 & GB \\
\hline $12 \mathrm{H}-3,111-112$ & 105.37 & 105.38 & 105.38 & 1.00 & GB \\
\hline $12 \mathrm{H}-3,148-150$ & 105.74 & 105.76 & 105.75 & 2.00 & GB \\
\hline $12 \mathrm{H}-4,0-7$ & 105.76 & 105.83 & 105.79 & 7.00 & GB \\
\hline $12 \mathrm{H}-5,40-42$ & 107.57 & 107.59 & 107.58 & 2.00 & GB \\
\hline $12 \mathrm{H}-8,0-13$ & 109.34 & 109.47 & 109.41 & 13.00 & GB \\
\hline $13 \mathrm{H}-1,64-67$ & 111.34 & 111.37 & 111.36 & 3.00 & S \\
\hline $13 \mathrm{H}-4,24-30$ & 113.84 & 113.90 & 113.87 & 6.00 & GB \\
\hline $13 \mathrm{H}-4,30-150$ & 113.89 & 115.09 & 114.49 & 120.00 & $\mathrm{~S}$ \\
\hline $13 \mathrm{H}-5,0-125$ & 115.09 & 116.34 & 115.71 & 125.00 & S \\
\hline $13 \mathrm{H}-5,126-150$ & 116.35 & 116.59 & 116.47 & 24.00 & GB \\
\hline $13 \mathrm{H}-6,130-130$ & 117.89 & 117.90 & 117.89 & 0.75 & GB \\
\hline $13 \mathrm{H}-6,138-140$ & 117.97 & 117.99 & 117.98 & 2.00 & GB \\
\hline $13 \mathrm{H}-7,79-82$ & 118.89 & 118.92 & 118.90 & 3.00 & GB \\
\hline $13 \mathrm{H}-7,85-86$ & 118.95 & 118.96 & 118.96 & 1.00 & GB \\
\hline $13 \mathrm{H}-7,92-94$ & 119.02 & 119.04 & 119.03 & 2.00 & GB \\
\hline $13 \mathrm{H}-8,2-3$ & 119.62 & 119.63 & 119.63 & 1.00 & GB \\
\hline $14 \mathrm{H}-2,7-10$ & 122.09 & 122.12 & 122.10 & 3.00 & GB \\
\hline $14 \mathrm{H}-3,60-69$ & 124.16 & 124.25 & 124.21 & 9.00 & $\mathrm{~S}$ \\
\hline $14 \mathrm{H}-7,13-26$ & 127.06 & 127.19 & 127.13 & 13.00 & GB \\
\hline $14 \mathrm{H}-7,35-37$ & 127.28 & 127.30 & 127.29 & 2.00 & GB \\
\hline $14 \mathrm{H}-7,58-61$ & 127.51 & 127.54 & 127.53 & 3,00 & GB \\
\hline $15 \mathrm{H}-2,10-12$ & 131.31 & 131.33 & 131.32 & 2.00 & GB \\
\hline $15 \mathrm{H}-2,21-22$ & 131.42 & 131.43 & 131.42 & 1.00 & GB \\
\hline $15 \mathrm{H}-2,86-87$ & 132.05 & 132.06 & 132.05 & 1.00 & GB \\
\hline $15 \mathrm{H}-2,89-91$ & 132.08 & 132.10 & 132.09 & 2.00 & GB \\
\hline $15 \mathrm{H}-2,114-117$ & 132.33 & 132.36 & 132.35 & 3.00 & GB \\
\hline $15 \mathrm{H}-2,125-125$ & 132.44 & 132.45 & 132.44 & 0.75 & GB \\
\hline $15 \mathrm{H}-2,136-138$ & 132.55 & 132.57 & 132.56 & 2.00 & $\mathrm{~GB}$ \\
\hline $15 \mathrm{H}-3,6 \mathrm{l}-68$ & 133.34 & 133.41 & 133.38 & 7.00 & GB \\
\hline $15 \mathrm{H}-3,112-113$ & 133.85 & 133.86 & 133.86 & 1.00 & GB \\
\hline $15 \mathrm{H}-3,135-135$ & 134.08 & 134.09 & 134.08 & 0.75 & GB \\
\hline $15 \mathrm{H}-3,141-150$ & 134.14 & 134.19 & 134.17 & 5.00 & GB \\
\hline $15 \mathrm{H}-4,104-105$ & 135.25 & 135.26 & 135.26 & 1.00 & GB \\
\hline $15 \mathrm{H}-5,105-107$ & 136.92 & 136.94 & 136.93 & 2.00 & GB \\
\hline $15 \mathrm{H}-6,0-1$ & 137.48 & 137.49 & 137.48 & 1.00 & GB \\
\hline
\end{tabular}

\begin{tabular}{|c|c|c|c|c|c|}
\hline \multirow{2}{*}{$\begin{array}{l}\text { Core, section, } \\
\text { interval }(\mathrm{cm})\end{array}$} & \multicolumn{3}{|c|}{ Void-corrected depth (mbsf) } & \multirow{2}{*}{$\begin{array}{l}\text { Layer } \\
\text { thickness } \\
(\mathrm{cm})\end{array}$} & \multirow[b]{2}{*}{ Type } \\
\hline & Top & Bottom & Midpoint & & \\
\hline $15 \mathrm{H}-6,14-15$ & 137.62 & 137.63 & 137.63 & 1.00 & GB \\
\hline $15 \mathrm{H}-6,22-25$ & 137.70 & 137.73 & 137.71 & 3.00 & GB \\
\hline $15 \mathrm{H}-6,69-75$ & 138.17 & 138.23 & 138.20 & 6.00 & GB \\
\hline $15 \mathrm{H}-6,135-139$ & 138.83 & 138.87 & 138.85 & 4.00 & GB \\
\hline $15 \mathrm{H}-7,25-25$ & 139.22 & 139.23 & 139.22 & 0.75 & GB \\
\hline $15 \mathrm{H}-7,29-29$ & 139.26 & 139.27 & 139.26 & 0.75 & GB \\
\hline $15 \mathrm{H}-7,37-37$ & 139.34 & 139.35 & 139.34 & 0.75 & GB \\
\hline $15 \mathrm{H}-7,52-53$ & 139.49 & 139.50 & 139.49 & 1.00 & GB \\
\hline $15 \mathrm{H}-7,73-75$ & 139.70 & 139.72 & 139.71 & 2.00 & GB \\
\hline $15 \mathrm{H}-\mathrm{CC}, 15-20$ & 139.90 & 139.95 & 139.92 & 5.00 & GB \\
\hline $\mid 6 \mathrm{H}-1,31-31$ & 139.74 & 139.75 & 139.74 & 0.75 & GB \\
\hline $16 \mathrm{H}-1,70-72$ & 140.05 & 140.07 & 140.06 & 2.00 & GB \\
\hline $16 \mathrm{H}-1,75-76$ & 140.10 & 140.11 & 140.11 & 1.00 & GB \\
\hline $16 \mathrm{H}-1,97-101$ & 140.23 & 140.27 & 140.25 & 4.00 & $\mathrm{~GB}$ \\
\hline $16 \mathrm{H}-1,105-107$ & 140.31 & 140.33 & 140.32 & 2.00 & GB \\
\hline $16 \mathrm{H}-1,118-124$ & 140.41 & 140.47 & 140.44 & 6.00 & GB \\
\hline $16 \mathrm{H}-1,142-145$ & 140.59 & 140.62 & 140.60 & 3.00 & GB \\
\hline $16 \mathrm{H}-2,0-7$ & 140.69 & 140.76 & 140.73 & 7.00 & GB \\
\hline $16 \mathrm{H}-2,23-35$ & 140.92 & 141.04 & 140.98 & 12.00 & GB \\
\hline $16 \mathrm{H}-2,38-40$ & 141.07 & 141.09 & 141.08 & 2.00 & GB \\
\hline $16 \mathrm{H}-2,44-44$ & 141.13 & 141.14 & 141.14 & 1.00 & GB \\
\hline $16 \mathrm{H}-2,54-56$ & 141.25 & 141.27 & 141.26 & 2.00 & GB \\
\hline $16 \mathrm{H}-2,58-60$ & 141.27 & 141.29 & 141.28 & 2.00 & GB \\
\hline $16 \mathrm{H}-2,64-67$ & 141.33 & 141.36 & 141.35 & 3.00 & GB \\
\hline $16 \mathrm{H}-2,67-81$ & 141.35 & 141.49 & 141.42 & 14.00 & GB \\
\hline $16 \mathrm{H}-2,96-98$ & 141.65 & 141.67 & 141.66 & 2.00 & GB \\
\hline $16 \mathrm{H}-2,101-101$ & 141.70 & 141.71 & 141.70 & 0.75 & GB \\
\hline $16 \mathrm{H}-2,108-110$ & 141.77 & 141.79 & 141.78 & 2.00 & GB \\
\hline $16 \mathrm{H}-2,127-131$ & 141.95 & 141.99 & 141.97 & 4.00 & GB \\
\hline $16 \mathrm{H}-2,136-138$ & 142.04 & 142.06 & 142.05 & 2.00 & GB \\
\hline $16 \mathrm{H}-3,2-3$ & 142.18 & 142.19 & 142.18 & 1.00 & GB \\
\hline $16 \mathrm{H}-3,7-8$ & 142.23 & 142.24 & 142.23 & 1.00 & GB \\
\hline $16 \mathrm{H}-3,13-17$ & 142.29 & 142.33 & 142.31 & 4.00 & GB \\
\hline $16 \mathrm{H}-3,22-22$ & 142.38 & 142.39 & 142.38 & 0.75 & $\mathrm{~GB}$ \\
\hline $16 \mathrm{H}-3,49-53$ & 142.65 & 142.69 & 142.67 & 4.00 & GB \\
\hline $16 \mathrm{H}-3,74-77$ & 142.90 & 142.93 & 142.91 & 3.00 & GB \\
\hline $16 \mathrm{H}-3,78-80$ & 142.94 & 142.96 & 142.95 & 2.00 & GB \\
\hline $16 \mathrm{H}-3,110-114$ & 143.26 & 143.30 & 143.28 & 4.00 & GB \\
\hline $16 \mathrm{H}-4,36-54$ & 144.16 & 144.34 & 144.25 & 18.00 & GB \\
\hline $17 \mathrm{H}-2,75-80$ & 151.21 & 151.26 & 151.24 & 4.00 & $\mathrm{~S}$ \\
\hline $17 \mathrm{H}-2,135-142$ & 151.81 & 151.88 & 151.85 & 7.00 & $\mathrm{~GB}$ \\
\hline $17 \mathrm{H}-4,14-16$ & 153.68 & 153.70 & 153.69 & 2.00 & $\mathrm{~s}$ \\
\hline $20 \mathrm{H}-3,14-16$ & 180.48 & 180.50 & 180.49 & 2.00 & GB \\
\hline $21 \mathrm{H}-2,32-32$ & 188.24 & 188.25 & 188.24 & 0.75 & GB \\
\hline $2 \mathrm{IH}-5,75-75$ & 193.38 & 193.39 & 193.38 & 0.75 & $\mathrm{~GB}$ \\
\hline $21 \mathrm{H}-6,77-80$ & 194.90 & 194.93 & 194.91 & 3.00 & GB \\
\hline
\end{tabular}

Notes: Type $=$ lithology given in core description from Kennett, Baldauf, et al. (1994) or interpreted by this study ( $\mathrm{GB}=$ gray bed; $\mathrm{S}=$ sand). ${ }^{*}$ Event number $15(?)$ at interval $7 \mathrm{H}-4,118-135 \mathrm{~cm}$, corresponds to the magnetic susceptibility peak identified by Rack and Merrill (this volume); event numbers are used to correlate peaks between Holes $893 \mathrm{~A}$ and $893 \mathrm{~B}$.

The lack of geotechnical measurements (e.g., shear strength, consolidation tests) and the disturbed condition of the cores recovered at Site 893 (caused by gas expansion) precludes a more thorough geotechnical analysis of these cores. If earthquake-initiated mass flows can be recognized in the sediment record, it might be possible to use the magnetic susceptibility record to assess the number and frequency of these events; although changes in relative sea level and sediment accumulation rates may also be contributing factors in creating slope instability. Detailed studies of sedimentary structures across selected (thick) gray layers and massive deposits may help to determine the origins of these deposits (see Behl, this volume).

\section{Inferred Changes in Paleoclimatic Conditions}

One of the most intriguing hypotheses for explaining the series of large susceptibility peaks located at $\sim 16$ to $20 \mathrm{mbsf}(10-13 \mathrm{ka})$ is the suggestion that they are the results of a period of increased precipitation in southern California and the entire western region of North America, and that this response was caused by significant increases in continental runoff to the SBB.

Probably the best supporting evidence for this hypothesis is the observed increase in alder pollen concentrations within this specific interval of Hole 893A (see Heusser, this volume). Alder populations are generally concentrated along stream beds in the Santa BarbaraVentura region, thus providing a probable link between increased stream flow and increased flux of alder pollen to the basin (L. Heusser, pers. comm.). We have not yet been able to closely examine the other intervals of Hole 893A where alder concentrations increase, but a preliminary examination of the data suggests that associations between alder pollen and increases in magnetic susceptibility may also be observed within the following intervals: $\sim 80-90 \mathrm{ka}, \sim 105-110 \mathrm{ka}$, and $\sim 125-130 \mathrm{ka}$ (see Huesser, this volume, for information on the temporal distribution of alder pollen increases).

Observations suggesting increased mean precipitation within the interval from $\sim 10-13 \mathrm{ky}$ can frequently be found in the palynological literature concerned with the western region of North America, from California to British Columbia (Heusser, 1978; Heusser et al., 1980, 1985; Mathewes and Heusser, 1981; Rypins et al., 1989; Thompson et al., 1993). A comparison of the Hole 893A data with the climatic record from Clear Lake, California (Adams, 1988; Gardner et al., 
Table 4. Gray beds, massive beds, and sand layers in Hole 893B.

\begin{tabular}{|c|c|c|c|c|c|}
\hline \multirow{2}{*}{$\begin{array}{l}\text { Core, section, } \\
\text { interval }(\mathrm{cm})\end{array}$} & \multicolumn{3}{|c|}{ Void-corrected depth (mbsf) } & \multirow{2}{*}{$\begin{array}{l}\text { Layer } \\
\text { thickness } \\
\text { (cm) }\end{array}$} & \multirow[b]{2}{*}{ Type } \\
\hline & Top & Bottom & Midpoint & & \\
\hline \multicolumn{6}{|l|}{ 146-893B- } \\
\hline $4 \mathrm{H}-1,49-54$ & 21.79 & 21.84 & 21.82 & 5.00 & DNSC \\
\hline $4 \mathrm{H}-\mathrm{I}, 76-78$ & 22.06 & 22.08 & 22.07 & 2.00 & DNSC \\
\hline $4 \mathrm{H}-1,80-85$ & 22.10 & 22.15 & 22.13 & 5.00 & DNSC \\
\hline $4 \mathrm{H}-\mathrm{I}, 149-150$ & 22.79 & 22.80 & 22.80 & 1.00 & DNSC \\
\hline $4 \mathrm{H}-2,16-17$ & 22.96 & 22.97 & 22.97 & 1.00 & DNSC \\
\hline $4 \mathrm{H}-2,48-50$ & 23.28 & 23.30 & 23.29 & 2.00 & DNSC \\
\hline $4 \mathrm{H}-2,75-76$ & 23.55 & 23.56 & 23.56 & 1.00 & DNSC \\
\hline $4 \mathrm{H}-2,100-103$ & 23.80 & 23.83 & 23.82 & 3.00 & DNSC \\
\hline $4 \mathrm{H}-2,128-130$ & 24.04 & 24.06 & 24.05 & 2.00 & DNSC \\
\hline $4 \mathrm{H}-2,137-139$ & 24.13 & 24.15 & 24.14 & 2.00 & DNSC \\
\hline $4 \mathrm{H}-2,140-141$ & 24.16 & 24.17 & 24.17 & 1.00 & DNSC \\
\hline $4 \mathrm{H}-2,148-149$ & 24.24 & 24.25 & 24.25 & 1.00 & DNSC \\
\hline $4 \mathrm{H}-3,11-12$ & 24.36 & 24.37 & 24.37 & 1.00 & $\mathrm{~S}$ \\
\hline $4 \mathrm{H}-5,7-8$ & 27.17 & 27.18 & 27.17 & 1.00 & S \\
\hline $4 \mathrm{H}-5,9-10$ & 27.19 & 27.20 & 27.19 & 1.00 & $\mathrm{~s}$ \\
\hline $4 \mathrm{H}-5,77-78$ & 27.84 & 27.85 & 27.85 & 1.00 & s \\
\hline $4 \mathrm{H}-6,78-79$ & 29.29 & 29.30 & 29.30 & 1.00 & S \\
\hline $6 \mathrm{H}-2,10-13$ & 41.90 & 41.93 & 41.92 & 3.00 & $\mathrm{~S}$ \\
\hline $6 \mathrm{H}-3,110-121$ & 44.19 & 44.30 & 44.24 & 11.00 & S \\
\hline $6 \mathrm{H}-4,29-32$ & 44.79 & 44.82 & 44.81 & 3.00 & $\mathrm{~S}$ \\
\hline $6 \mathrm{H}-4,76-77$ & 45.26 & 45.27 & 45.26 & 1.00 & S \\
\hline $6 \mathrm{H}-5,126-127$ & 47.21 & 47.22 & 47.22 & 1.00 & S \\
\hline $6 \mathrm{H}-5,129-130$ & 47.24 & 47.25 & 47.25 & 1.00 & $\mathrm{~S}$ \\
\hline $6 \mathrm{H}-7,25-27$ & 49.14 & 49.16 & 49.15 & 2.00 & GB \\
\hline $7 \mathrm{H}-1,149-150$ & 51.29 & 51.30 & 51.29 & 1.00 & GB \\
\hline $7 \mathrm{H}-2,0-3$ & 51.29 & 51.32 & 51.31 & 3.00 & GB \\
\hline $7 \mathrm{H}-2,31-35$ & 51.56 & 51.60 & 51.58 & 4.00 & GB \\
\hline $7 \mathrm{H}-4,70-74$ & 54.72 & 54.76 & 54.74 & 4.00 & $\mathrm{~S}$ \\
\hline $7 \mathrm{H}-5,3-7$ & 55.31 & 55.35 & 55.33 & 4.00 & GB \\
\hline $7 \mathrm{H}-5,77-80$ & 56.25 & 56.28 & 56.26 & 3.00 & GB \\
\hline $7 \mathrm{H}-5,97-102$ & 56.45 & 56.50 & 56.47 & 5.00 & GB \\
\hline $7 \mathrm{H}-5,126-129$ & 56.74 & 56.77 & 56.76 & 3.00 & GB \\
\hline $7 \mathrm{H}-5,136-137$ & 56.84 & 56.85 & 56.85 & 1.00 & GB \\
\hline $7 \mathrm{H}-6,0-7$ & 56.97 & 57.04 & 57.01 & 7.00 & GB \\
\hline $7 \mathrm{H}-6,36-37$ & 57.33 & 57.34 & 57.33 & 1.00 & $\mathrm{~s}$ \\
\hline $7 \mathrm{H}-6,87-91$ & 57.84 & 57.88 & 57.86 & 4.00 & $\mathrm{~S}$ \\
\hline $8 \mathrm{H}-2,8-11$ & 60.57 & 60.60 & 60.58 & 3.00 & $\mathrm{~S}$ \\
\hline $8 \mathrm{H}-2,133-139$ & 60.93 & 60.99 & 60.96 & 6.00 & S \\
\hline $8 \mathrm{H}-3,50-52$ & 61.59 & 61.61 & 61.60 & 2.00 & $\mathrm{~S}$ \\
\hline $8 \mathrm{H}-3,127-128$ & 62.30 & 62.31 & 62.31 & 1.00 & $\mathrm{~S}$ \\
\hline $8 \mathrm{H}-6,0-6$ & 65.00 & 65.06 & 65.03 & 6.00 & S \\
\hline $8 \mathrm{H}-5,73-143$ & 65.73 & 66.43 & 66.08 & 70.00 & $\mathrm{~S}$ \\
\hline
\end{tabular}

Notes: Type = lithology given in core description from Kennett, Baldauf, et al. (1994) or interpreted by this study (DNSC = diatom nannofossil silty clay; $\mathrm{GB}=$ gray bed; $S=$ sand).

1988; Sims et al., 1988), should provide more detailed information for conducting regional comparisons of paleoclimatic variables. Studies of stream aggradation in fluvial sequences from the Antelope Valley, California, on the eastern slope of the Transverse Ranges may also provide support for increased precipitation in southern and central California from 10 to $14 \mathrm{ka}$ (Ponti, 1985).

An atmospheric mechanism for providing increased precipitation to central and southern California was suggested by the COHMAP modeling results (COHMAP Members, 1988; Kutzbach and Webb, 1993; Thompson et al., 1993). These model results suggest an increase in storm tracks across the southwest United States, which was caused by a southerly displaced westerly jet stream over North America between 15 and 9 k.y. The boundary conditions used in these simulations may need to be revised to provide more realistic model results, or to resolve shorter time-steps (Kutzbach and Ruddiman, 1993; Peltier, 1994).

\section{Comparisons With Global Records of Paleoclimate}

Additional comparisons can be attempted between the Site 893 magnetic susceptibility data and the oxygen isotope record from the Greenland Ice-core Project (GRIP) ice core from Summit, central Greenland (Dansgaard et al,, 1993; GRIP Members, 1993; Fig. 14). The data from the Summit, Greenland, location are generally comparable to the data collected from the Greenland Ice Sheet Project 2 (GISP2) ice core (Alley et al., 1993; Grootes et al., 1993; Taylor et al., 1993a, 1993b; Mayewski et al., 1994), although there may be some deformation in the lowermost portion of the record (Taylor et al., 1993a).

The two ice-core records provide the most detailed paleo-records of atmospheric variables presently available; thus, we hope that these preliminary comparisons will provide some insight into the possible atmospheric controls on the susceptibility record at Site 893 (i.e., through inferences about periods of atmospheric reorganizations and the timing of these rapid changes; Alley et al., 1993; Dansgaard et al., 1993; GRIP Members, 1993; Grootes et al., 1993; Taylor et al., 1993a, 1993b; Mayewski et al., 1994).

A recent study of past lake levels at Searles Lake in southeastern California was used to speculate that there may be a link between western North America and Greenland that is driven by oscillations in Atlantic Ocean thermohaline circulation and their influence on variations in the strength of the global hydrologic cycle (Phillips et al., 1994). Other recent studies have suggested linkages between periods of North Atlantic Deep Water production and temperature changes over Greenland (Keigwin et al., 1994) or have suggested a regionally coherent climate response between surface ocean conditions in the North Atlantic and the Greenland ice-core records (McManus et al., 1994).

\section{SUMMARY AND CONCLUSIONS}

This study has presented magnetic susceptibility measurements made on whole core, split core and discrete samples from Site 893, in the Santa Barbara Basin. These measurements were evaluated to show the general relationships between magnetic susceptibility, index properties (density, porosity, and water content), and geochemical variables (weight percentage of calcium carbonate and total organic carbon) at this site.

Magnetic susceptibility profiles, which are shown to faithfully record the presence of "gray beds" in the sediment column, were used to correlate between Holes 893A and 893B and thus transfer the Hole $893 \mathrm{~A}$ age model to Hole 893B. We propose that magnetic susceptibility fluxes can be used to provide a proxy indicator of the relative intensity of terrigenous input into the Santa Barbara Basin, and can also be used to examine changes in the geochemical environment of the basin through time. Preliminary observations suggest that magnetic susceptibility and physical properties measurements are highly sensitive to relative changes in the flux of biogenic sedimentary components and to relative changes in the accumulation of organic carbon.

Finally, comparisons were made between the magnetic susceptibility data from Hole 893A and other available paleoclimatic records. The conclusions that can be drawn from these preliminary comparisons suggest that the magnetic susceptibility record from Site 893 may provide a multichannel proxy record of paleoclimatic and paleoceanographic changes; long-term trends may correspond to fluctuations in relative sea level in the Santa Barbara Channel while higher frequency fluctuations in the detrital (terrigenous) influx into the basin may be caused by increases (decreases) in precipitation and continental runoff. Both of these signals seem to be modulated by biogeochemical changes associated with oxygen levels in bottom waters and export production of phytoplankton from surface waters.

\section{REFERENCES}

Adams, D.P., 1988. Correlations of the Clear Lake, California, core CL-73-4 pollen sequence with other long climate records. In Sims, J.D. (Ed.), Late Quaternary Climate, Tectonism, and Sedimentation in Clear Lake, Northern California Coast Ranges. Spec. Pap.-Geol. Soc. Am., 214:81-95.

Alley, R.B., Meese, D.A., Shuman, C.A., Gow, A.J., Taylor, K.C., Grootes, P.M., White, J.W.C., Ram, M., Waddington, E.D., Mayewski, P.A., and Zielinski, G.A., 1993. Abrupt increase in Greenland snow accumulation at the end of the Younger Dryas event. Nature, 362:527-529. 
Bachman, R.T.. 1984. Intratest porosity in foraminifera. J. Sediment. Petrol., $54: 257-262$

Bard, E., Fairbanks, R., Arnold, M., Maurice, P., Duprat, J., Moyes, J., and Duplessy, J.-C., 1989. Sea-level estimates during the last deglaciation based on $\delta^{18} \mathrm{O}$ and accelerator mass spectrometry ${ }^{14} \mathrm{C}$ ages measured in Globigerina bulloides. Quat. Res., 31:381-391.

Bard, E., Hamelin, B., Fairbanks, R.G., and Zinder, A., 1990. Calibration of the ${ }^{14} \mathrm{C}$ time-scale over the past 30,000 years using mass spectrometric U-Th ages from Barbados corals. Nature, 345:405-410.

Berelson, W.M., Hammond, D.E., and Johnson, K.S., 1987. Benthic fluxes and the cycling of biogenic silica and carbon in two southern California borderland basins. Geochim. Cosmochim. Acta, 51:1345-1363.

Berger, W.H., 1990. The Younger Dryas cold spell-a quest for causes. Palaeogeogr., Palaeoclimatol., Palaeoecol., 89:219-237.

Bloemendal, J., and deMenocal, P., 1989. Evidence for a change in the periodicity of tropical climate cycles at $2.4 \mathrm{Myr}$ from whole-core magnetic susceptibility measurements. Nature, 342:897-900.

Bloemendal, J., King, J.W., Hall, F.R., and Doh, S.-J., 1992. Rock magnetism of late Neogene and Pleistocene deep-sea sediments: relationship to sediment source, diagenetic processes, and sediment lithology. J. Geophys. Res., 97:4361-4375.

Bloemendal, J., King, J.W., Hunt, A., deMenocal, P.B., and Hayashida, A., 1993. Origin of the sedimentary magnetic record at Ocean Drilling Program sites on the Owen Ridge, western Arabian Sea. J. Geophys. Res., 98:4199-4219.

Bloemendal, J., Lamb, B., and King, J., 1988. Paleoenvironmental implications of rock-magnetic properties of late Quaternary sediment cores from the eastern equatorial Atlantic. Paleoceanography, 3:61-87.

Booth, J.S., and Dahl, A.G., 1986. A note on the relationships between organic matter and some geotechnical properties of a marine sediment. Mar, Geotechnol., 6:281-297.

Brandsma, D., Lund, S.P., and Henyey, T.L., 1989. Paleomagnetism of Late Quaternary marine sediments from Santa Catalina basin, California continental borderland. J. Geophys. Res., 94:547-564.

Brownlie, W.R., and Taylor, B.D., 1981. Sediment management for Southern California mountains, coastal plains and shoreline. Calif. Inst. of Technol., Envir. Quality Lab. Rep., 17-C.

Busch, W.H., and Keller, G.H., 1982. Consolidation characteristics of sediments from the Peru-Chile Continental Margin and implications for past sediment instability. Mar. Geol., 45:17-39.

- 1983. Analysis of sediment stability on the Peru-Chile Continental Slope. Mar. Geotechnol., 5:181-211.

Charles, C.D., Rind, D., Jouzel, J., Koster, R.D., and Fairbanks, R.G., 1994 Glacial-interglacial changes in moisture sources for Greenland: influences on the ice core record of climate. Science, 263:508-511.

Cooperative Holocene Mapping Project (COHMAP) Members, 1988. Climatic changes of the last 18,000 years: observations and model simulations. Science, 241:1043-1052.

Dansgaard, W., Johnsen, S.J., Clausen, H.B., Dahl-Jensen, D., Gundstrup, N.S., Hammer, C.U., Hvidberg, C.S., Steffensen, J.P., Sveinbjörnsdottir, A.E., Jouzel, J., and Bond, G., 1993. Evidence for general instability of past climate from a 250-kyr ice-core record. Nature, 364:218-220.

deMenocal, P., Bloemendal, J., and King, J., 1991. A rock-magnetic record of monsoonal dust deposition to the Arabian Sea: evidence for a shift in the mode of deposition at $2.4 \mathrm{Ma}$. In Prell, W.L., Niitsuma, N., et al., Proc. ODP, Sci. Results, 117: College Station, TX (Ocean Drilling Program), 389-407.

Doh, S.-J., King, J.W., and Leinen, M., 1988. A rock-magnetic study of giant piston core LL4-GPC3 from the central North Pacific and its paleoceanographic implications. Paleoceanography, 3:89-111.

Donnellan, A., Hager, B.H., King, R.W., and Herring. T.A., 1993. Geodetic measurements of deformation in the Ventura Basin region, southern California. J. Geophys. Res., 98:21727-21739.

Drake, D.E., Kolpack, R.L., and Fischer, P.J., 1972. Sediment transport on the Santa Barbara-Oxnard shelf, Santa Barbara Channel, California. In Swift, D.J.P., Duane, D.B., and Pilkey, O.H. (Eds.), Shelf Sediment Transport: Stroudsburg, PA (Dowden, Hutchinson, and Ross), 307-331.

Edwards, R.L., Beck, J.W., Burr, G.S., Donahue, D.J., Chappell, J.M.A. Bloom, A.L., Druffel, E.R.M., and Taylor, F.W., 1993. A large drop in atmospheric ${ }^{14} \mathrm{C} /{ }^{12} \mathrm{C}$ and reduced melting in the Younger Dryas, documented with ${ }^{230} \mathrm{Th}$ ages of coral. Science, 260:962-968.

Emery, K.O., and Hülsemann, J., 1962. The relationships of sediments, life and water in a marine basin. Deep-Sea Res. Part A, 8:165-180.
Fairbanks, R.G., 1989. A 17,000-year glacio-eustatic sea level record: influence of glacial melting rates on the Younger Dryas event and deep-ocean circulation. Nature, 342:637-642.

Field, M.E., and Edwards, B.D., 1980. Slopes of the southern California Continental Borderland: a regime of mass transport. In Field, M.E. Bouma, A.H., Colburn, I.P., Douglas, R.G., and Ingle, J.C. (Eds.), Quaternary Depositional Environments of the Pacific Coast. Pacific Sect. Soc. Econ. Paleontol. Mineral., 169-184.

Fleischer, P., 1972. Mineralogy and sedimentation history, Santa Barbara Basin, California. J. Sediment. Petrol., 42:49-58.

Gallup, C.D., Edwards, R.L., and Johnson, R.G., 1994. The timing of high sea levels over the past 200,000 years. Science, 263:796-800.

Gardner, J.V., Heusser, L.E., Quinterno, P.J., Stone, S.M., Barron, J.A., and Poore, R.Z., 1988. Clear Lake record vs. the adjacent marine record: a correlation of their past 20,000 years of paleoclimatic and paleoceanographic responses. In Sims, J.D. (Ed.), Late Quaternary Climate, Tectonism, and Sedimentation in Clear Lake, Northern California Coast Ranges. Spec. Pap.-Geol. Soc. Am., 214:171-182.

Gorsline, D.S., Kolpack, R.L., Karl, H.A., Drake, D.E., Fleischer, P., Thornton, S.E., Schwalbach, J.R., and Savrda, C.E., 1984. Studies of finegrained sediment transport processes and products in the California Continental Borderland. In Stow, D.A.V., and Piper, D.J.W. (Eds.), FineGrained Sediments: Deep-water Processes and Facies. Geol. Soc. Spec. Publ. London, 15:375-415.

Greenland Ice-core Project (GRIP) Members, 1993. Climate instability during the last interglacial period recorded in the GRIP ice core. Nature, 364:203-207.

Grootes, P.M., Stuvier, M., White, J.W.C., Johnsen, S., and Jouzel, J., 1993. Comparison of oxygen isotope records from the GISP2 and GRIP Greenland ice cores. Nature, 366:552-554.

Grousset, F.E., Labeyrie, L., Sinko, J.A., Cremer, M., Bond, G., Duprat, J., Cortijo, E., and Huon, S., 1993. Patterns of ice-rafted detritus in the glacial North Atlantic $\left(40-55^{\circ} \mathrm{N}\right)$. Paleoceanography, 8:175-192.

Hamilton, E.L., 1976. Variations of density and porosity with depth in deepsea sediments. J. Sediment. Petrol., 46:280-300.

Heusser, C.J., Heusser, L.E., and Peteet, D.M., 1985. Late Quaternary climatic change on the American North Pacific coast. Nature, 315:485-487.

Heusser, C.J., Heusser, L.E., and Streeter, S.S., 1980. Quaternary temperatures and precipitation for the north-west coast of North America. Nature, 286:702-704.

Heusser, L.E., 1978. Marine pollen in Santa Barbara Basin, California: a 12,000-yr record. Geol. Soc. Am. Bull., 89:673-678.

Imbrie, J., Hays, J.D., Martinson, D.G., McIntyre, A., Mix, A.C., Morley, J.J., Pisias, N.G., Prell, W.L., and Shackleton, N.J., 1984. The orbital theory of Pleistocene climate: support from a revised chronology of the marine $\delta^{18} \mathrm{O}$ record. In Berger, A., Imbrie, J., Hays, J., Kukla, G., and Saltzman, B. (Eds.), Milankovitch and Climate (Pt. 1): Dordrecht (D. Reidel), 269-305.

Jahnke, R.A., 1990. Early diagenesis and recycling of biogenic debris at the seafloor, Santa Monica Basin, California. J. Mar. Res., 48:413-436.

Karlin, R., 1990a. Magnetic diagenesis in marine sediments from the Oregon continental margin. J. Geophys. Res., 95:4405-4419.

1990b. Magnetite mineral diagenesis in suboxic sediments at Bettis Site W-N, NE Pacific Ocean. J. Geophys. Res., 95:4421-4436.

Karlin, R., and Levi, S., 1983. Diagenesis of magnetic minerals in recent hemipelagic sediments. Nature, 303:327-330.

-1985. Geochemical and sedimentological control of the magnetic properties of hemipelagic sediments. J. Geophys. Res., 90:10373-10392.

Keigwin, L.D., Curry, W.B., Lehman, S.J., and Johnsen, S., 1994. The role of the deep ocean in North Atlantic climate change between 70 and 130 kyr ago. Nature, 371:323-326.

Keller, G.H., 1982. Organic matter and the geotechnical properties of submarine sediments. Geo-Mar. Lett., 2:191-198.

Kennett, J.P., Baldauf, J.G., et al., 1994. Proc. ODP, Init. Repts., 146 (Pt. 2): College Station, TX (Ocean Drilling Program).

Kolpack, R.L., 1986. Sedimentology of the mainland nearshore region of Santa Barbara Channel, California. In Knight, R.J., and McLean, J.R. (Eds.), Shelf Sands and Sandstones. Mem._Can. Soc. Pet. Geol., 11:5772.

Kolpack, R.L., and Drake, D.E., 1985. Transport of clays in the eastern part of Santa Barbara Channel, California. Geo-Mar. Lett., 4:191-196.

Kutzbach, J.E., and Ruddiman, W.F., 1993. Model description, external forcing, and surface boundary conditions. In Wright, H.E., Jr., Kutzbach, J.E., Webb, T., Jr., Ruddiman, W.F., Street-Perrott, F.A., and Bartlein, 
P.J. (Eds.), Global Climates Since the Last Glacial Maximum: Minneapolis (Univ. Minnesota Press), 12-23.

Kutzbach, J.E., and Webb, T., III, 1993. Conceptual basis for understanding Late-Quaternary climates. In Wright, H.E., Jr., Kutzbach, J.E., Webb, T. Jr., Ruddiman, W.F., Street-Perrott, F.A., and Bartlein, P.J. (Eds.), Global Climates Since the Last Glacial Maximum: Minneapolis (Univ. Minnesota Press), 5-11.

Lange, C.B., Berger, W.H., Burke, S.K., Casey, R.E., Schimmelmann, A., Soutar, A., and Weinheimer, A.L., 1987. El Niño in Santa Barbara basin: diatom, radiolarian, and foraminiferan responses to the "1983 El Niño" event. Mar. Geol., 78:153-160.

Lange, C.B., Burke, S.K., and Berger, W.H., 1990. Biological production off southern California is linked to climatic change. Clim. Change, 16:319329.

Larson, K.M., 1993. Application of the Global Positioning System to crustal deformation measurements, 3. Results from the southern California Borderlands. J. Geophys. Res., 98:21713-21726.

Larson, K.M., and Webb, F.H., 1992. Deformation in the Santa Barbara Channel from GPS measurements 1987-1991. Geophys. Res. Lett., 19:1491-1494

Lee, H.J., 1982. Bulk density and shear strength of several deep-sea calcareous sediments. In Demars, K.R., and Chaney, R.C. (Eds.), Geotechnical Properties, Behavior, and Performance of Calcareous Soils. ASTM Spec. Tech. Publ., 777:54-78.

Lee, H.J., and Edwards, B.D., 1986. Regional method to assess offshore slope stability. J. Geotech. Div., Am. Soc. Civ. Eng., 112:489-509.

Lee, W.H.K., and Vedder, J.G., 1973. Recent earthquake activity in the Santa Barbara Channel region. Bull. Seis. Soc. Am., 63:1757-1773.

Leslie, B.W., Hammond, D.E., Berelson, W.M., and Lund, S.P., 1990a. Diagenesis in anoxic sediments from the California Continental Borderland and its influence on iron, sulfur, and magnetite behavior. J. Geophys. Res., 95:4453-4470.

Leslie, B.W., Lund, S.P., and Hammond, D.E., 1990b. Rock magnetic evidence for the dissolution and authigenic growth of magnetic minerals within anoxic marine sediments of the California Continental Borderland. J. Geophys. Res., 95:4437-4452.

Lund, S.P., Gorsline, D.L., and Henyey, T.L., 1992. Rock magnetic characteristics of surficial sediments from the California continental borderland. Earth Planet. Sci. Lett., 108:93-107.

Maher, B.A., and Thompson, R., 1991. Mineral magnetic record of the Chinese loess and paleosols. Geology, 19:3-6.

Mathewes, R.W., and Heusser, L.E., 1981. A 12,000 year palynological record of temperature and precipitation trends in southwestern British Columbia. Can. J. Bot., 59:707-710.

Mayer, L.A., 1979. Deep sea carbonates: acoustic, physical, and stratigraphic properties. J. Sediment. Petrol., 49:819-836.

Mayewski, P.A., Meeker, L.D., Whitlow, S., Twickler, M.S., Morrison, M.C., Bloomfield, P., Bond, G.C., Alley, R.B., Gow, A.J., Grootes, P.M., Meese, D.A., Ram, M., Taylor, K.C., and Wumkes, W., 1994. Changes in atmospheric circulation and ocean ice cover over the North Atlantic during the last 41,000 years. Science, 263:1747-1751.

McManus, J.F., Bond, G.C., Broecker, W.S., Johnsen, S., Labeyrie, L., and Higgins, S., 1994. High-resolution climate records from the North Atlantic during the last interglacial. Nature, 371:326-329.

Peltier, W.R., 1994. Ice age paleotopography. Science, 265:195-201.

Phillips, F.M., Campbell, A.R., Smith, G.I., and Boschoff, J.L., 1994. Interstadial climatic cycles: a link between western North America and Greenland? Geology, 22:1115-1118.

Ponti, D.J., 1985. The Quaternary alluvial sequence of the Antelope Valley, California. In Weide, D.L., and Faber, M.L. (Eds.), Soils and Quaternary Geology of the Southwestern United States. Spec. Pap.-Geol. Soc. Am., 203:79-96

Prior, D.B., and Coleman, J.M., 1984. Submarine slope instability. In Brunsden, D., and Prior, D.B. (Eds.), Slope Instability: New York (Wiley), 419-455.

Pusch, R., 1973. Influence of organic matter on the geotechnical properties of clays. Natl. Swed. Bldg. Res., 11.

Reimers, C.E., Lange, C.B., Tabak, M., and Bernhard, J.M., 1990. Seasonal spillover and varve formation in the Santa Barbara Basin, California. Limnol. Oceanogr., 35:1577-1585.

Reynolds, S., 1987. A recent turbidity current event, Hueneme Fan, California: reconstruction of flow properties. Sedimentology, 34:129-137.
Robinson, S.G., 1986. The late Pleistocene paleoclimatic record of North Atlantic deep-sea sediments revealed by mineral-magnetic measurements. Phys. Earth Planet. Inter., 42:22-47.

Rypins, S., Reneau, S.L., Byrne, R., and Montgomery, D.R., 1989. Palynologic and geomorphic evidence for environmental change during the Pleistocene-Holocene transition at Point Reyes Peninsula, central coastal California. Quat. Res., 32:72-87.

Saltzman, B., and Verbitsky, M., 1994. $\mathrm{CO}_{2}$ and glacial cycles. Nature, $367: 419$.

Schimmelmann, A., and Kastner, M., 1993. Evolutionary changes over the last 1000 years of reduced sulfur phases and organic carbon in varved sediments of the Santa Barbara Basin. California. Geochim. Cosmochim. Acta, 57:67-78.

Schimmelmann, A., Lange, C.B., and Berger, W.H., 1990. Climatically controlled marker layers in Santa Barbara basin sediments, and fine-scale core-to-core correlation. Limnol. Oceanogr., 35:165-173.

Schimmelmann, A., Lange, C.B., Berger, W.H., Simon, A., Burke, S.K., and Dunbar, R.B., 1992. Extreme climatic conditions recorded in Santa Barbara Basin laminated sediments: the 1835-1840 Macoma event. Mar. Geol., 106:279-299.

Schwalbach, J.R., and Gorsline, D.S., 1985. Holocene sediment budgets for the basins of the California Continental Borderland. J. Sediment. Petrol., $55: 829-842$.

Shaw, J.H., and Suppe, J., 1994. Active faulting and growth faulting in the eastern Santa Barbara Channel, California. Geol. Soc. Am. Bull., 106:607-626.

Sims, J.D., Rymer, M.J., and Perkins, J.A., 1988. Late Quaternary deposits beneath Clear Lake, California: physical stratigraphy, age, and paleogeographic implications. In Sims, J.D. (Ed.), Late Quaternary Climate, Tectonism, and Sedimentation in Clear Lake, Northern California Coast Ranges. Spec. Pap.-Geol. Soc. Am., 214:21-44.

Soutar, A., and Crill, P.A., 1977. Sedimentation and climatic patterns in the Santa Barbara Basin during the 19th and 20th centuries. Geol. Soc. Am. Bull., 88:1161-1172.

Stanley, D.J., and Warne, A.G., 1994. Worldwide initiation of Holocene marine deltas by deceleration of sea-level rise. Science, 265:228-231.

Taylor, K.C., Hammer, C.U., Alley, R.B., Clausen, H.B., Dahl-Jensen, D., Gow, A.J., Gundestrup, N.S., Kipfstuhl, J., Moore, J.C., and Waddington, E.D., 1993. Electrical conductivity measurements from the GISP2 and GRIP Greenland ice cores. Nature, 366:549-552.

Taylor, K.C., Lamorey, G.W., Doyle, G.A., Alley, R.B., Grootes, P.M., Mayewski, P.A., White, J.W.C., and Barlow, L.K., 1993. The "flickering switch" of late Pleistocene climate change. Nature, 361:432-436.

Thompson, R., and Oldfield, F., 1986. Environmental Magnetism: London (Allen and Unwin).

Thompson, R.S., Whitlock, C., Bartlein, P.J., Harrison, S.P., and Spaulding, W.G., 1993. Climatic changes in the western United States since 18,000 yr B.P. In Wright, H.E., Jr., Kutzbach, J.E., Webb, T., Jr., Ruddiman, W.F., Street-Perrott, F.A., and Bartlein, P.J. (Eds.), Global Climates Since the Last Glacial Maximum: Minneapolis (Univ. Minn. Press), 468513.

Thornton, S.E., 1981. Suspended sediment transport in surface waters of the California Current off southern California: 1977-78 floods. Geo-Mar. Lett., 1:23-28.

1984. Basin model for hemipelagic sedimentation in a tectonically active continental margin: Santa Barbara Basin, California continental borderland. In Stow, D.A.V., and Piper, D.J.W. (Eds.), Finegrained Sediments: Deep-water Processes and Facies. Geol. Soc, Spec. Publ. London, 15:377-394.

, 1986. Origin of mass flow sedimentary structures in hemipelagic basin deposits: Santa Barbara basin. California Borderland. Geo-Mar. Lett., 6:15-19.

Tushingham, A.M., and Peltier, W.R., 1991. ICE-3G: a new global model of late Pleistocene deglaciation based upon geophysical predictions of postglacial relative sea level change. J. Geophys. Res., 96:4497-4523.

Yeats, R.S., 1983. Large-scale Quaternary detachments in Ventura Basin, southern California. J. Geophys. Res., 88:569-583.

Date of initial receipt: 1 September 1994

Date of acceptance: 7 March 1995

Ms 146SR-284 


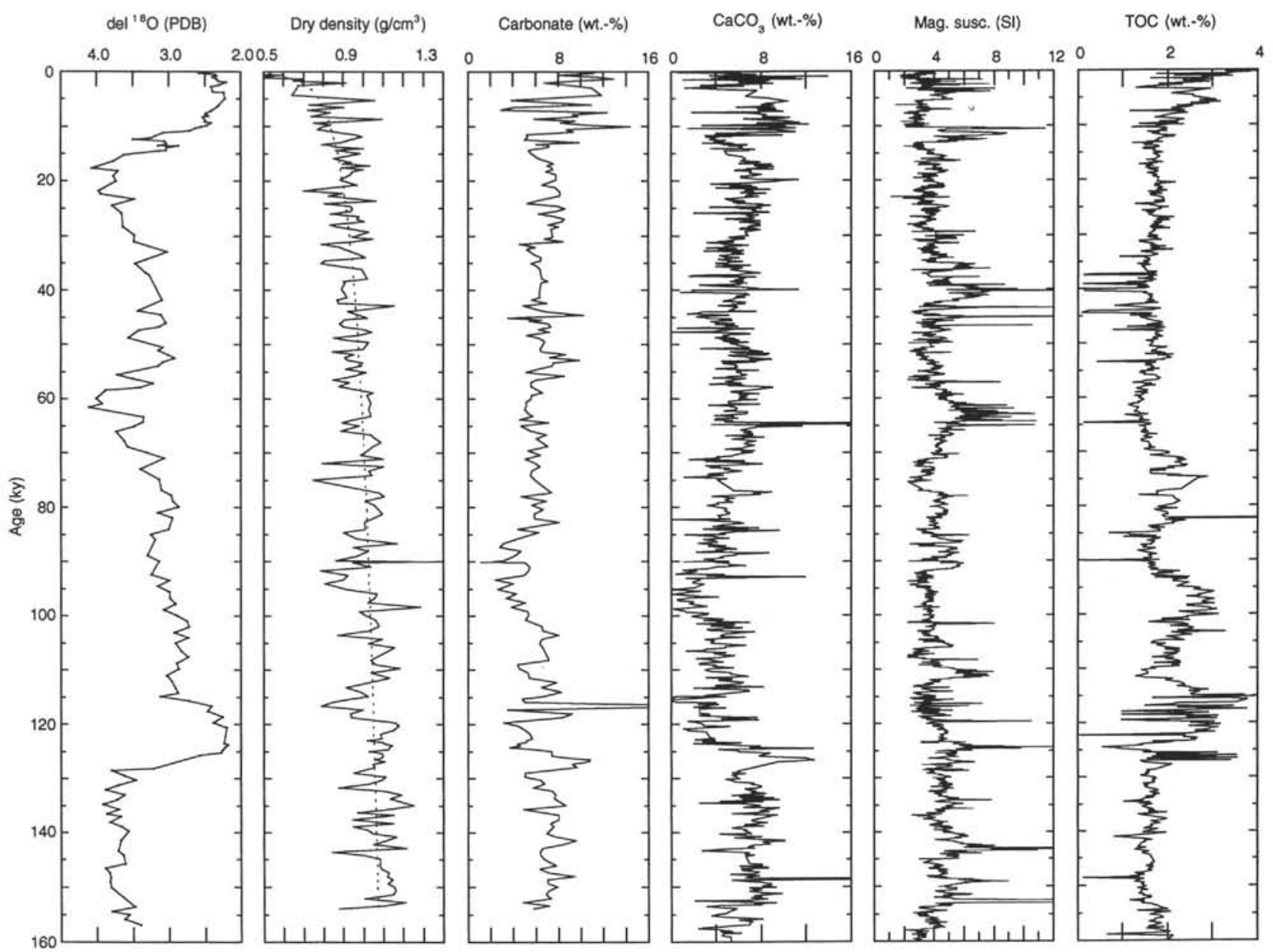

Figure 11. Comparisons between magnetic susceptibility measurements and laboratory determinations of the weight percentage of calcium carbonate and total organic carbon (Stein and Rack, this volume) using samples from Hole 893A. Discrete determinations of dry density and the weight percentage of calcium carbonate (this paper) and the oxygen isotope stratigraphy of Kennett (this volume) are also plotted vs. age (in k.y.). 


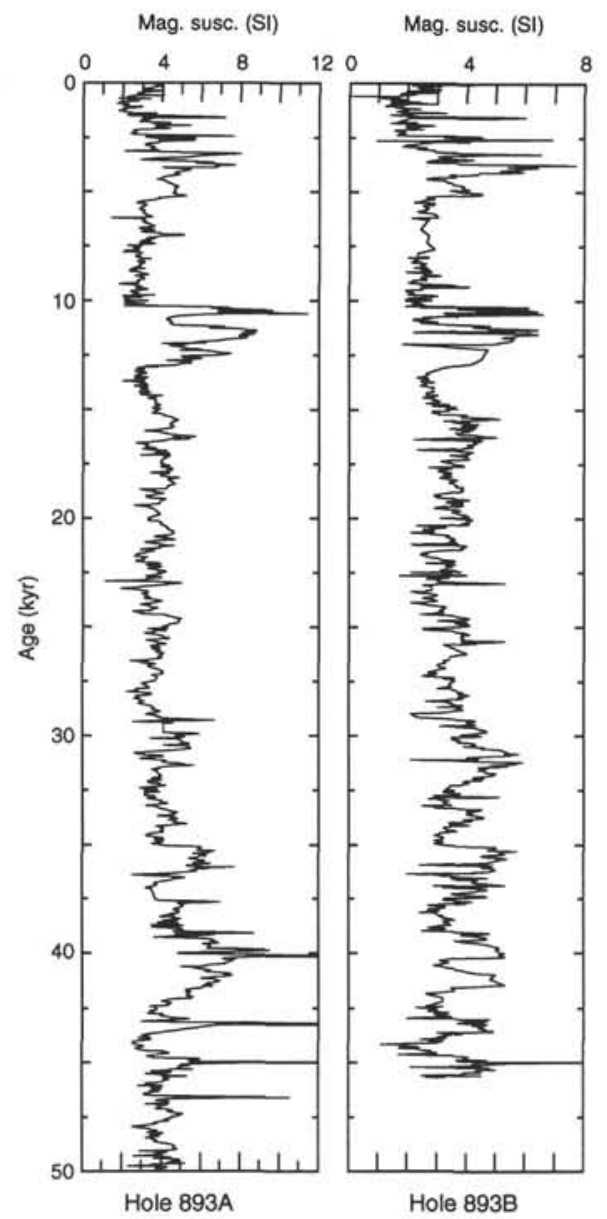

Figure 12. This figure shows the correlation between magnetic susceptibility profiles from Holes $893 \mathrm{~A}$ and $893 \mathrm{~B}$, plotted vs. age (k.y.). These correlations are based on 39 tie-points listed in Table 5; the correlation coefficient for the match between these two records is 0.614 .
Table 5. Correlations between Holes 893A and 893B using "AnalySeries" software and Hole 893A age model.*

\begin{tabular}{|c|c|c|c|}
\hline $\begin{array}{l}\text { Hole 893B } \\
\text { depth** } \\
\text { (mbsf) }\end{array}$ & $\begin{array}{l}\text { Age } \\
\text { (ka) }\end{array}$ & $\begin{array}{l}\text { Hole 893A } \\
\text { depth** } \\
\text { (mbsf) }\end{array}$ & $\begin{array}{l}\text { Difference } \dagger \\
\text { (m) }\end{array}$ \\
\hline 0.00 & 0.000 & 0.00 & 0.00 \\
\hline 0.70 & 0.069 & 0.15 & 0.55 \\
\hline 2.22 & & & $\mathrm{IH} / 2 \mathrm{H}(\mathrm{B})$ \\
\hline 4.04 & 1.269 & 2.75 & 1.29 \\
\hline 4.54 & 1.453 & 3.15 & 1.39 \\
\hline 5.14 & 1.740 & 3.75 & 1.39 \\
\hline 5,82 & 2.147 & 4.50 & 1.32 \\
\hline 6.27 & 2.336 & 4.85 & 1.42 \\
\hline 7.08 & 2.742 & 5.60 & 1.48 \\
\hline \multirow[t]{2}{*}{7.72} & 3.184 & 6.03 & 1.69 \\
\hline & & 6.50 & $1 \mathrm{H} / 2 \mathrm{H}(\mathrm{A})$ \\
\hline 9.87 & 4.418 & 7.13 & 2.74 \\
\hline 10.77 & 5.450 & 8.05 & 2,72 \\
\hline 11.37 & 6.123 & 8.65 & 2.72 \\
\hline 11.62 & 6.370 & 9.01 & 2.61 \\
\hline 11.80 & & & $2 \mathrm{H} / 3 \mathrm{H}$ (B) \\
\hline 11.87 & 7.933 & 11.62 & 0.25 \\
\hline 13.88 & 9.142 & 13.64 & 0.24 \\
\hline 14.77 & 9.626 & 14.62 & 0.15 \\
\hline \multirow[t]{2}{*}{15.27} & 9.912 & 15.27 & 0.00 \\
\hline & & 16.00 & $2 \mathrm{H} / 3 \mathrm{H}(\mathrm{A})$ \\
\hline 16.49 & 10.238 & 16.01 & 0.48 \\
\hline 17.84 & 10.256 & 16.05 & 1.79 \\
\hline 18.98 & 10.844 & 17.17 & 1.81 \\
\hline 20.61 & 11.974 & 18.97 & 1.64 \\
\hline 20.86 & 13.052 & 20.66 & 0.20 \\
\hline \multirow[t]{2}{*}{21.30} & & & $3 \mathrm{H} / 4 \mathrm{H}$ (B) \\
\hline & & 25.50 & $3 \mathrm{H} / 4 \mathrm{H}(\mathrm{A})$ \\
\hline 27.06 & 16.282 & 25.61 & 1.45 \\
\hline 30.07 & 18.972 & 30.15 & -0.08 \\
\hline 30.83 & & & $4 \mathrm{H} / 5 \mathrm{H}(\mathrm{B})$ \\
\hline 33.99 & 21.689 & 32.81 & 1.18 \\
\hline \multirow[t]{2}{*}{35.95} & 22.907 & 34.56 & 1.39 \\
\hline & & 35.00 & $4 \mathrm{H} / 5 \mathrm{H}(\mathrm{A})$ \\
\hline 40.30 & & & $5 \mathrm{H} / 6 \mathrm{H}$ (B) \\
\hline \multirow[t]{2}{*}{44.12} & 29.004 & 43.32 & 0.80 \\
\hline & & 44.48 & $5 \mathrm{H} / 6 \mathrm{H}(\mathrm{A})$ \\
\hline 44.85 & 30.141 & 44.90 & -0.05 \\
\hline 46.80 & 31.522 & 46.82 & -0.02 \\
\hline 49.69 & 33.192 & 49.14 & 0.55 \\
\hline 49.80 & & & $6 \mathrm{H} / 7 \mathrm{H}(\mathrm{B})$ \\
\hline 51.80 & 35.055 & 51.73 & 0.07 \\
\hline \multirow[t]{2}{*}{53.25} & 36.322 & 53.49 & -0.24 \\
\hline & & 54.05 & $6 \mathrm{H} / 7 \mathrm{H}(\mathrm{A})$ \\
\hline 54.92 & 37.523 & 55.16 & -0.24 \\
\hline 57.42 & 38.977 & 57.18 & 0.24 \\
\hline 59.14 & 40.293 & 59.01 & 0.13 \\
\hline 59.29 & 40.610 & 59.45 & -0.16 \\
\hline 59.30 & & & $7 \mathrm{H} / 8 \mathrm{H}(\mathrm{B})$ \\
\hline \multirow[t]{2}{*}{59.61} & 42.402 & 61.94 & -2.33 \\
\hline & & 63.65 & $7 \mathrm{H} / 8 \mathrm{H}(\mathrm{A})$ \\
\hline 65.79 & 44.927 & 65.45 & 0.34 \\
\hline 67.59 & 45.683 & 66.50 & 1.09 \\
\hline
\end{tabular}

Notes: ${ }^{*}$ See Table 2. **Depths are void-corrected. $†$ Difference $=893 \mathrm{~B}-893 \mathrm{~A}$. See Appendixes $\mathrm{A}$ and $\mathrm{B}$ (on CD-ROM accompanying this volume) for magnetic susceptibility values corresponding to void-corrected depths. The depth at the top of each core is shown for each hole, for example, $1 \mathrm{H} / 2 \mathrm{H}(\mathrm{A})=$ top of Core $146-893 \mathrm{~A}$ $2 \mathrm{H}$. For the two magnetic susceptibility records correlated here, the resulting correlation coefficient $=0.614$. 


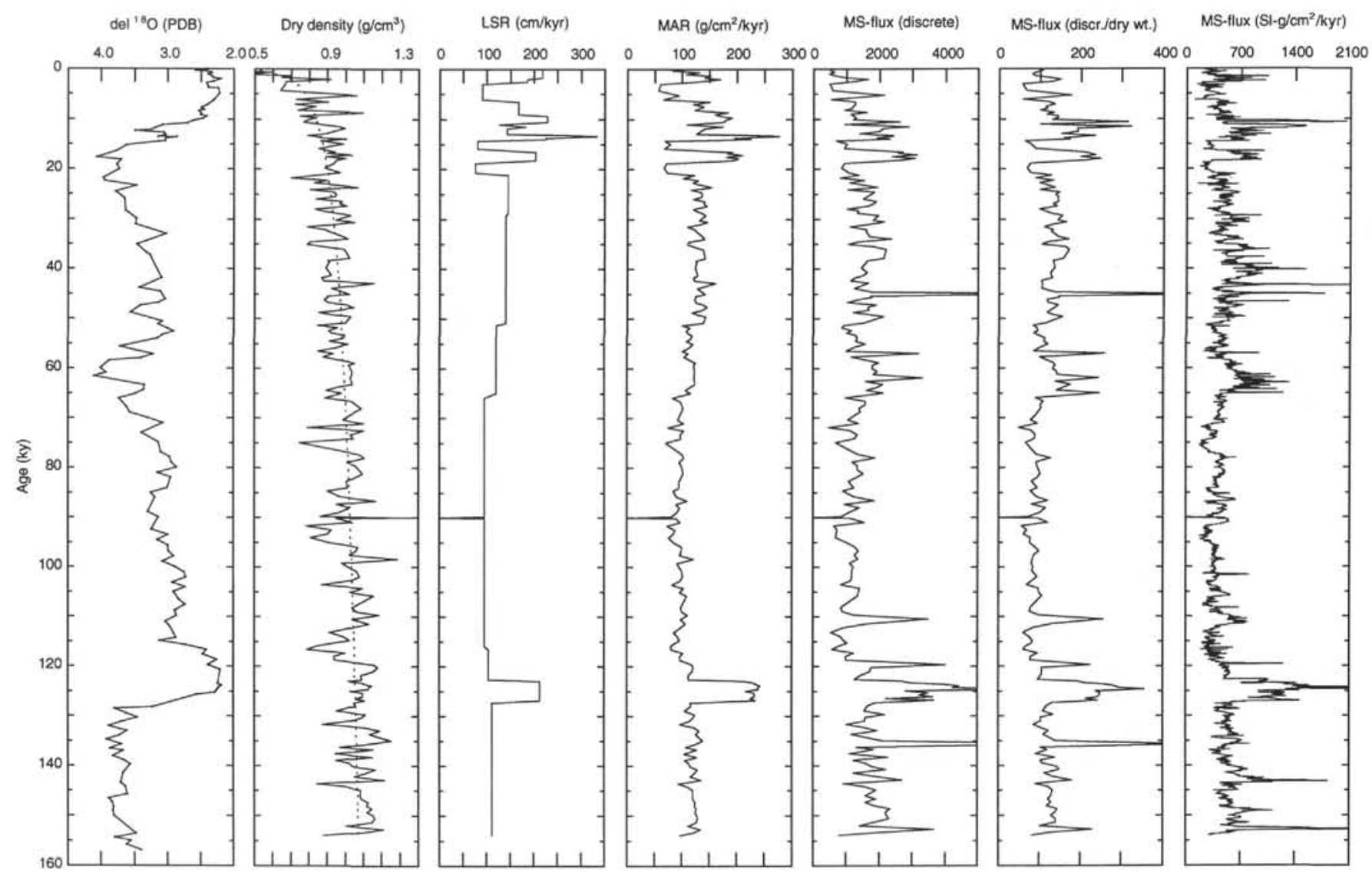

Figure 13. Summary of magnetic susceptibility flux calculations for Hole 893A; the data are plotted vs. age (k.y.). LSR = linear sedimentation rate, MAR = mass accumulation rate, MS-flux = magnetic susceptibility flux calculated for discrete samples, discrete values normalized by dry sample weight, and split-core measurements, respectively. The oxygen isotope stratigraphy of Kennett (this volume) is also shown. 


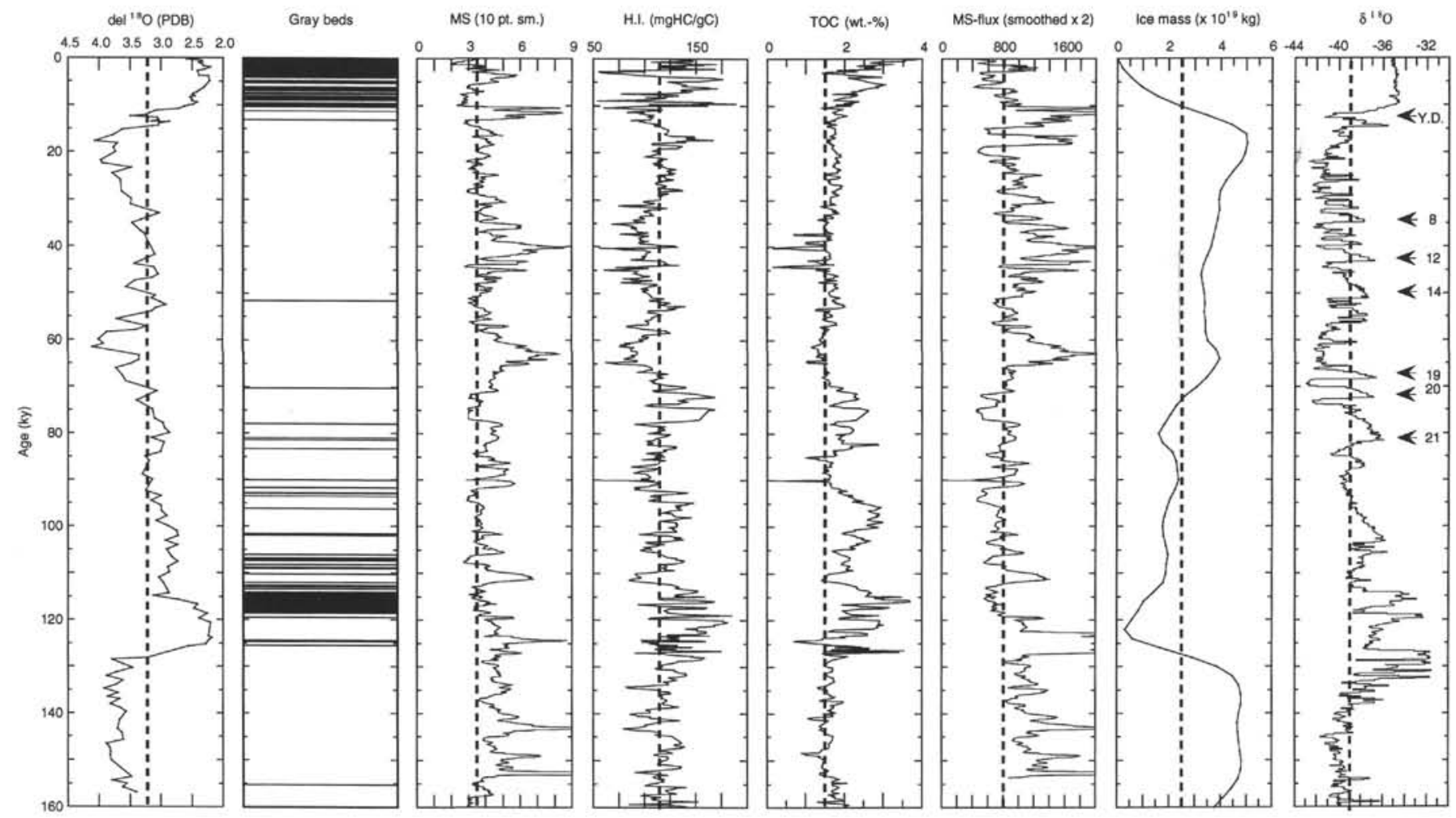

Figure 14. Comparisons between split-core magnetic susceptibility measurements, magnetic susceptibility flux (10-point smooth), total organic carbon, and the hydrogen index (Stein and Rack, this volume). Also shown are (1) oxygen isotope stratigraphy of Kennett (this volume); (2) the positions of gray beds in Hole $893 \mathrm{~A}$; (3) ice mass deduced from the SPECMAP oxygen isotope stack (Imbrie et al., 1984); and (4) $\delta^{18} \mathrm{O}$ determinations from the GRIP ice core. The GRIP $\delta^{18} \mathrm{O}$ curve has been annotated to identify the Younger Dryas (YD) period and selected interstadials (after Dansgaard et al., 1993). 
APPENDIX A

Magnetic Susceptibility Measurements, Hole 893A

\begin{tabular}{|c|c|c|c|}
\hline $\begin{array}{l}\text { Core, section, } \\
\text { interval }(\mathrm{cm})\end{array}$ & $\begin{array}{l}\text { Void-corrected } \\
\text { depth } \\
\text { (mbsf) }\end{array}$ & $\begin{array}{l}\text { Age } \\
\text { (ka) }\end{array}$ & $\begin{array}{c}\text { Magnetic } \\
\text { susceptibility } \\
\text { (SI units) }\end{array}$ \\
\hline \multicolumn{4}{|l|}{$146-893 \mathrm{~A}-$} \\
\hline $\mathrm{H}-1,0$ & 0.00 & 0.000 & 2.10 \\
\hline IH-1,5 & 0.05 & 0.023 & 3.90 \\
\hline $1 \mathrm{H}-1,10$ & 0.10 & 0.046 & 3.60 \\
\hline $1 \mathrm{H}-1,15$ & 0.15 & 0.069 & 3.70 \\
\hline $1 \mathrm{H}-1,20$ & 0.20 & 0.092 & 3.70 \\
\hline $1 \mathrm{H}-1,25$ & 0.25 & 0.115 & 3.80 \\
\hline $\mathrm{IH}-1,30$ & 0.30 & 0.138 & 3.90 \\
\hline IH-1, 35 & 0.35 & 0.162 & 3.70 \\
\hline $1 \mathrm{H}-1,40$ & 0.40 & 0.184 & 3.50 \\
\hline $1 \mathrm{H}-1.45$ & 0.45 & 0.208 & 3.40 \\
\hline $\mathrm{IH}-\mathrm{I}, 50$ & 0.50 & 0.231 & 3.30 \\
\hline IH-1, 55 & 0.55 & 0.254 & 3.00 \\
\hline $\mathrm{IH}-1.60$ & 0.60 & 0.277 & 3.00 \\
\hline $\mathrm{IH}-1,65$ & 0.65 & 0.300 & 3.10 \\
\hline IH-1. 70 & 0.70 & 0.323 & 3.00 \\
\hline IH-1.75 & 0.75 & 0.346 & 4.00 \\
\hline $\mathrm{IH}-1,80$ & 0.80 & 0.369 & 3.70 \\
\hline $\mathrm{IH}-1,85$ & 0.85 & 0.392 & 3.50 \\
\hline $\mathrm{IH}-\mathrm{I} .90$ & 0.90 & 0.415 & 2.90 \\
\hline IH-1.95 & 0.95 & 0.438 & 2.70 \\
\hline IH-1, 100 & 1.00 & 0.461 & 2.80 \\
\hline IH-I. 105 & 1.05 & 0.484 & 2.90 \\
\hline $1 \mathrm{H}-1,110$ & 1.10 & 0.507 & 2.80 \\
\hline $1 \mathrm{H}-1,115$ & 1.15 & 0.530 & 3.00 \\
\hline $1 \mathrm{H}-1.120$ & 1.20 & 0.554 & 4.10 \\
\hline IH-1, 125 & 1.25 & 0.577 & 2.70 \\
\hline $\mathrm{IH}-1,130$ & 1.30 & 0.600 & 2.20 \\
\hline $1 \mathrm{H}-1.135$ & 1.35 & 0.623 & 2.40 \\
\hline $1 \mathrm{H}-1,140$ & 1.40 & 0.646 & 2.60 \\
\hline $1 \mathrm{H}-1.145$ & 1.45 & 0.669 & 2.30 \\
\hline $\mathrm{IH}-1.150$ & 1.50 & 0.692 & 1.80 \\
\hline $\mathrm{IH}-2,0$ & 1.50 & 0.692 & 2.00 \\
\hline $\mathrm{IH}-2,5$ & 1.55 & 0.715 & 3.40 \\
\hline $\mathrm{IH}-2,10$ & 1.60 & 0.738 & 3.00 \\
\hline $1 \mathrm{H}-2,15$ & 1.65 & 0.761 & 2.20 \\
\hline IH- 2,20 & 1.70 & 0.784 & 2.70 \\
\hline $\mathrm{IH}-2,25$ & 1.75 & 0.807 & 2.80 \\
\hline $\mathrm{IH}-2,30$ & 1.80 & 0.830 & 2.30 \\
\hline $\mathrm{IH}-2,35$ & 1.85 & 0.854 & 2.00 \\
\hline $\mathrm{IH}-2,40$ & 1.90 & 0.877 & 1.80 \\
\hline $1 \mathrm{H}-2,45$ & 1.95 & 0.900 & 1.90 \\
\hline $1 \mathrm{H}-2,50$ & 2.00 & 0.923 & 1.70 \\
\hline $\mathrm{IH}-2,55$ & 2.05 & 0.946 & 2.20 \\
\hline IH-2. 60 & 2.10 & 0.969 & 2.30 \\
\hline $\mathrm{IH}-2,65$ & 2.15 & 0.992 & 2.00 \\
\hline $1 \mathrm{H}-2,70$ & 2.20 & 1.015 & 2.10 \\
\hline $1 \mathrm{H}-2,75$ & 2.25 & 1.038 & 2.30 \\
\hline $1 \mathrm{H}-2,80$ & 2.30 & 1.061 & 2.20 \\
\hline $1 \mathrm{H}-2,85$ & 2.35 & 1.084 & 3.00 \\
\hline $\mathrm{IH}-2,90$ & 2.40 & 1.107 & 2.70 \\
\hline IH-2, 95 & 2.45 & 1.130 & 2.00 \\
\hline $1 \mathrm{H}-2,100$ & 2.50 & 1.153 & 2.00 \\
\hline $\mathrm{IH}-2,105$ & 2.55 & 1.176 & 2.10 \\
\hline $\mathrm{IH}-2,110$ & 2.60 & 1.199 & 2.20 \\
\hline $1 \mathrm{H}-2,115$ & 2.65 & 1.222 & 1.90 \\
\hline $1 \mathrm{H}-2,120$ & 2.70 & 1.246 & 1.80 \\
\hline $1 \mathrm{H}-2,125$ & 2.75 & 1.269 & 2.00 \\
\hline $\mathrm{IH}-2,130$ & 2.80 & 1.292 & 2.10 \\
\hline $1 \mathrm{H}-2,135$ & 2.85 & 1.315 & 2.00 \\
\hline $1 \mathrm{H}-2,140$ & 2.90 & 1.338 & 2.40 \\
\hline $1 \mathrm{H}-2,145$ & 2.95 & 1.361 & 2.50 \\
\hline $1 \mathrm{H}-2,150$ & 3.00 & 1.384 & 3.70 \\
\hline IH-3,5 & 3.05 & 1.407 & 2.50 \\
\hline $\mathrm{IH}-3, \mathrm{IO}$ & 3.10 & 1.430 & 2.30 \\
\hline $1 \mathrm{H}-3,15$ & 3.15 & 1.453 & 2.20 \\
\hline $1 \mathrm{H}-3,20$ & 3.20 & 1.476 & 2.50 \\
\hline IH-3.25 & 3.25 & 1.499 & 3.90 \\
\hline $1 \mathrm{H}-3,30$ & 3.30 & 1.522 & 6.70 \\
\hline $\mathrm{IH}-3,35$ & 3.35 & 1.545 & 7.20 \\
\hline $1 \mathrm{H}-3,40$ & 3,40 & 1.569 & 3.80 \\
\hline $1 \mathrm{H}-3,45$ & 3.45 & 1.592 & 3.00 \\
\hline IH-3, 50 & 3.50 & 1.615 & 3.40 \\
\hline IH-3,55 & 3.55 & 1.638 & 3.30 \\
\hline IH-3, 60 & 3.60 & 1.661 & 3.10 \\
\hline $1 \mathrm{H}-3,65$ & 3.65 & 1.686 & 2.90 \\
\hline $\mathrm{IH}-3,70$ & 3.70 & 1.713 & 3.00 \\
\hline $1 \mathrm{H}-3,75$ & 3.75 & 1.740 & 2.80 \\
\hline $1 \mathrm{H}-3.80$ & 3.80 & 1.768 & 3.10 \\
\hline $1 \mathrm{H}-3,85$ & 3.85 & 1.794 & 4.40 \\
\hline $1 \mathrm{H}-3,90$ & 3.90 & 1.822 & 3.50 \\
\hline $1 \mathrm{H}-3,95$ & 3.95 & 1.849 & 3,70 \\
\hline $\mathrm{IH}-3,100$ & 4.00 & 1.876 & 3.70 \\
\hline
\end{tabular}

APPENDIX B

Magnetic Susceptibility Measurements, Hole 893B

\begin{tabular}{|c|c|c|c|}
\hline $\begin{array}{l}\text { Core, section, } \\
\text { interval }(\mathrm{cm})\end{array}$ & $\begin{array}{l}\text { Void-corrected } \\
\text { depth (mbsf) }\end{array}$ & Age (ka) & $\begin{array}{c}\text { Magnetic } \\
\text { susceptibility } \\
\text { (SI units) }\end{array}$ \\
\hline \multicolumn{4}{|l|}{ 146-893B- } \\
\hline $\begin{array}{l}\mathrm{IH}-\mathrm{I}, 0 \\
\mathrm{IH}-\mathrm{I}, 5\end{array}$ & $\begin{array}{l}0.00 \\
0.05\end{array}$ & $\begin{array}{l}0.000 \\
0.005\end{array}$ & $\begin{array}{l}1.80 \\
4.40\end{array}$ \\
\hline IH-1, 10 & 0.10 & 0.010 & 3.80 \\
\hline $1 \mathrm{H}-1,15$ & 0.15 & 0.015 & 4.20 \\
\hline $1 \mathrm{H}-1,20$ & 0.20 & 0.020 & 4.20 \\
\hline IH-1,25 & 0.25 & 0.025 & 3.70 \\
\hline $\mathrm{IH}-1,30$ & 0.30 & 0.030 & 3.10 \\
\hline IH-I, 35 & 0.35 & 0.034 & 3.10 \\
\hline $1 \mathrm{H}-1,40$ & 0.40 & 0.039 & 3.10 \\
\hline $\mathrm{IH}-1,45$ & 0.45 & 0.044 & 3.20 \\
\hline $\mathrm{IH}-1,50$ & 0.50 & 0.049 & 2.90 \\
\hline $1 \mathrm{H}-1,55$ & 0.55 & 0.054 & 2.70 \\
\hline $\mathrm{IH}-\mathrm{I}, 60$ & 0.60 & 0.059 & 2.20 \\
\hline $1 \mathrm{H}-1,65$ & 0.65 & 0.064 & 2.10 \\
\hline $1 \mathrm{H}-1.75$ & 0.70 & 0.069 & 2.20 \\
\hline $1 \mathrm{H}-1,80$ & 0.75 & 0.087 & 3.10 \\
\hline IH-1, 85 & 0.80 & 0.105 & 3.10 \\
\hline $1 \mathrm{H}-1,90$ & 0.85 & 0.123 & 2.90 \\
\hline IH-I, 95 & 0.90 & 0.141 & 2.70 \\
\hline $\mathrm{IH}-\mathrm{I}, 100$ & 0.95 & 0.159 & 2.50 \\
\hline $1 \mathrm{H}-1.105$ & 1.00 & 0.177 & 2.30 \\
\hline $1 \mathrm{H}-\mathrm{I}, 110$ & 1.05 & 0.195 & 2.50 \\
\hline $1 \mathrm{H}-1,115$ & 1.10 & 0.213 & 2.60 \\
\hline $1 \mathrm{H}-1,120$ & 1.15 & 0.231 & 2.50 \\
\hline $\mathrm{IH}-1,125$ & 1.20 & 0.249 & 3.00 \\
\hline $1 \mathrm{H}-1,130$ & 1.25 & 0.267 & 3.10 \\
\hline IH-2, 5 & 1.48 & 0.349 & 1.90 \\
\hline $1 \mathrm{H}-2,10$ & 1.53 & 0.367 & 2.20 \\
\hline $1 \mathrm{H}-2.15$ & 1.58 & 0.385 & 3.00 \\
\hline $\mathrm{IH}-2,20$ & 1.63 & 0.403 & 2.80 \\
\hline IH $-2,25$ & 1.68 & 0.421 & 1.90 \\
\hline $\mathrm{IH}-2,30$ & 1.73 & 0.439 & 1.70 \\
\hline $\mathrm{IH}-2,35$ & 1.78 & 0.457 & 2.00 \\
\hline IH-2, 40 & 1.83 & 0.475 & 2.30 \\
\hline IH-2. 45 & 1.88 & 0.493 & 2.10 \\
\hline $1 \mathrm{H}-2,50$ & 1.93 & 0.511 & 1.80 \\
\hline $\mathrm{IH}-2,55$ & 1.98 & 0.529 & 1.70 \\
\hline $\mathrm{IH}-2.60$ & 2.03 & 0.547 & 1.70 \\
\hline IH-CC, 0 & 2.07 & 0.561 & 0.00 \\
\hline IH-CC, 5 & 2.12 & 0.579 & 1.10 \\
\hline IH-CC, 10 & 2.16 & 0.594 & 1.20 \\
\hline IH-CC. 15 & 2.21 & 0.612 & 1.60 \\
\hline $2 \mathrm{H}-1,10$ & 2.32 & 0.651 & 1.00 \\
\hline $2 \mathrm{H}-1,15$ & 2.37 & 0.669 & 1.40 \\
\hline $2 \mathrm{H}-1,20$ & 2.42 & 0.687 & 1.80 \\
\hline $2 \mathrm{H}-1,25$ & 2.47 & 0.705 & 1.90 \\
\hline $2 \mathrm{H}-\mathrm{I}, 30$ & 2.52 & 0.723 & 2.00 \\
\hline $2 \mathrm{H}-1,35$ & 2.57 & 0.741 & 1.90 \\
\hline $2 \mathrm{H}-\mathrm{I}, 40$ & 2.62 & 0.759 & 1.60 \\
\hline $2 \mathrm{H}-1.45$ & 2.67 & 0.777 & 1.40 \\
\hline $2 \mathrm{H}-1,50$ & 2.72 & 0.795 & 1.50 \\
\hline $2 \mathrm{H}-1,55$ & 2.77 & 0.813 & 2.40 \\
\hline $2 \mathrm{H}-1,60$ & 2.82 & 0.831 & 2.20 \\
\hline $2 \mathrm{H}-1.65$ & 2.87 & 0.849 & 1.60 \\
\hline $2 \mathrm{H}-1,70$ & 2.92 & 0.867 & 1.60 \\
\hline $2 \mathrm{H}-1,75$ & 2.97 & 0.885 & 1.40 \\
\hline $2 \mathrm{H}-\mathrm{I}, 80$ & 3.02 & 0.903 & 3.00 \\
\hline $2 \mathrm{H}-1.85$ & 3,07 & 0.920 & 2.10 \\
\hline $2 \mathrm{H}-1.90$ & 3.12 & 0.938 & 1.70 \\
\hline $2 \mathrm{H}-1,95$ & 3.17 & 0.956 & 1.50 \\
\hline $2 \mathrm{H}-1.100$ & 3.22 & 0.974 & 1.30 \\
\hline $2 \mathrm{H}-1,105$ & 3.27 & 0.992 & 1.30 \\
\hline $2 \mathrm{H}-1,110$ & 3.32 & 1.010 & 1.20 \\
\hline $2 \mathrm{H}-1,115$ & 3.37 & 1.028 & 1.50 \\
\hline $2 \mathrm{H}-1,120$ & 3.42 & 1.046 & 1.60 \\
\hline $2 \mathrm{H}-1,125$ & 3.47 & 1.064 & 1.60 \\
\hline $2 \mathrm{H}-1,130$ & 3.52 & 1.082 & 1.70 \\
\hline $2 \mathrm{H}-1,135$ & 3.57 & 1.100 & 1.90 \\
\hline $2 \mathrm{H}-1,140$ & 3.62 & 1.118 & 1.70 \\
\hline $2 \mathrm{H}-1,145$ & 3.67 & 1.136 & 2.40 \\
\hline $2 \mathrm{H}-1.150$ & 3.72 & 1.154 & 1.80 \\
\hline $2 \mathrm{H}-2,5$ & 3.79 & 1.179 & 1.90 \\
\hline $2 \mathrm{H}-2,10$ & 3.84 & 1.197 & 1.80 \\
\hline $2 \mathrm{H}-2,15$ & 3.89 & 1.215 & 1.80 \\
\hline $2 \mathrm{H}-2,20$ & 3.94 & 1.233 & 2.00 \\
\hline $2 \mathrm{H}-2,25$ & 3.99 & 1.251 & 1.60 \\
\hline $2 \mathrm{H}-2,30$ & 4.04 & 1.269 & 1.70 \\
\hline $2 \mathrm{H}-2,35$ & 4.09 & 1.287 & 1.80 \\
\hline $2 \mathrm{H}-2,40$ & 4.14 & 1.306 & 2.30 \\
\hline $2 \mathrm{H}-2,45$ & 4.19 & 1.324 & 3.30 \\
\hline $2 \mathrm{H}-2,50$ & 4.24 & 1.343 & 2.20 \\
\hline $2 \mathrm{H}-2,55$ & 4.29 & 1.361 & 2.10 \\
\hline
\end{tabular}

Only part of these tables are reproduced here. The entire appendixes appear on the CD-ROM (back pocket). 